\title{
Um estudo das hipersuperfícies maximais tipo espaço no espaço anti-de Sitter
}

\author{
Bruno Mascaro \\ DisSERTAÇÃO APRESENTADA \\ $\mathrm{AO}$ \\ Instituto de Matemática e Estatística \\ DA \\ UNIVERSIDADE DE SÃo PAUlo \\ PARA \\ OBTENÇÃO DO TÍTULO \\ DE \\ Mestre em Matemática \\ Programa: Mestrado em Matemática \\ Orientador: Prof ${ }^{\mathrm{a}}$. Dr ${ }^{\mathrm{a}}$. Barbara Corominas Valério
}

Durante o desenvolvimento deste trabalho o autor recebeu auxílio financeiro da CNPq

São Paulo, Junho de 2017 


\section{Um estudo das hipersuperfícies maximais tipo espaço no espaço anti-de Sitter}

\footnotetext{
Esta versão da dissertação/tese contém as correções e alterações sugeridas pela Comissão Julgadora durante a defesa da versão original do trabalho, realizada em 07/06/2017. Uma cópia da versão original está disponível no Instituto de Matemática e Estatística da Universidade de São Paulo.
}

Comissão Julgadora:

- Prof ${ }^{\mathrm{a}}$. Dr ${ }^{\mathrm{a}}$. Barbara Corominas Valério (orientadora) - IME-USP

- Prof. Dr. Luiz Amancio Machado de Sousa Junior - UNIRIO

- Prof. Dr. Juan Fernando Zapata Zapata - EEL-USP 


\section{Agradecimentos}

Gostaria de demonstrar meu profundo agradecimento a muitas pessoas, mas não há como não começar agradecendo a minha família, ao meu pai e minha mãe que sempre me proveram de todo apoio e toda paciência com as minhas decisões. Sou eternamente grato e dedico a maior parte deste trabalho à vocês, que me ajudam diariamente na busca pelos meus sonhos.

Gostaria de agradecer aos meus amigos do Insituto de Matemática e Estatística, pela companhia, pelas horas intermináveis de estudos, pelas longas conversas que tivémos durante esses anos juntos, seja sobre a matemática ou sobre qualquer assunto, sempre me ajudando com dúvidas ou sugestões, seja nesse trabalho ou em qualquer assunto que conversávamos, sou muito grato por vocês terem feito parte desse processo.

Agradeço também a minha orientadora, a professora Bárbara, que desde o meu início na graduação foi um exemplo a ser seguido, seja com as broncas ou com os conselhos dados ao longo dos anos, sempre disponível para dúvidas e se tornando uma amiga. Obrigado professora, a senhora me ajudou muito com o trabalho.

Por último mas não menos importante, gostaria de agradecer aos meus amigos dos tempos de escola, pelos finais de semana alegres e viagens divertidas.

Seria impossível deixar um agradecimento para todas as pessoas que fizeram parte deste trabalho, direta ou indiretamente, mas agradeço desde já, pois todos vocês tem uma parte fundamental na elaboração deste trabalho. 


\section{Resumo}

MASCARO, B. Um estudo das hipersuperfícies maximais tipo espaço no espaço-anti de Sitter. 2017. 71 f. Dissertação (Mestrado) - Instituto de Matemática e Estatística, Universidade de São Paulo, São Paulo, 2017.

Este trabalho apresenta a demonstração de dois teoremas sobre a caracterização de hipersuperfícies maximais no espaço anti-de Sitter. Ambos os Teoremas 4.0.1 e 4.0.2 caracterizam hipersuperfícies maximais isométricamente imersas no espaço anti-de Sitter $\mathbb{H}_{1}^{n+1}$ com (n-1) curvaturas principais de mesmo sinal, com curvatura escalar constante e curvatura de Gauss-Kronecker constante não-nula, respectivamente, como sendo isométricas ao cilindro hiperbólico $\mathbb{H}^{1}\left(c_{1}\right) \times \mathbb{H}^{n-1}\left(c_{2}\right)$. Também é feito um breve estudo do artigo [17], onde o Teorema 3.0.3 é ferramenta chave para a obtenção dos resultados demonstrados nos Teoremas 4.0.1 e 4.0.2.

Palavras-chave: hipersuperfícies maximais, espaço anti-de Sitter, cilindro hiperbólico. 


\section{Abstract}

MASCARO, B. A study of spacelike maximal hypersurfaces in the anti-de Sitter space. 2010. 120 f. Dissertação (Mestrado) - Instituto de Matemática e Estatística, Universidade de São Paulo, São Paulo, 2017.

This work presents, the demonstration of two theorems about the characterization of maximal hypersurfaces on the anti-de Sitter space. Both Theorems 4.0.1 and 4.0.2 characterize maximal hypersurfaces isometrically immersed in the anti-de Sitter space $\mathbb{H}_{1}^{n+1}$ with (n-1) principal curvatures with the same sign, with constant scalar curvature and nonzero constant Gauss-Kronecker curvature, respectively, as being isometric to the hyperbolic cylinder $\mathbb{H}^{1}\left(c_{1}\right) \times \mathbb{H}^{n-1}\left(c_{2}\right)$. Is also done a brief study of the article [17], where the Theorem 3.0.3 is key piece to obtain the results demonstrated in Theorems 4.0.1 and 4.0.2.

Keywords: maximal hypersurfaces, anti-de Sitter space, hyperbolic cylinder. 


\section{Sumário}

1 Definições $\quad 3$

1.1 Motivação histórica . . . . . . . . . . . . . . . . . . . . . . . . 3

1.2 Variedades topológicas e diferenciáveis . . . . . . . . . . . . . . . . . . . . . . 4

1.3 Curvaturas ... . . . . . . . . . . . . . . . . . . . . . . 6

1.4 Os espaços $L_{p}^{n+p}$ e a segunda forma fundamental $I I_{\eta}$. . . . . . . . . . . . . . . . . . . 7

1.4.1 A segunda forma fundamental e suas consequências . . . . . . . . . . . . . 8

1.5 Distribuições e formas diferencias . . . . . . . . . . . . . . . . . . . . . . . . 10

2 Preliminares 13

2.1 O referencial móvel em $\mathbb{H}_{1}^{n+1}$. . . . . . . . . . . . . . . . . . . . . . . . . 13

2.2 Segunda forma fundamental e a Equação De Gauss no método do referencial móvel . 17

2.3 Componentes de $\nabla h$ e a equação de Codazzi . . . . . . . . . . . . . . . . . . . . . . 18

2.4 Curvatura de Ricci e Curvatura Escalar . . . . . . . . . . . . . . . . . . . . . 23

2.5 Ferramentas técnicas ............................. 24

3 Subvariedades tipo espaço maximais em um espaço pseudo-riemanniano de curvatura constante

4 Uma caracterização de cilindros hiperbólicos 43

Referências Bibliográficas $\quad 66$

$\begin{array}{ll}\text { Índice Remissivo } & 65\end{array}$ 
viii SUMÁRIO 


\section{Introdução}

\section{O problema de Bernstein}

O problema de Bernstein é uma questão famosa na área de geometria diferencial, e originalmente discutia a questão de que se, dado um gráfico de uma função em $\mathbb{R}^{n-1}$ que é uma superfície mínima em $\mathbb{R}^{n}$, isto implicaria que a função é linear? Sergei Natanovich Bernstein, que deu nome ao problema, o resolveu para o caso $n=3 \mathrm{em} 1914$. Hoje se é sabido que a resposta é afirmativa até $n=8$, mas falsa para $n \geq 9$. Um exemplo específico de superfície que demonstra a falsidade da afirmação é $S=\left\{x \in \mathbb{R}^{8}: x_{1}^{2}+x_{2}^{2}+x_{3}^{2}+x_{4}^{2}=x_{5}^{2}+x_{6}^{2}+x_{7}^{2}+x_{8}^{2}\right\}$. Para mais informações sobre tais superfícies e exemplos, ver [3].

$\mathrm{Na}$ direção do problema de Berstein, foram propostas outras formulações e resultados análogos foram obtidos para outros ambientes e formas espaciais. Um resultado muito importante foi obtido por Calabi [6], que deu origem ao Teorema de Calabi-Bernstein.

Com esse problema foram observadas as chamadas propriedades do tipo Bernstein, que são aquelas que decidem sobre a unicidade de certos objetos matemáticos, isto é, se um objeto $X$ possuir uma propriedade específica, qualquer outro objeto $Y$, que também possua a propriedade, será necessariamente similar a $X$.

Além do interesse do ponto de vista da física no avanço da teoria, com o passar do tempo, o interesse em problemas do tipo Bernstein tem crescido bastante, e visto que, dentro do espaço Lorentziano superfícies tipo espaço com curvatura média constante, em particular as superfícies maximais, são objetos matemáticos que apresentam interessantes propriedades do tipo Bernstein, diversos estudos vêm sendo desenvolvidos neste direção.

Ao longo dos anos, diversos trabalhos que avançam nesse sentido vêm sendo publicados, dentre os quais podemos citar os de Chern, do Carmo e Kobayashi [10], Ishihara [17] e Cheng e Yau [9], como resultados famosos.

Quando o ambiente é o espaço de Sitter $\left(\mathbb{S}_{p}^{n+p}\right)$, Goddard em [15] conjecturou que toda hipersuperfície tipo espaço completa com curvatura media constante não nula em $\mathbb{S}_{1}^{n+1}$ teria que ser totalmente umbílica. Foi demonstrada a falsidade de tal conjectura em seu enunciado original, mas ela deu origem a diversos trabalhos em busca de sua demonstração, como em [1], Akutagawa mostrou que a conjectura de Goddard é verdadeira quando o quadrado da curvatura média for menor ou igual a 1 , ou seja, $H^{2} \leq 1$, para o caso $n=2$, e para $n=3$, a conjectura também é verdadeira quando $H^{2}<\frac{4(n-1)}{n^{2}}$. Para mais informações ver [19] e [4].

No caso do ambiente anti-de Sitter $\left(\mathbb{H}_{p}^{n+p}\right)$, Cao e Wei [7] mostraram que, se $n \geq 3$ então toda hipersuperficie $n$-dimensional tipo espaço completa e maximal em $\mathbb{H}_{1}^{n+1}$, com exatamente duas curvaturas principais distintas $\lambda$ e $\mu$ em todo lugar, tais que $\inf (\lambda-\mu)^{2}>0$, é isométrica ao cilindro hiperbólico $\mathbb{H}^{m}\left(-\frac{n}{m}\right) \times \mathbb{H}^{n-m}\left(-\frac{n}{n-m}\right) \quad(1 \leq m \leq n-1)$. Ao fim do artigo, uma conjectura é deixada para próximos trabalhos, afirmando que, as únicas hipersuperfícies tipo espaço no espaço anti- 
de Sitter,com curvatura média constante e duas curvaturas principais distintas $\lambda$ e $\mu$, satisfazendo $\inf (\lambda-\mu)^{2}$, são os cilindros hiperbólicos. Foi demonstrada a veracidade de tal conjectura por Wu em [26]. Em [24], pode-se ver novos exemplos de hipersuperfícies maximais e completas para o caso onde o espaço ambiente é o $\mathbb{H}_{1}^{3}$. As variedades produto, possuindo um espaço hiperbólico $\mathbb{H}^{m}\left(c_{1}\right)$ como uma das variedades, são chamados de cilindros hiperbólicos, e os resultados enunciados acima, são umas das principais caracterizações que inspiraram os resultados para novas caracterizações destes espaços.

O capítulo 1 apresenta algumas definições e resultados básicos para o desenvolvimento deste trabalho, de maneira bem geral, somente para a fixação de algumas notações e uniformização da nomenclatura aqui usada. Tais resultados são comunmente vistos em um curso de geometria Riemanniana.

No capítulo 2 é feito a dedução de algumas relações e fórmulas usadas no decorrer do trabalho, onde o método do referencial móvel em $\mathbb{H}_{1}^{n+1}$ é desenvolvido e como as fórmulas conhecidas da geometria Riemanniana ficam usando o método do referencial móvel e a linguagem de formas diferenciáveis. Alguns resultados mais técnicos também são desenvolvidos ao final deste capítulo.

Já no capítulo 3 é feito um estudo um pouco mais detalhado do artigo [17] de T. Ishihara, artigo base para a obtenção dos resultados principais de [8], onde é demonstrado que as únicas subvariedades conexas, completas, maximais tipo espaço de dimensão $n$ em $\mathbb{H}_{p}^{n+p}(-1)$ são as subvariedades $\mathbb{H}^{n_{1}}\left(\sqrt{\frac{n_{1}}{n}}\right) \times \cdots \times \mathbb{H}^{n_{p+1}}\left(\sqrt{\frac{n_{p+1}}{n}}\right)$. Também são demonstrados dois teoremas anteriores ao resultado principal e o desenvolvimento da equação tipo Simons-Calabi, que é peça chave na demonstração dos resultados contidos em [17].

Por fim, no capítulo 4, é feito o estudo do artigo de Chaves, Sousa e Valério [8], onde estuda-se as hipersuperfícies tipo espaço completas e maximais em $\mathbb{H}_{1}^{n+1}$, com curvatura escalar constante ou curvatura de Gauss-Kronecker constante não-nula, caracterizando assim os cilindros hiperbólicos como sendo as únicas tais hipersuperfícies com $(n-1)$ curvaturas principais com o mesmo sinal em toda hipersuperfície. Para a obtenção de tal resultado, faz - se o forte uso do Teorema (1.3) de [17] e o principio do maximo generalizado, encontrado em [27].

De maneira resumida, a estratégia em [8] é analisar a expressão do laplaciano da função definida em $M^{n}$

$$
F=\log \left|\operatorname{det}\left(h_{i j}\right)\right|,
$$

utilizando o método do referencial móvel, alguns lemas técnicos intermediários e resultados preliminares desenvolvidos neste trabalho, e assim, usando que a curvatura de Ricci é limitada inferiormente, juntamente com o Lema 3.0.4 os autores conseguem concluir que, sob as hipóteses, em ambos os teoremas, $S=n$ e como já foi mencionado, usam o resultado de [17], e assim, as hipersuperfícies estudadas são isométricas ao cilindro hiperbólico $\mathbb{H}^{1}\left(c_{1}\right) \times \mathbb{H}^{n-1}\left(c_{2}\right)$, e até o conhecimento dos autores, os corolários enunciados são os primeiros resultados a cerca da classificação de hipersuperfícies tipo espaço completas e maximais de $\mathbb{H}_{1}^{5}$, com curvatura escalar constante ou curvatura de Gauss-Kronecker contante. 


\section{Capítulo 1}

\section{Definições}

\subsection{Motivação histórica}

Uma das áreas mais antigas da matemática é a geometria, palavra que tem etimologia grega e tem seu significado relacionado à "medição de terras". Como o próprio significado já diz, o estudo da geometria começa com uma apelação física, sendo assim, o início do pensamento geométrico nada tinha de parecido com o que conhecemos hoje por geometria diferencial. O registro do mais antigo grego a tentar extrair o pensamento relacionado ao mundo físico para o âmbito puramente mental das idéis da geometria, foi o mercador e engenheiro Tales de Mileto ( $\pm 624 a . C . - \pm 547$ a.C.). A obra mais célebre que começa a tratar a geometria como forma de pensamento abstrato e puramente no contexto mental, é o livro Os Elementos de Euclides, composto por 13 livros (capítulos), e tão célebre que o único rival em número de traduções é a Bíblia.

Na obra de Euclides, são postulados 5 axiomas da geometria plana, onde o mais famoso sem dúvida é o quinto postulado, devido a duvida que persistiu por anos em torno dele, onde muitos tentaram e até acreditaram ter provado que este não era um axioma, e sim um teorema dos outros 4 , mas após tantas discussões, viu-se que realmente tal postulado era necessário, e o primeiro questionamento foi sobre o que aconteceria com a geometria se o quinto postulado não fosse verdade, e com isso, nasceu a primeira idéia do que conhecemos por hoje como geometria hiperbólica. Para mais informações, vide [2].

Já a geometria diferencial tem sua origem na miscigenação do clássico cálculo diferencial e integral com a geometria, onde diferentemente da geometria estudada no passado, conhecida como geometria clássica ou estática, a geometria diferencial estuda a variação infinitesimal de entidades geométricas, conhecidas de maneira geral como superfícies, e tentando quantificar certas características dos objetos estudados.

As propriedades estudadas na geometria diferencial podem ser separadas em dois grandes grupos, propriedades intrínsecas e propriedades extrínsecas.

Propriedades extrínsecas, são as que dependem do ambiente onde sua entidade geométrica está inserido, por exemplo, o caso mais natural é o $\mathbb{R}^{3}$, onde é possível enxergar e desenhar as entidades geométricas em estudo, e propriedades como o vetor normal, ou caracterizações que decorram de o objeto estar "dentro"do $\mathbb{R}^{3}$, são ditas extrínsecas.

As propriedades ditas intrínsecas são as que não dependem do ambiente, ou seja, que seja lá qual for o ambiente onde sua entidade está sendo estudado, ela tem alguma propriedade ou característica que independe disso. 
Com essas idéias, muitas perguntas foram surgindo, e matemáticos começaram a se perguntar o que aconteceria se o espaço ambiente não fosse o $\mathbb{R}^{3}$, então foram surgindo outros tipos de espaços e com isso, muitas outras perguntas, que continuam surgindo e ainda sem respostas.

\section{$1.2 \quad$ Variedades topológicas e diferenciáveis}

Muitas generalizações existem nos dias de hoje, mas todas provém da idéia central de variedade, que surge como uma alternativa aos espaços ambientes convencionais, com poucas propriedades e com o mínimo para se fazer algum tipo de geometria.

Definição 1.2.1 (Variedade topológica). Seja $M$ um espaço toplógico, diremos que $M$ é uma variedade topológica se:

- Para todo ponto $x, y \in M$, existe um par de abertos $U, V \subset M$, tais que $x \in U, y \in V$ e $U \cap V=\emptyset$;

- Existe uma base de abertos para a topologia de $M$ que é enumerável;

- Todo ponto $x \in M$ tem uma vizinhança que é homeomorfa a um aberto do $\mathbb{R}^{n}$

$O$ último item nos permite dizer que, localmente, $M$ é um espaço Euclideano, dado que ambos são espaços topológicos, e homeomorfismo conserva topologia.

Contudo, há uma classe de variedades com propriedadeds mais interessantes do ponto de vista desse trabalho, que são chamadas as variedades diferenciáveis, que ocorrem quando uma variedade topológica possui também uma estrutura diferenciável nela, a grosso modo, os homeomorfismos variam diferenciavelmente entre si, como vemos a seguir:

Definição 1.2.2 (Variedade diferenciável). Uma variedade diferenciável de dimensão $n$ é uma variedade topológica $M$ e uma familia de aplicações $\phi_{\alpha}: U_{\alpha} \subset \mathbb{R}^{n} \rightarrow M$ de abertos $U_{\alpha}$ de $\mathbb{R}^{n}$ em $M$ tais que:

- $\bigcup_{\alpha} \phi_{\alpha}\left(U_{\alpha}\right)=M$;

- Para todo par $\alpha, \beta$ com $\phi_{\alpha}\left(U_{\alpha}\right) \cap \phi_{\beta}\left(U_{\beta}\right)=W \neq \emptyset$, os conjuntos $\phi_{\alpha}^{-1}(W), \phi_{\beta}^{-1}(W)$ são abertos em $\mathbb{R}^{n}$ e as aplicações $\phi_{\beta}^{-1} \circ \phi_{\alpha}$ são diferenciáveis, dizemos então que a mudança de coordenadas é uma aplicação diferenciável;

- A família $\left\{\left(U_{\alpha}, \phi_{\alpha}\right)\right\}$ é máxima relativamente às condições acima e ela é chamada de estrutura diferenciável da variedade $M$.

Para simplificação do texto, usaremos $M^{n}$ para indicar que $M$ é uma variedade de dimensão $n$.

Definição 1.2.3. Sejam $M^{m}$ e $N^{n}$ variedades diferenciáveis. Uma aplicação diferenciável $\phi: M \rightarrow$ $N$ é uma imersão se $d \phi: T_{p} M \rightarrow T_{\phi(p)} N$ é injetiva para todo $p \in M$.

Note que, se $\phi: M^{m} \rightarrow N^{n}$ é uma imersão, então $m \leq n$, e a diferença entre $n$ e $m$ é chamada de codimensãa de $\phi$. 
Dois conceitos que estão relacionados ao de variedade diferenciável são os conceitos de fibrado vetorial e subfibrado, que serão definidos a seguir.

Definição 1.2.4 (Fibrado vetorial). Dada uma variedade diferenciável $M$, um fibrado vetorial (real suave) sobre $M$ é uma variedade diferenciável $E$, juntamente com uma aplicação diferenciável e sobrejetiva $\pi: E \rightarrow M$ satisfazendo as seguintes condições:

(a) Existe $k \in \mathbb{N}$ tal que, para todo $p \in M$, o conjunto $E_{p}=\pi^{-1}(p)$ possui uma estrutura de espaço vetorial real $k$-dimensional.

(b) Para cada $p \in M$, existem uma vizinhança $U \subset M$ de $p$ e uma aplicação diferenciável $\Phi$ : $\pi^{-1}(U) \rightarrow U \times \mathbb{R}^{k}$ tais que:

- Para cada $q \in U$, a restrição de $\Phi$ a $E_{q}$ é um isomorfismo linear entre $E_{q}$ e $\{q\} \times \mathbb{R}^{k}$, munido com a estrutura canônica de espaço vetorial real.

- Se $\pi_{U}: U \times \mathbb{R}^{k} \rightarrow U$ denota a projeção sobre o primeiro fator, então $\pi=\pi_{U} \circ \Phi: \pi^{-1}(U) \rightarrow U$

Definição 1.2.5 (Subfibrado vetorial). Sejam E e F fibrados vetoriais sobre uma variedade diferenciável $M$, com $E \subset F$. Se $\pi_{E}=\left(\pi_{F}\right)_{\mid E}$, então dizemos que $E$ é um subfibrado de $F$.

As definições acima foram retiradas de [21] Algumas noções básicas são bem conhecidas da geometria Riemanniana, e podem ser estendidas ao ambiente pseudo-riemanniano, com isso, a lista abaixo expõe tais conceitos de forma sucinta:

Definição 1.2.6 (Métrica Riemmaniana). Uma métrica em uma variedade diferenciável é uma correspondência que associa a cada ponto $p$ em $M$ uma forma bilinear simétrica no espaço tangente $T_{p} M$, que varia diferenciavelmente de acordo com o ponto escolhido.

Uma métrica é dita Riemanniana se a forma bilinear é também positiva definida.

O conceito de métrica pode ser estendido ao ambiente pseudo-Riemanniano, onde uma métrica é dita pseudo-Riemanniana se a forma bilinear não é necessariamente positiva definida.

Definição 1.2.7 (Índice de uma variedade). Seja d a métrica de $M$. Fixado um ponto $p \in M, o$ indice de uma variedade $M$ é a dimensão do maior subespaço vetorial $V \subset T_{p} M$ tal que a métrica $\left.d\right|_{V}$ é negativa definida.

De fato, em dimensão finita, o índice de uma variedade será o número de entradas negativas da métrica que a acompanha.

As definições a seguir vêm com o intuito de estender o conceito de derivação sobre variedades, onde um cuidado maior é necessário para que nenhuma inconsistência exista:

Definição 1.2.8 (Conexões). Uma conexão afim $\nabla$ em uma variedade diferenciável $M$ é uma aplicação que leva dois campos de vetores em outro campo de vetor, indicada por $(X, Y) \stackrel{\nabla}{\rightarrow} \nabla_{X} Y$ e que satisfaz as seguintes propriedades:

- $\nabla_{f X+g Y} Z=f \nabla_{X} Z+g \nabla_{Y} Z$,

- $\nabla_{X}(Y+Z)=\nabla_{X} Y+\nabla_{X} Z$, 
- $\nabla_{X}(f Y)=f \nabla_{X} Y+X(f) Y$,

onde X.Y e Z são campos de vetores diferenciáveis e f e $g$ são funções reais diferenciáveis.

Definição 1.2.9. Uma conexão $\nabla$ em uma variedade Riemanniana $M$ é compativel com a métrica se e só se

$$
X(Y, Z)=\left(\nabla_{X} Y, Z\right)+\left(Y, \nabla_{X} Z\right) .
$$

Em posse de tais definições, prova-se o teorema que nos dá a existência de uma conexão ïdeal p̈ara o desenvolvimento da teoria:

Teorema 1.2.10 (Levi-Civita). Dada uma variedade Riemanniana $M$, existe uma única conexão afim $\nabla$ em $M$ satisfazendo as condições:

- $\nabla$ é simétrica.

- $\nabla$ é compativel com a métrica Riemanniana.

A prova será omitida, pois não acrescenta nada ao trabalho e pode ser encontrada em qualquer livro básico sobre a teoria. Tal conexão é conhecida como conexão de Levi-Civita.

Com esse resultado em mãos, a partir de agora toda conexão será a considerada a conexão de Levi-Civita da variedade $M$.

\subsection{Curvaturas}

Nesta seção daremos algumas definições sobre o conceito de curvatura e seus tipos principais, onde cada um desenvolve papel importante no avanço da teoria e representa muito para a compreensão de certos resultados obtidos.

Definição 1.3.1 (Curvatura). A curvatura $R$ de uma variedade Riemanniana $M$ é uma correspondência que associa para cada ponto $p \in M$ e cada par de campos de vetores $X, Y \in T_{p} M$, uma aplicação $R$ que recebe outro vetor, definida como:

$$
R(X, Y) Z=\nabla_{Y} \nabla_{X} Z-\nabla_{X} \nabla_{Y} Z+\nabla_{[X, Y]} Z
$$

onde $Z$ é um campo de vetor diferenciável em $M$ e $\nabla$ é a conexão de Levi-Civita de $M$.

Agora iremos definir a noção de curvatura seccional, e para tal enunciaremos uma proposição curta:

Proposição 1.3.2. Seja $\sigma \subset T_{p} M$ um subespaço bi-dimensional do espaço tangente $T_{p} M$ e sejam $x, y \in \sigma$ dois vetores linearmente independentes. Então

$$
K(x, y)=\frac{(x, y, x, y)}{|x \wedge y|^{2}},
$$

onde $(x, y, x, y)=<R(x, y) x, y>$, não depende da escolha dos vetores $x, y \in \sigma$.

Sua demonstração também pode encontrada em qualquer livro sobre a teoria. Com isso, podemos definir o conceito de curvatura seccional. 
Definição 1.3.3 (Curvatura seccional). Dado um ponto $p \in M$ e $\sigma \subset T_{p} M$ um subespaço bidimensional, o numero real $K(x, y)=K(\sigma)$, onde $x, y$ é uma base qualquer de $\sigma$, é denominado curvatura seccional de $\sigma$ em $p$.

Aqui definiremos os conceitos de curvatura de Ricci e escalar da maneira clássica, mas faremos o uso de sua expressão em coordenadas, que será apresentado mais a fente.

Definição 1.3.4 (Curvatura de Ricci). Fixado $x=z_{n}$ um vetor unitário em $T_{p} M$. Tomemos uma base ortonormal $z_{1}, \ldots, z_{n-1}$ de um subespaço de $T_{p} M$ ortogonal a $x$, então a curvatura de Ricci na direção $x$ é dada por

$$
\operatorname{Ric}_{p}(x)=\frac{1}{n-1} \sum_{i}<R\left(x, z_{i}\right) x, z_{i}>, i=1, \ldots, n-1 .
$$

Definição 1.3.5 (Curvatura média ou escalar). Como seu próprio nome já diz, a curvatura média é uma média da curvatura de Ricci e é definida como

$$
K(p)=\frac{1}{n} \sum_{j} \operatorname{Ric}_{p}\left(z_{j}\right)
$$

Aqui daremos um exemplo de tensor em uma variedade Riemanniana, que chamaremos de tensor curvatura, onde no caso, tensor é simplesmente uma aplicação multilinear, que será definida com mais detalhes ao longo do trabalho.

Definição 1.3.6 (Tensor Curvatura). O tensor curvatura recebe como entrada 4 campos de vetores diferenciáveis, nos dando uma função diferenciável como saída, e é definido por

$$
R\left(e_{i}, e_{j}, e_{k}, e_{l}\right)=<R\left(e_{i}, e_{j}\right) e_{k}, e_{l}>=R_{i j k l}
$$

Onde $\left\{e_{i}\right\}$ é um sistema de coordenadas.

\subsection{Os espaços $L_{p}^{n+p}$ e a segunda forma fundamental $I I_{\eta}$.}

O espaço $L_{p}^{n+p}$ é chamado de espaço de Lorentz, ou seja, uma variedade pseudo-Riemanniana de índice p. Quando o espaço $L_{p}^{n+p}$ é simplesmente conexo e possui curvatura seccional constante $c$, então ele é chamado de espaço forma de Lorentz e é denotado por $L_{p}^{n+p}(c)$. Uma classe importante de espaços-forma muito estudada são os espaços de Lorentz-Minkowski ( $c=0, p=1)$, o espaço de Sitter $(c>0, p=1)$ e o espaço anti-de Sitter $(c<0, p=1)$, e são denotados respectivamente por $\mathbb{L}_{1}^{n+1}, \mathbb{S}_{1}^{n+1}$ e $\mathbb{H}_{1}^{n+1}$.

O espaço objeto de estudo neste trabalho é o $\mathbb{H}_{1}^{n+1}$ e suas hipersuperfícies tipo espaço imersas. Vale relembrar que uma hipersuperfície é tipo espaço quando a métrica induzida pela imersão no espaço $L_{p}^{n+p}$ for positiva definida.

Seja $c$ uma constante positiva, de maneira geral, o espaço $L_{p}^{n+p}$ pode ser definido da seguinte maneira

$$
L_{p}^{n+p}(c)=\left\{\left(x_{1}, x_{2}, \ldots, x_{n+p+1}\right) \in \mathbb{R}_{p+1}^{n+p+1}: \sum_{i=1}^{n} x_{i}^{2}-\sum_{j=n+1}^{n+p+1} x_{j}^{2}=c\right\},
$$


onde $\mathbb{R}_{p+1}^{n+p+1}$ é o espaço vetorial real de dimensão $(\mathrm{n}+\mathrm{p}+1)$ munido de um produto interno com índice $p+1$ dado por $\langle x, y\rangle=\sum_{i=1}^{n} x_{i} y_{i}-\sum_{j=n+1}^{n+p+1} x_{j} y_{j}$.

Dois dos espaços-forma centrais na teoria de subvariedades são o espaço anti-de Sitter e o espaço de Sitter, e são definidos a seguir.

O espaço anti-de Sitter $\mathbb{H}_{1}^{n+1}$ é definido como

$$
\mathbb{H}_{1}^{n+1}=\left\{\left(x_{1}, x_{2}, \ldots, x_{n+2}\right) \in \mathbb{R}_{2}^{n+2}: \sum_{i=1}^{n} x_{i}^{2}-\sum_{j=n+1}^{n+2} x_{j}^{2}=c\right\},
$$

Já o espaço de Sitter

$$
\mathbb{S}_{1}^{n+1}=\left\{\left(x_{1}, x_{2}, \ldots, x_{n+2}\right) \in \mathbb{R}_{1}^{n+2}: \sum_{i=1}^{n+1} x_{i}^{2}-x_{n+2}^{2}=c\right\},
$$

e também é alvo de estudos na área de classificações hipersuperfícies.

\subsubsection{A segunda forma fundamental e suas consequências}

Um conceito muito importante no estudo de imersões é o da segunda forma fundamental. Vamos definir a segunda forma fundamental da imersão $f: M \rightarrow \bar{M}$, onde $M$ e $\bar{M}$ são variedades diferenciáveis. Para isto, convém introduzir previamente a seguinte definição. Se $X$ e $Y$ são campos de vetores locais em $M$, para cada $p \in M$ a aplicação $B: T_{p} M \times T_{p} M \rightarrow T_{p} \bar{M}$ definida por

$$
B(X, Y)=\bar{\nabla}_{\bar{X}} \bar{Y}-\nabla_{X} Y
$$

onde $\nabla$ e $\bar{\nabla}$ são as conexões de $M$ e $\bar{M}$ respectivamente, e $\bar{X}, \bar{Y}$ são extensões dos campos de vetores $X$ e $Y$ tangentes a $M$ tais que $X(p)=x$ e $Y(p)=y$, é um campo local em $\bar{M}$ normal a $M . B(X, Y)$ não depende das extensões $\bar{X}, \bar{Y}$, e tal fato pode ser verificado facilmente por cálculo direto, pois, se $\bar{X}_{1}$ é uma outra extensão de $X$, teremos

$$
\left(\bar{\nabla}_{\bar{X}} \bar{Y}-\nabla_{X} Y\right)-\left(\bar{\nabla}_{\bar{X}_{1}} \bar{Y}-\nabla_{X} Y\right)=\bar{\nabla}_{\bar{X}-\overline{X_{1}}} \bar{Y}
$$

que se anula em $M$, pois $\bar{X}-\overline{X_{1}}=0$ em $M$; alem disto, se $\bar{Y}_{1}$ é uma outra extensão de $Y$,

$$
\left(\bar{\nabla}_{\bar{X}} \bar{Y}-\nabla_{X} Y\right)-\left(\bar{\nabla}_{\bar{X}} \bar{Y}_{1}-\nabla_{X} Y\right)=\bar{\nabla}_{\bar{X}}\left(\bar{Y}-\bar{Y}_{1}\right)=0
$$

pois $\bar{Y}-\bar{Y}_{1}=0$ ao longo de uma trajetória de $X$.Portanto $B(X, Y)$ está bem definida.

Agora, veremos que $B(X, Y)$ é uma aplicação bilinear e simétrica, enunciado como uma proposição, o que é muito importante, pois tal fato torna a segunda forma fundamental, que será definida posteriormente, a ser também uma forma bilinear e simétrica.

Proposição 1.4.1. Se $X, Y$ são campos de vetores locais em $T_{p} M$, então a aplicação

$$
B(X, Y)=\bar{\nabla}_{\bar{X}} \bar{Y}-\nabla_{X} Y
$$

é bilinear e simetrica. 
Demonstração. A aditividade de $B$ é obtida facilmente através da linearidade da conexão Riemanniana e também conclui-se que, fixada uma função $f$ diferenciável localmente em $M$, temos que $B(f X, Y)=f B(X, Y)$. Portanto falta mostrar que $B(X, f Y)=f B(X, Y)$. Indicando por $\bar{f}$ uma extensão de $f$ teremos

$$
\begin{aligned}
B(X, f Y) & =\bar{\nabla}_{\bar{X}}(\bar{f} Y)-\nabla_{X}(f Y) \\
& =\bar{f}_{\bar{X}} \bar{Y}-f \nabla_{X} Y+\bar{X}(\bar{f}) \bar{Y}-X(f) Y .
\end{aligned}
$$

Como em $M, f=\bar{f}$ e $\bar{X}(\bar{f})=X(f)$, concluímos que as duas últimas parcelas se anulam, e com isso temos $B(X, f Y)=f B(X, Y)$, logo, $B$ é bilinear.

Para mostrar que $B$ é simétrica, faremos uso da simetria da conexão Riemanniana, de modo que

$$
B(X, Y)=\bar{\nabla}_{\bar{X}} \bar{Y}-\nabla_{X} Y=\bar{\nabla}_{\bar{Y}} \bar{X}+[\bar{X}, \bar{Y}]-\nabla_{Y} X-[X, Y] .
$$

Como em $M,[\bar{X}, \bar{Y}]=[X, Y]$, concluímos que $B(X, Y)=B(Y, X)$.

Agora, podemos ver que, para cada $p \in M$, e $\eta \in\left(T_{p} M\right)^{\perp}$, a aplicação $H_{\eta}: T_{p} M \times T_{p} M \rightarrow \mathbb{R}$ definida por

$$
H_{\eta}(x, y)=<B(x, y), \eta>, \quad x, y \in T_{p} M,
$$

é, de acordo com a Proposição 1.4.1, uma forma bilinear e simétrica.

Definição 1.4.2 (Segunda Forma Fundamental). A forma quadrática $I I_{\eta}$ definida em $T_{p} M$ por

$$
I I_{\eta}(x)=H_{\eta}(x, x)
$$

é chamada de segunda forma fundamental de $f$ em $p$ segundo o vetor normal $\eta$.

Vale relembrar que, para cada $p \in M$, o espaço tangente $T_{p} \bar{M}$ pode ser decomposto da seguinte forma

$$
T_{p} \bar{M}=T_{p} M \oplus\left(T_{p} M\right)^{\perp},
$$

onde $\left(T_{p} M\right)^{\perp}$ é o complemento ortogonal de $T_{p} M$ em $T_{p} \bar{M}$.

É importante observar que, a aplicação $H_{\eta}$ pode ser associada a uma aplicação linear autoadjunta $S_{\eta}: T_{p} M \rightarrow T_{p} M$ por

$$
<S_{\eta}(x), y>=H_{\eta}(x, y)=<B(x, y), \eta>.
$$

Em forma de uma proposição, daremos uma expressão da aplicação $S_{\eta}$ em termos da derivada covariante.

Proposição 1.4.3. Seja $p \in M, x \in T_{p} M$ e $\eta \in\left(T_{p} M\right)^{\perp}$. Seja $N$ uma extensão local de $\eta$ normal a M. Então

$$
S_{\eta}(x)=-\left(\bar{\nabla}_{x} N\right)^{T}
$$

onde $\left(\bar{\nabla}_{x} N\right)^{T}$ denota a componente tangencial de $\bar{\nabla}_{x} N$. 
Demonstração. Seja $y \in T_{p} M$ e $X, Y$ extensões locais de $x, y$, respectivamente, e tangentes a $M$. Então $\langle N, Y\rangle=0$, e portanto

$$
\begin{aligned}
<S_{\eta}(x), y>= & <B(X, Y)(p), N>=<\bar{\nabla}_{X} Y-\nabla_{X} Y, N>(p) \\
= & <\bar{\nabla}_{X} Y, N>(p)=-<Y, \bar{\nabla}_{X} N>(p)=<-\bar{\nabla}_{x} N, y>
\end{aligned}
$$

e como é válido para todo $y \in T_{p} M$, segue a tese.

Uma observação que vale ser feita para a aplicação $S_{\eta}$. Considerando o caso de hipersuperfícies, ou seja, onde a codimensão de $M$ é 1 , fixe $p \in M$ e $\eta \in T_{p} M$ tal que $|\eta|=1$. dado que $S_{\eta}$ : $T_{p} M \rightarrow T_{p} M$ é simétrica (pela sua definição e propriedade da aplicação $B$ ), então existe uma base ortonormal de vetores próprios $\left\{e_{1}, \ldots, e_{n}\right\}$ de $T_{p} M$ com valores próprios reais $\lambda_{1}, \ldots, \lambda_{n}$, isto é, $S_{\eta}\left(e_{i}\right)=\lambda_{i} e_{i}$. Tal base é importante e será utilizada extensamente na obtensão dos resultados apresentados neste trabalho.

Agora apresentaremos um lema, omitindo sua demonstração, mas com o objetivo de obter uma expressão melhor para a curvatura seccional de uma variedade pseudo-Riemmaniana. O lema será enunciado para $M$ sendo uma variedade Riemanniana, mas o resultado para o caso pseudoRiemanniano também é valido.

Lema 1.4.4. Sejam $M$ uma variedade Riemanniana e $p$ um ponto de $M$. Defina uma aplicação trilinear $R^{\prime}: T_{p} M \times T_{p} M \times T_{p} M \rightarrow T_{p} M$ por

$$
<R^{\prime}(X, Y) W, Z>=<X, W><Y, Z>-<Y, W><X, Z>
$$

para todo $X, Y, W, Z \in T_{p} M$. Então $M$ tem curvatura seccional constante igual a $K_{0}$ se e só se $R=K_{0} R^{\prime}$, onde $R$ é a curvatura de $M$.

Demonstração. Ver [14]

Com tal resultado em mãos, e sabendo que no caso do espaço $\mathbb{H}_{1}^{n+1}(-1)$, temos $K_{0}=-1$, podemos fixar um ponto $p \in \mathbb{H}_{1}^{n+1}$ e tomar uma base ortonormal $\left\{e_{i}\right\}_{n+1}$ local em $p$, tal que $<e_{A}, e_{B}>=\epsilon_{A} \delta_{A B}$, então temos

$$
\begin{aligned}
R_{A B C D} & =K_{0} R_{A B C D}^{\prime} \\
& =(-1)<e_{A}, e_{C}><e_{B}, e_{D}>-<e_{B}, e_{C}><e_{A}, e_{D}> \\
& =(-1)\left(\epsilon_{A} \delta_{A C} \epsilon_{B} \delta_{B D}-\epsilon_{B} \delta_{B C} \epsilon_{A} \delta_{A D}\right) \\
& =-\epsilon_{A} \epsilon_{B}\left(\delta_{A C} \delta_{B D}-\delta_{B C} \delta_{A D}\right) .
\end{aligned}
$$

\subsection{Distribuições e formas diferencias}

O objetivo desta seção é apresentar algumas definições e resultados básicos sobre distribuições, envolvendo formas diferenciais, que são amplamente usadas neste trabalho, e é ferramenta indispensável para o entendimento de um dos teoremas chave deste trabalho. 
Definição 1.5.1 (Distribuição). Seja $M$ uma variedade com estrutura topológica e diferenciável. Uma escolha de um subespaço linear, $k$-dimensional $D_{p} \subset T_{p} M$, para cada ponto $p \in M$, é chamado de uma distribuição tangente $k$-dimensional em $M$, ou simplesmente, uma distribuição.

Definição 1.5.2 (Distribuição suave). Uma distribuição é dita suave se a união de todos os subespaços $D_{p}$ formam um subfibrado suave $D=\bigcup_{p \in M} D_{p} \subset T M$.

Definição 1.5.3 (Variedade integral). Suponha que $D \subset T M$ seja uma distribuiçấo suave. Uma subvariedade imersa $N \subset M$ é chamada de variedade integral de $D$ se $T_{p} N=D_{p}$, para cada ponto $p \in M$.

Definição 1.5.4 (Distribuição completamente integrável). Uma distribuição D é dita integrável se, cada ponto de $M$ está contido em uma variedade integral de D.

Proposição 1.5.5. Seja $D$ uma distribuição numa variedade $C^{\infty} M$. Se $D$ é completamente integrável, entao para todos vetores $X, Y \in D$, o colchete $[X, Y]$ também pertence a $D$.

A demonstração da proposição acima enontra-se em [20]. Tal proposição motiva a próxima definição:

Definição 1.5.6 (Involutividade). Uma distribuição $D$ em uma variedade $M, C^{\infty}$, é dita involutiva se o colchete $[X, Y]$, onde $X$ e $Y$ são dois vetores arbitrários pertencentes a $D$, também pertencer a D.

Um dos resultados mais conhecidos da teoria de distribuições, é conhecido como Teorema de Frobenius, e tem diversas aplicações em muitos campos da matemática.

Teorema 1.5.7 (Frobenius). Uma condição necessária e suficiente para uma distribuição D numa variedade $C^{\infty}, M$ seja completamente integrável, é que $D$ seja involutiva.

Para finalizar, enunciaremos a definição de distribuição paralela, que será utilizada posteriormente:

Definição 1.5.8 (Distribuiçao paralela). Seja $M$ uma variedade pseudo-riemanniana. Supondo que, dada uma divisão do fibrado tangente na forma $T M=V_{1} \oplus V_{2}$, onde $V_{1}$ e $V_{2}$ são subfibrados suaves tangentes que são chamados de distribuiçôes. Isto define duas projeções complementares $\pi_{1} e$ $\pi_{2}$ de $T M$ em $V_{1}$ e $V_{2}$. Dizemos que $V_{1}$ é uma distribuição paralela se $\nabla \pi_{1}=0$. Equivalentemente, isto significa que, se para todo $X_{1}$, campo de vetores $C^{\infty}$ que assume valores em $V_{1}$, então $\nabla X_{1}$ também assume valores em $V_{1}$. 


\section{Capítulo 2}

\section{Preliminares}

Neste capítulo apresentaremos alguns conceitos básicos que serão utilizados ao longo deste trabalho.

\subsection{O referencial móvel em $\mathbb{H}_{1}^{n+1}$}

Seja $M^{n}$ uma hipersuperfície tipo espaço no espaço anti-de Sitter $\mathbb{H}_{1}^{n+1}$. Fixe um aberto $U \subset M^{n}$, para cada $p \in U$, fixamos um referencial ortonormal local pseudo-Riemanniano $\left\{e_{1}, \ldots, e_{n+1}\right\}$ em $\mathbb{H}_{1}^{n+1}$, tal que $e_{1}, \ldots, e_{n}$ são tangentes a $M^{n}$. No que segue usaremos a seguinte convenção para os índices:

$$
\begin{gathered}
1 \leq A, B, C, \cdots \leq n+1, \\
1 \leq i, j, k, \cdots \leq n,
\end{gathered}
$$

Sejam também as 1-formas $\omega_{i}$ que formam o referencial dual ao referencial $\left\{e_{i}\right\}$, ou seja, $\omega_{i}\left(e_{j}\right)=\delta_{i j}$ onde $\delta_{i j}$ é o delta de Kronecker. A métrica pseudo-Riemanniana de $\mathbb{H}_{1}^{n+1}$ é dada por

$$
d s^{2}=\sum_{A} \epsilon_{A} \omega_{A}^{2}
$$

onde fixamos $\epsilon_{n+1}=-1$ e $\epsilon_{i}=1, \forall i \leq n$.

Usando a definição de conexão, podemos escreve-la na base $\left\{e_{i}\right\}$ da seguinte maneira:

$$
\nabla_{e_{A}} e_{B}=\sum_{C}^{n+1} \epsilon_{C} \omega_{B C}\left(e_{A}\right) e_{C} .
$$

As 1-formas $\omega_{i j}$ serão denominadas de formas de conexão.

Definição 2.1.1 (Formas de curvatura). Dados $v, w$ vetores tangentes, seja $\left[\Omega_{j i}\right]$ a matriz do operador curvatura $R_{v w}$ relativa a base $\left\{e_{i}\right\}$, tais operadores são definidos através das relações:

- $R_{v w}\left(e_{j}\right)=\sum_{i} \epsilon_{i} \Omega_{j i}(v, w) e_{i}$

- $<R_{i j}\left(e_{k}\right), e_{l}>=\epsilon_{l} R_{i j k l}$

Com isso, temos que:

$$
\epsilon_{i} R_{i j k l}=\epsilon_{i} \Omega_{j i}\left(e_{k}, e_{l}\right) .
$$


Usando que uma 2-forma pode ser escrita como combinação de elementos da base, concluímos que:

$$
\Omega_{j i}=\frac{1}{2} \sum_{r, s} \epsilon_{r} \epsilon_{s} R_{i j r s} \omega_{r} \wedge \omega_{s}
$$

Teorema 2.1.2 (Primeira equação de estrutura).

$$
\begin{gathered}
\omega_{A B}=-\epsilon_{A} \epsilon_{B} \omega_{B A}, \\
d \omega_{A}=\sum_{B} \epsilon_{B} \omega_{A B} \wedge \omega_{B} .
\end{gathered}
$$

Demonstração. Primeiramente, basta observar que $\left\langle e_{A}, e_{B}\right\rangle=\epsilon_{A} \delta_{A B}$, assim, vamos derivar em uma direção $v$ qualquer, e usando a regra de Leibniz temos:

$$
\begin{aligned}
\nabla_{(v)}\left(<e_{A}, e_{B}>\right) & =<\nabla_{(v)} e_{A}, e_{B}>+<e_{A}, \nabla_{(v)} e_{B}> \\
& =\epsilon_{B} \omega_{A B}+\epsilon_{A} \omega_{B A} \\
& =0
\end{aligned}
$$

Para provar a segunda igualdade, vamos calcular ambos os lados em dois vetores da base quaisquer $e_{i}, e_{j}$ e notar que eles chegam na mesma expressão.

$$
\begin{aligned}
d \omega_{A}\left(e_{i}, e_{j}\right) & =e_{i}\left(\omega_{A}\left(e_{j}\right)\right)-e_{j}\left(\omega_{A}\left(e_{i}\right)\right)-\omega_{A}\left(\left[e_{i}, e_{j}\right]\right) \\
& =-\omega_{A}\left(\left[e_{i}, e_{j}\right]\right) \\
& =-\omega_{A}\left(\nabla_{e_{i}} e_{j}-\nabla_{e_{j}} e_{i}\right) \\
& =\omega_{A}\left(\nabla_{e_{j}} e_{i}\right)-\omega_{A}\left(\nabla_{e_{i}} e_{j}\right) \\
& =\omega_{A}\left[\sum_{k} \epsilon_{k} \omega_{i k}\left(e_{j}\right) e_{k}\right]-\omega_{A}\left[\sum_{k} \epsilon_{k} \omega_{j k}\left(e_{i}\right) e_{k}\right] \\
& =\sum_{k} \epsilon_{k} \omega_{i k}\left(e_{j}\right) \omega_{A}\left(e_{k}\right)-\sum_{k} \epsilon_{k} \omega_{j k}\left(e_{i}\right) \omega_{A}\left(e_{k}\right) \\
& =\epsilon_{A} \omega_{i A}\left(e_{j}\right) \epsilon_{A}-\epsilon_{A} \omega_{j A}\left(e_{i}\right) \epsilon_{A} \\
& =\omega_{i A}\left(e_{j}\right)-\omega_{j A}\left(e_{i}\right) \\
& =\omega_{A j}\left(e_{i}\right)-\omega_{A i}\left(e_{j}\right) .
\end{aligned}
$$

Desenvolvendo o lado direito, calculado nos mesmos $e_{i}, e_{j}$ : 


$$
\begin{aligned}
\sum_{B} \epsilon_{B} \omega_{A B} \wedge \omega_{B}\left(e_{i}, e_{j}\right) & =\sum_{B} \epsilon_{B} \omega_{A B}\left(e_{i}\right) \omega_{B}\left(e_{j}\right)-\sum_{B} \epsilon_{B} \omega_{A B}\left(e_{j}\right) \omega_{B}\left(e_{i}\right) \\
& =\sum_{B} \epsilon_{B} \omega_{A B}\left(e_{i}\right) \epsilon_{B} \delta_{B j}-\sum_{B} \epsilon_{B} \omega_{A B}\left(e_{j}\right) \epsilon_{B} \delta_{B i} \\
& =\epsilon_{j}^{2} \omega_{A j}\left(e_{i}\right)-\epsilon_{i}^{2} \omega_{A i}\left(e_{j}\right) \\
& =\omega_{A j}\left(e_{i}\right)-\omega_{A i}\left(e_{j}\right) . \\
& \therefore d \omega_{A}=\sum_{B} \epsilon_{B} \omega_{A B} \wedge \omega_{B} .
\end{aligned}
$$

Teorema 2.1.3 (Segunda equação de estrutura).

$$
d \omega_{A B}=\sum_{C} \epsilon_{C} \omega_{A C} \wedge \omega_{C B}-\frac{1}{2} \sum_{C, D} \epsilon_{C} \epsilon_{D} R_{A B C D} \omega_{C} \wedge \omega_{D}
$$

Demonstração. De acordo com as definições, faremos o cálculo da curvatura nos vetores da base $\left\{e_{i}\right\}$ :

$$
R_{i j}\left(e_{A}\right)=\nabla_{e_{j}} \nabla_{e_{i}} e_{A}-\nabla_{e_{i}} \nabla_{e_{j}} e_{A}+\nabla_{\left[e_{i} \cdot e_{j}\right]} e_{A} .
$$

Analisando separadamente cada termo temos que:

$$
\begin{aligned}
\nabla_{e_{j}} \nabla_{e_{i}} e_{A} & =\nabla_{e_{j}}\left(\sum_{B} \epsilon_{B} \omega_{A B}\left(e_{i}\right) e_{B}\right) \\
& =\sum_{B} e_{j}\left[\epsilon_{B} \omega_{A B}\left(e_{i}\right)\right] e_{B}+\sum_{B} \epsilon_{B} \omega_{A B}\left(e_{i}\right) \nabla_{e_{j}} e_{B} \\
& =\sum_{B} e_{j}\left[\epsilon_{B} \omega_{A B}\left(e_{i}\right)\right] e_{B}+\sum_{B, C} \epsilon_{B} \omega_{A B}\left(e_{i}\right) \epsilon_{C} \omega_{B C}\left(e_{j}\right) e_{C} \\
& =\sum_{B} e_{j}\left[\epsilon_{B} \omega_{A B}\left(e_{i}\right)\right] e_{B}+\sum_{B, C} \epsilon_{B} \epsilon_{C} \omega_{A B}\left(e_{i}\right) \omega_{B C}\left(e_{j}\right) e_{C} .
\end{aligned}
$$

Analogamente, para o segundo membro, haverá somente a permutação dos indices $i$ e $j$ : 


$$
\begin{aligned}
\nabla_{e_{i}} \nabla_{e_{j}} e_{A} & =\nabla_{e_{i}}\left(\sum_{B} \epsilon_{B} \omega_{A B}\left(e_{j}\right) e_{B}\right) \\
& =\sum_{B} e_{i}\left[\epsilon_{B} \omega_{A B}\left(e_{j}\right)\right] e_{B}+\sum_{B} \epsilon_{B} \omega_{A B}\left(e_{j}\right) \nabla_{e_{i}} e_{B} \\
& =\sum_{B} e_{i}\left[\epsilon_{B} \omega_{A B}\left(e_{j}\right)\right] e_{B}+\sum_{B, C} \epsilon_{B} \omega_{A B}\left(e_{j}\right) \epsilon_{C} \omega_{B C}\left(e_{i}\right) e_{C} \\
& =\sum_{B} e_{i}\left[\epsilon_{B} \omega_{A B}\left(e_{j}\right)\right] e_{B}+\sum_{B, C} \epsilon_{B} \epsilon_{C} \omega_{A B}\left(e_{j}\right) \omega_{B C}\left(e_{i}\right) e_{C} .
\end{aligned}
$$

Para o terceiro membro, temos:

$$
\nabla_{\left[e_{i}, e_{j}\right]} e_{A}=\sum_{B} \epsilon_{B} \omega_{A B}\left(\left[e_{i}, e_{j}\right]\right) e_{B}
$$

Substituindo em (2.4) as expressões encontradas e agrupando as somas temos que:

$$
\begin{aligned}
R_{i j}\left(e_{A}\right)= & -\sum_{B}\left[\epsilon_{B} e_{i} \omega_{A B}\left(e_{j}\right)-\epsilon_{B} e_{j} \omega_{A B}\left(e_{i}\right)-\epsilon_{B} \omega_{A B}\left(\left[e_{i}, e_{j}\right]\right)\right] e_{B} \\
& +\sum_{B, C}\left[\epsilon_{B} \epsilon_{C} \omega_{A B}\left(e_{i}\right) \omega_{B C}\left(e_{j}\right)-\epsilon_{B} \epsilon_{C} \omega_{A B}\left(e_{j}\right) \omega_{B C}\left(e_{i}\right)\right] e_{C} .
\end{aligned}
$$

Rearranjando os índices $B$ e $C$ no segundo somatório, segue que:

$$
\begin{aligned}
R_{i j}\left(e_{A}\right)= & -\sum_{B} \epsilon_{B}\left[e_{i} \omega_{A B}\left(e_{j}\right)-e_{j} \omega_{A B}\left(e_{i}\right)-\omega_{A B}\left(\left[e_{i}, e_{j}\right]\right)\right] e_{B} \\
& +\sum_{B, C} \epsilon_{B} \epsilon_{C}\left[\omega_{A C}\left(e_{i}\right) \omega_{C B}\left(e_{j}\right)-\omega_{A C}\left(e_{j}\right) \omega_{C B}\left(e_{i}\right)\right] e_{B} .
\end{aligned}
$$

Portanto:

$$
\begin{aligned}
R_{i j}\left(e_{A}\right) & =-\sum_{B} \epsilon_{B} d \omega_{A B}\left(e_{i}, e_{j}\right) e_{B}+\sum_{B, C} \epsilon_{B} \epsilon_{C} \omega_{A C} \wedge \omega_{C B}\left(e_{i}, e_{j}\right) e_{B} \\
& =-\sum_{B} \epsilon_{B}\left[d \omega_{A B}-\sum_{C} \epsilon_{C} \omega_{A C} \wedge_{C B}\right]\left(e_{i}, e_{j}\right) e_{B} .
\end{aligned}
$$

Logo pela definição 2.1.1 segue que

$$
\begin{aligned}
\sum_{B} \epsilon_{B} \Omega_{A B}\left(e_{i}, e_{j}\right) e_{B} & =-\sum_{B} \epsilon_{B}\left[d \omega_{A B}-\sum_{C} \epsilon_{C} \omega_{A C} \wedge_{C B}\right]\left(e_{i}, e_{j}\right) e_{B}, \\
\Omega_{A B} & =-d \omega_{A B}+\sum_{C} \epsilon_{C} \omega_{A C} \wedge \omega_{C B},
\end{aligned}
$$




$$
\begin{gathered}
d \omega_{A B}=-\Omega_{A B}+\sum_{C} \epsilon_{C} \omega_{A C} \wedge \omega_{C B} \\
d \omega_{A B}=\sum_{C} \epsilon_{C} \omega_{A C} \wedge \omega_{C B}-\frac{1}{2} \sum_{C, D} \epsilon_{C} \epsilon_{D} R_{A B C D} \omega_{C} \wedge \omega_{D} .
\end{gathered}
$$

A partir deste ponto, vale frisar que, como $M^{n}$ é uma hipersuperfície tipo espaço, então temos que as equações de estrutura ficam:

$$
\begin{gathered}
d \omega_{i}=\sum_{j} \omega_{i j} \wedge \omega_{j}, \quad \omega_{i j}=\omega_{j i}, \\
d \omega_{i j}=\sum_{k} \omega_{i k} \wedge \omega_{k j}-\frac{1}{2} \sum_{k l} R_{i j k l} \omega_{k} \wedge \omega_{l} .
\end{gathered}
$$

Agora vamos enunciar um dos resultados mais importantes na teoria do referencial móvel, que garante a consistência do método.

Lema 2.1.4 (Lema de Cartan). Seja $V$ um espaço vetorial de dimensão $n$, e sejam $\omega_{1}, \ldots, \omega_{r}$ : $V \rightarrow \mathbb{R}, r \leq n$, formas lineares em $V$, que são linearmente independentes. Admita que existam formas lineares $\theta_{1}, \ldots, \theta_{r}: V \rightarrow \mathbb{R}$ tais que $\sum_{i=1}^{r} \omega_{i} \wedge \theta i=0$. Então

$$
\theta_{i}=\sum_{j} a_{i j} \omega_{j}, \quad a_{i j}=a_{j i}
$$

Demonstração. A partir das formas lineares $\omega_{i}$, complete-as para uma base $\omega_{1}, \ldots, \omega_{r}, \omega_{r+1}, \ldots, \omega_{n}$ de $V^{*}$, e escreva

$$
\theta_{i}=\sum_{j} a_{i j} \omega_{j}+\sum_{l} b_{i l} \omega_{l}, \quad l=r+1, \ldots, n .
$$

Usando que $\sum_{i=1}^{r} \omega_{i} \wedge \theta_{i}=0$ obtemos

$$
\begin{aligned}
0 & =\sum_{i=1}^{r} \omega_{i} \wedge \theta_{i}=\sum_{i j} a_{i j} \omega_{i} \wedge \omega_{j}+\sum_{i l} b_{i l} \omega_{i} \wedge \omega_{l} \\
& =\sum_{i<j}\left(a_{i j}-a_{j i}\right) \omega_{i} \wedge \omega_{j}+\sum_{i<l} b_{i l} \omega_{i} \wedge \omega_{l} .
\end{aligned}
$$

Como $\omega_{k} \wedge \omega_{s}$, com $k<s$ e $k, s=1, \ldots, n$, são linearmente independentes, concluímos que $b_{i l}=0$ e $a_{i j}=a_{j i}$.

\subsection{Segunda forma fundamental e a Equação De Gauss no método do referencial móvel}

O objetivo desta seção é introduzir conceitos ja conhecidos e definidos anteriormente, mas na linguagem do referencial móvel, que será usada durante a demonstração dos teoremas principais deste trabalho. 
Definição 2.2.1 (Segunda forma fundamental). A segunda forma fundamental, em termos do referencial móvel será definida como

$$
h=\sum_{i j} h_{i j} \omega_{i} \omega_{j}
$$

onde $h_{i j}$ são as componentes da matriz de $h$ na base $\left\{e_{i}\right\}$.

Definição 2.2.2 (Norma da Segunda Forma Fundamental). A norma de segunda forma fundamental será denotada por

$$
S=\sum_{i j} h_{i j}^{2}
$$

Definição 2.2.3 (Curvatura Média). A curvatura média de uma hipersuperfície $M^{n}$ é definida como

$$
H=\frac{1}{n} \sum_{i} h_{i i}
$$

De acordo com [14], a equação de Gauss tem a forma:

$$
<R^{M}(X, Y) Z, W>=<R^{N}(X, Y) Z, W>+h(Y, Z) h(X, W)-h(X, Z) h(Y, W)
$$

Lembrando que tal equação é válida ao redor de um ponto $p \in M$ e $X, Y, Z, W \in T_{p} M$ são campos de vetores diferenciáveis, $<R^{M}(X, Y) Z, W>$ é a curvatura da subvariedade e $<R^{N}(X, Y) Z, W>$ é a curvatura do espaço ambiente.

Em termos do referencial ortonormal móvel $\left\{e_{i}\right\}$, a equação de Gauss fica da seguinte maneira:

$$
<R^{M}\left(e_{i}, e_{j}\right) e_{k}, e_{l}>=<R^{N}\left(e_{i}, e_{j}\right) e_{k}, e_{l}>+h\left(e_{j}, e_{k}\right) h\left(e_{i}, e_{l}\right)-h\left(e_{i}, e_{k}\right) h\left(e_{j}, e_{l}\right),
$$

ou ainda

$$
R_{i j k l}=K_{i j k l}+h\left(e_{j}, e_{k}\right) h\left(e_{i}, e_{l}\right)-h\left(e_{i}, e_{k}\right) h\left(e_{j}, e_{l}\right) .
$$

Como $h\left(e_{i}, e_{j}\right)=\sum_{r, s} h_{r s} \omega_{r}\left(e_{i}\right) \omega_{s}\left(e_{j}\right)=\sum_{r, s} h_{r s} \delta_{r i} \delta_{s j}=h_{i j}$, temos então que:

$$
R_{i j k l}=-\epsilon_{i} \epsilon_{j}\left(\delta_{i k} \delta_{j l}-\delta_{i l} \delta_{j k}\right)+h_{j k} h_{i l}-k_{i k} h_{j l} .
$$

Mas como $i, j \leq n \Rightarrow \epsilon_{i} \epsilon_{j}=1$ temos:

$$
R_{i j k l}=\delta_{i l} \delta_{j k}-\delta_{i k} \delta_{j l}+h_{j k} h_{i l}-h_{i k} h_{j l} .
$$

\subsection{Componentes de $\nabla h$ e a equação de Codazzi}

Definição 2.3.1. Seja $F$ um tensor de ordem $r, F: \underbrace{T_{p} M \times \cdots \times T_{p} M}_{r \text { vezes }} \rightarrow \mathbb{R}$

$A$ diferencial covariante $\nabla F$ é um tensor de ordem $r+1$, com componentes, no referencial $\left\{e_{i}\right\}$ :

$$
F_{i_{1} i_{2} \ldots i_{r} ; j}=\nabla F\left(e_{i_{1}}, \ldots, e_{i_{r}} ; e_{j}\right)
$$

Essa definição é independente da escolha do referencial e segue a seguinte relação: 


$$
\sum_{j} F_{i_{1} \ldots i_{r}, j} \omega_{j}=d F_{i_{1} \ldots i_{r}}+\sum_{j} F_{j i_{2} i_{3} \ldots i_{r}} \omega_{j i_{1}}+\sum_{j} F_{i_{1} j i_{3} \ldots i_{r}} \omega_{j i_{2}}+\ldots+\sum_{j} F_{i_{1} \ldots i_{r-1} j} \omega_{j i_{r}} .
$$

Sendo assim, temos em particular, que as coordenadas $h_{i j k}$ da diferencial covariante respeitam:

$$
\sum_{k} h_{i j k} \omega_{k}=d h_{i j}+\sum_{k} h_{k j} \omega_{k i}+\sum_{k} h_{i k} \omega_{k j}
$$

Mas como a segunda forma fundamental é uma aplicação bilinear simétrica, então sua matriz é igual a sua transposta, com isso temos que $h_{i j}=h_{j i}, \forall i, j$, logo:

$$
\sum_{k} h_{i j k} \omega_{k}=d h_{i j}+\sum_{k} h_{i k} \omega_{k j}+\sum_{k} h_{j k} \omega_{k i}
$$

\section{Equação de Codazzi}

Com o objetivo de demonstrarmos que $h_{i j k}=h_{i k j}$, na expressão acima vamos tomar o produto exterior com $\omega_{j}$ e vamos somar em $j$ :

$$
\sum_{k j} h_{i j k} \omega_{k} \wedge \omega_{j}=\sum_{j} d h_{i j} \wedge \omega_{j}+\sum_{m j} h_{i m} \omega_{m j} \wedge \omega_{j}+\sum_{m j} h_{m j} \omega_{m i} \wedge \omega_{j}
$$

Note que:

$$
\sum_{m j} h_{m j} \omega_{m i} \wedge \omega_{j}=\sum_{m} \omega_{m i} \wedge\left(\sum_{j} h_{m j} \omega_{j}\right),
$$

e pelo Lema de Cartan:

$$
\omega_{n+1, i}=\sum_{j} h_{i j} \omega_{j}
$$

Logo:

$$
\sum_{m} \omega_{m i} \wedge\left(\sum_{j} h_{m j} \omega_{j}\right)=\sum_{m} \omega_{i m} \wedge \omega_{m, n+1} .
$$

Por (2.5), temos que $\Omega_{n+1 i}=0$ quando restrita a $M^{n}$. Logo, das equações de estrutura temos:

$$
\begin{aligned}
d \omega_{n+1, i} & =\sum_{m} \omega_{n+1, m} \wedge \omega_{m i}+\Omega_{n+1 i} \\
& =\sum_{m} \omega_{n+1, m} \wedge \omega_{m i} \\
& =-\sum_{m} \omega_{i m} \wedge \omega_{m, n+1} .
\end{aligned}
$$

Agora, derivando (2.7) exteriormente, usando (2.6), (2.8) e (2.3) temos: 


$$
\begin{aligned}
-\sum_{m} \omega_{i m} \wedge \omega_{m, n+1}= & d \omega_{n+1, i} \\
= & \sum_{j} h_{i j} d \omega_{j}+\sum_{j} d\left(h_{i j}\right) \wedge \omega_{j} \\
= & \sum_{j m} h_{i j} \omega_{j m} \wedge \omega_{m}-\sum_{j m} h_{i m} \omega_{m j} \wedge \omega_{j}-\sum_{m j} h_{m j} \omega_{m i} \wedge \omega_{j} \\
& +\sum_{k j} h_{i j k} \omega_{k} \wedge \omega_{j} \\
= & \sum_{j m} h_{i j} \omega_{j m} \wedge \omega_{m}-\sum_{j m} h_{i m} \omega_{m j} \wedge \omega_{j}-\sum_{m} \omega_{i m} \wedge \omega_{m, n+1} \\
& +\sum_{k j} h_{i j k} \omega_{k} \wedge \omega_{j} .
\end{aligned}
$$

Veja que, fazendo uma reordenação de índices, temos que

$$
\sum_{j m} h_{i j} \omega_{j m} \wedge \omega_{m}-\sum_{j m} h_{i m} \omega_{m j} \wedge \omega_{j}=\sum_{j l} h_{i j} \omega_{j l} \wedge \omega_{l}-\sum_{l m} h_{i m} \omega_{m l} \wedge \omega_{l}
$$

onde $m=l$ na primeira parcela e $j=l$ na segunda parcela.

Mas note que, fazendo $j=t$ na primeira parcela e $m=t$ na segunda, temos:

$$
+\sum_{j l} h_{i j} \omega_{j l} \wedge \omega_{l}-\sum_{l m} h_{i m} \omega_{m l} \wedge \omega_{l}=+\sum_{t l} h_{i t} \omega_{t l} \wedge \omega_{l}-\sum_{l t} h_{i t} \omega_{t l} \wedge \omega_{l}
$$

e com isso, concluímos que a expressão é nula!

Portanto:

$$
\begin{aligned}
& -\sum_{m} \omega_{i m} \wedge \omega_{m, n+1}=-\sum_{m} \omega_{i m} \wedge \omega_{m, n+1}+\sum_{k j} h_{i j k} \omega_{k} \wedge \omega_{j} . \\
& \Longrightarrow \sum_{k j} h_{i j k} \omega_{k} \wedge \omega_{j}=0 .
\end{aligned}
$$

Logo

$$
h_{i j k}=h_{i k j}
$$

Analogamente, para a segunda derivada temos:

$$
\sum_{l} h_{i j k l} \omega_{l}=d h_{i j k}+\sum_{l} h_{l j k} \omega_{l i}+\sum_{l} h_{i l k} \omega_{l j}+\sum_{k} h_{i j l} \omega_{l k} .
$$

Para uso nos próximos desenvolvimentos, faremos o convenção dos índices:

$$
\sum_{l} h_{i j k l} \omega_{l}=d h_{i j k}+\sum_{m} h_{m j k} \omega_{m i}+\sum_{m} h_{i m k} \omega_{m j}+\sum_{m} h_{i j m} \omega_{m k} .
$$

Agora, tomano o produto exterior com $\omega_{k}$ e somando em $k$, teremos: 
$\sum_{k l} h_{i j k l} \omega_{l} \wedge \omega_{k}=\sum_{k} d h_{i j k} \wedge \omega_{k}+\sum_{k m} \omega_{m i} \wedge_{k}+\sum_{k m} h_{i m k} \omega_{m j} \wedge \omega_{k}+\sum_{k m} h_{i j m} \omega_{m k} \wedge \omega_{k}$

Derivando exteriormente (2.6) temos:

$$
\begin{aligned}
\sum_{k} d h_{i j k} \wedge \omega_{k}+\sum_{k} h_{i j k} d \omega_{k}= & d^{2} \varkappa_{i j}+\sum_{m} d h_{i m} \wedge \omega_{m j}+\sum_{m} h_{i m} d \omega_{m j} \\
& +\sum_{m} d h_{m j} \wedge \omega_{m i}+\sum_{m} h_{m j} d \omega_{m i} .
\end{aligned}
$$

Usando agora (2.3) e (2.6), substituindo em (2.12) teremos:

$$
\begin{aligned}
\sum_{k} d h_{i j k} \wedge \omega_{k}+\sum_{k} h_{i j k} d \omega_{k} & =\sum_{m}\left(-\sum_{l} h_{i l} \omega_{l m}-\sum_{l} h_{m l} \omega_{l i}+\sum_{l} h_{i m l} \omega_{l}\right) \wedge \omega_{m j} \\
& +\sum_{m}\left(-\sum_{l} h_{m l} \omega_{l j}-\sum_{l} h_{j l} \omega_{l m}+\sum_{l} h_{m j l} \omega_{l}\right) \wedge \omega_{m i} \\
& +\sum_{m} h_{i m} d \omega_{m j}+\sum_{m} h_{m j} d \omega_{m i} \\
& =\sum_{m l} h_{i m l} \omega_{l} \wedge \omega_{m j}-\sum_{m l} h_{i l} \omega_{l m} \wedge \omega_{m j}-\sum_{m l} h_{m l} \omega_{l i} \wedge \omega_{m j} \\
& +\sum_{m l} h_{m j l} \omega_{l} \wedge \omega_{m i}-\sum_{m l} h_{m l} \omega_{l j} \wedge \omega_{m i}+\sum_{m l} h_{j l} \omega_{l m} \wedge \omega_{m i} \\
& +\sum_{m l} h_{i m} \omega_{m l} \wedge \omega_{l j}-\frac{1}{2} \sum_{m l k} h_{i m} R_{m j k l} \omega_{k} \wedge \omega_{l}+\sum_{m l} h_{m j} \omega_{m l} \wedge \omega_{l i} \\
& -\frac{1}{2} \sum_{m l k} h_{m j} R_{m i k l} \omega_{k} \wedge \omega_{l} .
\end{aligned}
$$

Portanto:

$$
\begin{gathered}
\sum_{k} d h_{i j k} \wedge \omega_{k}+\sum_{k} h_{i j k} d \omega_{k}=-\frac{1}{2} \sum_{m l k} h_{m j} R_{m i k l} \omega_{k} \wedge \omega_{l}-\frac{1}{2} \sum_{m l k} h_{i m} R_{m j k l} \omega_{k} \wedge \omega_{l} \\
+\sum_{m l} h_{m j l} \omega_{l} \wedge \omega_{m i}+\sum_{m l} h_{i m l} \omega_{l} \wedge \omega_{m j} .
\end{gathered}
$$

Substituindo agora (2.11) em (2.3) temos:

$$
\begin{aligned}
\sum_{k l} h_{i j k l} \omega_{l} \wedge \omega_{k}= & -\frac{1}{2} \sum_{m k l} h_{i m} R_{m j k l} \omega_{k} \wedge \omega_{l}+\sum_{m l} \omega_{l} \wedge \omega_{m j}-\frac{1}{2} \sum_{m k l} h_{m j} R_{m i k l} \omega_{k} \wedge \omega_{l} \\
& +\sum_{m l} h_{m j l} \omega_{l} \wedge \omega_{m i}-\sum_{k l} h_{i j k} \omega_{k l} \wedge \omega_{l}+\sum_{k m} h_{m j k} \omega_{m i} \wedge \omega_{k} \\
& +\sum_{k m} h_{i m k} \omega_{m j} \wedge \omega_{k}+\sum_{k m} h_{i j m} \omega_{m k} \wedge \omega_{k} .
\end{aligned}
$$


Logo:

$$
\sum_{k l} h_{i j k l} \omega_{l} \wedge \omega_{k}=-\frac{1}{2} \sum_{m k l} h_{i m} R_{m j k l} \omega_{k} \wedge \omega_{l}-\frac{1}{2} \sum_{m k l} h_{m j} R_{m i k l} \omega_{k} \wedge \omega_{l} .
$$

Concluímos então que:

$$
\sum_{k l}\left(h_{i j k l}-\frac{1}{2} \sum_{m} h_{i m} R_{m j k l}-\frac{1}{2} h_{m j} R_{m i k l}\right) \omega_{k} \wedge \omega_{l}=0 .
$$

Com isso:

$$
h_{i j k l}=\frac{1}{2} \sum_{m} h_{i m} R_{m j k l}+\frac{1}{2} h_{m j} R_{m i k l} .
$$

O que nos leva à:

$$
h_{i j l k}=\frac{1}{2} \sum_{m} h_{i m} R_{m j l k}+\frac{1}{2} h_{m j} R_{m i l k} .
$$

Sendo assim, usando que $R_{i j k l}=-R_{i j l k}$, temos que:

$$
h_{i j k l}-h_{i j l k}=\sum_{m} h_{i m} R_{m j k l}+\sum_{m} h_{m j} R_{m i k l} .
$$

Definindo agora

$$
\Delta h_{i j}=\sum_{k} h_{i j k k}
$$

teremos, por (2.9), as seguintes relações:

$$
h_{i j k}=h_{k i j} \Rightarrow h_{i j k k}=h_{k i k j} .
$$

Com isso, somaremos zero na definição acima e teremos:

$$
\begin{aligned}
\Delta h_{i j} & =\sum_{k}\left(h_{k i k j}+h_{k i j k}-h_{k i k j}\right) \\
& =\sum_{k} h_{k i k j}+\sum_{k m} h_{k m} R_{m i j k}+\sum_{k m} h_{m i} R_{m k j k} .
\end{aligned}
$$

Mas note que:

$$
h_{k i k}=h_{k k i} \Rightarrow h_{k i k j}=h_{k k i j} .
$$

E assim temos:

$$
\Delta h_{i j}=\sum_{k} h_{k k i j}+\sum_{k m} h_{k m} R_{m i j k}+\sum_{k m} h_{m i} R_{m k j k} .
$$




\subsection{Curvatura de Ricci e Curvatura Escalar}

Na seção 4 de [14], é apresentada a expressão do tensor curvatura de Ricci em coordenadas de um referencial, $R_{i k}=\sum_{s j} R_{i j k s} g^{s j}$, onde $\left[g_{s j}\right]=<e_{s}, e_{j}>=\epsilon_{s} \delta_{s j}=\delta_{s j}$ e $\left[g^{s j}\right]$ é a matriz inversa correspondente.

Fazendo as contas, e usando (2.5), temos então que:

$$
\begin{aligned}
R_{i k} & =\sum_{s j}\left(\delta_{i s} \delta_{j k}-\delta_{i k} \delta_{j s}+h_{i s} h_{j k}-h_{i k} h_{j s}\right) g^{s j} \\
& =\sum_{s j} \delta_{i s} \delta_{j k} \delta_{s j}-\delta_{i k} \sum_{s j} \delta_{j s} \delta_{s j}+\sum_{s j} h_{i s} h_{j k} \delta_{s j}-h_{i k} \sum_{s j} h_{j s} \delta_{j s} \\
& =\delta_{i k}-\delta_{i k} n+\sum_{j} h_{i j} h_{j k}-h_{i k} \sum_{s} h_{s s} \\
& =\delta_{i k}-\delta_{i k} n+\sum_{j} h_{i j} h_{j k}-h_{i k} n H \\
& =-(n-1) \delta_{i k}-h_{i k} n H+\sum_{s} h_{i j} h_{j k} .
\end{aligned}
$$

Note que, no processo utilizamos que $n H=\sum_{s} h_{s s}$.

Logo,

$$
R_{i j}=-(n-1) \delta_{i j}-n H h_{i j}+\sum_{k} h_{i k} h_{k j}
$$

Tal equação é escrita de maneira geral da forma

$$
R_{i j}=c(n-1) \delta_{i j}-n H h_{i j}+\sum_{k} h_{i k} h_{k j}
$$

Para a obtenção da expressão da curvatura escalar em termos da curvatura de Ricci, é somente necessário um cálculo direto da relação:

$$
\begin{aligned}
R & =\frac{1}{n(n-1)} \sum_{i k} R_{i k} g^{i k} \\
& =\frac{1}{n(n-1)} \sum_{i} R_{i k} \delta_{i k} \\
& =\frac{1}{n(n-1)} \sum_{i} R_{i i} .
\end{aligned}
$$

Da igualdade anterior e de (2.16), é possível estabelecer uma relação entre $R$ e $S$, isto é, entre a curvatura escalar e a norma da segunda forma fundamental. De fato, observe que $n^{2} H=n \sum_{i} h_{i i}$, usando (2.16) temos: 


$$
\begin{aligned}
n(n-1)(R+1)= & n^{2} R+n^{2}-n R-n \\
= & \frac{n}{n-1} \sum_{i} R_{i i}+n^{2}-\frac{1}{n-1} \sum_{i} R_{i i}-n \\
= & \frac{n}{n-1} \sum_{i}\left[-(n-1) \delta_{i i}-n H h_{i i}+\sum_{k} h_{i k}^{2}\right]- \\
& \frac{1}{n-1} \sum_{i}\left[-(n-1) \delta_{i i}-n H h_{i i}+\sum_{k} h_{i k}^{2}\right]-n+n^{2} \\
= & \frac{n}{n-1}\left[\sum_{i}\left(-\delta_{i i} n\right)+\sum_{i} \delta_{i i}-\sum_{i} n H h_{i i}+\sum_{i, k} h_{i k}^{2}\right]- \\
= & \frac{1}{n-1}\left[\sum_{i}\left(-n \delta_{i i}\right)+\sum_{i} \delta_{i i}-\sum_{i} n H h_{i i}+\sum_{i, k} h_{i k}^{2}\right]-n+n^{2} \\
= & \frac{-n^{3}}{n-1}+\frac{n^{2}}{n-1}-\frac{n^{3} H^{2}}{n-1}+\frac{n S}{n-1}+\frac{n^{2}}{n-1}-\frac{n}{n-1}+\frac{n^{2} H^{2}}{n-1}-\frac{S}{n-1} \\
= & \frac{-n^{3}+n^{2}-n^{3} H^{2}+n S+n^{2}-n+n^{2} H^{2}-S-n^{2}+n+n^{3}-n^{2}}{n-1} \\
= & \frac{(n-1) S-n^{2} H^{2}(n-1)}{n-1} \\
= & S H^{2}, \\
= & \\
= & \\
= & \\
= &
\end{aligned}
$$

$\log 0$

$$
n(n-1)(R+1)=S-n^{2} H^{2}
$$

\subsection{Ferramentas técnicas}

Para uso futuro, faremos nesta seção o desenvolvimento de dois lemas técnicos e da equação denominada tipo Simons-Calabi, encontrada em [17].

Lema 2.5.1. Sejam $\lambda_{i}$ e $\beta_{i j}, i, j=1, \ldots, n$, números reais, tais que $\lambda_{n}<0<\lambda_{1} \leq \cdots \leq \lambda_{n-1}$ e $\sum_{i} \lambda_{i}=\sum_{i} \beta_{i j}=\sum_{i} \lambda_{i} \beta_{i j}=0, j=1, \ldots, n$.

Fixado $j \in\{1, \ldots, n\}$ seja $g_{j} \in\{1, \ldots, n\}$ o maior índice tal que $\left|\beta_{g_{j} j}\right|=\max \left\{\left|\beta_{i}\right| i=1, \ldots, n\right\}$. Então $g_{j}=n \Longleftrightarrow \beta_{i j}=0, \forall i \leq n$.

Demonstração. Seja $g_{j}=n$, suponha por absurdo que $\beta_{n j} \neq 0$ e, sem perda de generalidade, tomamos $\beta_{n j}>0$.

Por hipótese temos que 


$$
\begin{aligned}
0 & =\sum_{i} \lambda_{i} \\
& =\sum_{i} \beta_{i j} \\
& =\sum_{i} \lambda_{i} \beta_{i j} \\
& =\sum_{i=1}^{n-1} \lambda_{i} \beta_{i j}+\lambda_{n} \beta_{n j} \\
& =\sum_{i=1}^{n-1} \lambda_{i} \beta_{i j}+\left(-\sum_{i=1}^{n-1} \lambda_{i}\right) \beta_{n j} \\
& =\sum_{i=1}^{n-1} \lambda_{i}\left(\beta_{i j}-\beta_{n j}\right) .
\end{aligned}
$$

Donde segue que $\left(\beta_{i j}-\beta_{n j}\right) \leq 0$, pois $\lambda_{i}>0, i=1, \ldots, n-1$, e $\beta_{n j}=\max \left\{\left|\beta_{i j}\right| i=1, \ldots, n-1\right\}$ Logo $\beta_{i j}=\beta_{n j}, i=1, \ldots, n-1$.

Como $\sum_{i} \beta_{i j}=0$, e usando a conclusão acima, temos que

$$
\sum_{i}^{n} \beta_{n j}=0
$$

O que nos leva a concluir que $\beta_{n j}=0$, o que é uma contradição.

Reciprocamente, supondo que $\beta_{i j}=0$ para todo $i$, segue diretamente que $g_{j}=n$.

Lema 2.5.2. Sejam $A=\left[a_{i j}\right]$ e $B=\left[b_{i j}\right]$ matrizes $n \times n$, simétricas, ou seja, $a_{i j}=a_{j i}$ e $b_{i j}=b_{j i}$ para todos $i, j$. Então $\operatorname{tr}\left[(A B-B A)(A B-B A)^{t}\right] \geq 0$.

Demonstração. Para a demonstração do resultado, veremos que todo elemento da diagonal principal da matriz $(A B-B A)(A B-B A)^{t}$ será positivo, e com isso, segue o desejado.

Seja $C=A B=\left[c_{i j}\right]$, onde cada elemento da matriz $C$ tem a forma

$$
c_{i j}=\sum_{k}^{n} a_{i k} b_{k j} .
$$

Seja $D=B A=\left[d_{i j}\right]$, onde cada elemento da matriz $D$ tem a forma

$$
d_{i j}=\sum_{k}^{n} b_{i k} a_{k j} .
$$

Portanto, cada elemento da matriz $A B-B A=\left[c_{i j}-d_{i j}\right]$ tem a forma

$$
c_{i j}-d_{i j}=\sum_{k}^{n}\left(a_{i k} b_{k j}-b_{i k} a_{k j}\right) .
$$


Analogamente, cada elemento da matriz $(A B-B A)^{t}$ tem a forma

$$
c_{j i}-d_{j i}=\sum_{k}^{n}\left(a_{k i} b_{j k}-b_{k i} a_{j k}\right) .
$$

Definido a matriz $F=(A B-B A)(A B-B A)^{t}=\left[f_{i j}\right]$ temos que cada elemento $f_{i j}$ tem a forma

$$
\begin{aligned}
f_{i j} & =\sum_{l}\left(c_{i l}-d_{i l}\right)\left(c_{j l}-d_{j l}\right) \\
& =\sum_{l} \sum_{k}^{n}\left[\left(a_{i k} b_{k l}-b_{i k} a_{k l}\right)\left(a_{j k} b_{k l}-b_{j k} a_{k l}\right)\right] \\
& =\sum_{k}^{n}\left(a_{i k} b_{k l} a_{j k} b_{k l}-a_{i k} b_{k l} b_{j k} a_{k l}-b_{i k} a_{k l} a_{j k} b_{k l}+b_{i k} a_{k l} b_{j k} a_{k l}\right) .
\end{aligned}
$$

Logo, da definição da função traço, sabe-se que $\operatorname{tr}(F)=\sum_{i}^{n}=f_{i i}$, e por meio de cálculo direto temos

$$
\sum_{i} f_{i i}=\sum_{l}^{n} \sum_{k}^{n}\left[\left(a_{i k} b_{k l}-b_{i k} a_{k l}\right)\left(a_{i k} b_{k l}-b_{i k} a_{k l}\right)\right] .
$$

Assim, concluímos que

$$
\sum_{i} f_{i i}=\sum_{k l}^{n}\left(a_{i k} b_{k l}-b_{i k} a_{k l}\right)^{2} .
$$

Portanto, $\operatorname{tr}\left[(A B-B A)(A B-B A)^{t}\right] \geq 0$.

Agora reescreveremos algumas equações já desenvolvidas na seção 2.3, para o caso de codimensão arbitrária igual a $p$, isto é, no caso que $M^{n}$ é uma subvariedade imersa no espaço pseudo-riemanniano com curvatura constante $c$ denotado por $L_{p}^{n+p}(c),(c<0)$.

Para fixar a notação, adicionaremos o índice da codimensão a frente dos demais índices utilizados, com isso, $h_{\alpha i j}$ denotará a componente da segunda forma fundamental, na direção normal $\alpha$. Usamos ainda a seguinte convenção

$$
n+1 \leq \alpha, \beta, \cdots \leq n+p
$$

A equação de Gauss (2.5), para o caso de codimensões superiores tem a forma

$$
R_{i j k l}=c\left(\delta_{i k} \delta_{j l}-\delta_{i l} \delta_{j k}\right)-\sum_{\alpha} h_{\alpha i k} h_{\alpha j l}-h_{\alpha i l} h_{\alpha j k}
$$

onde $c$ é o valor da curvatura seccional de $L_{p}^{n+p}(c)$.

A equação da curvatura de Ricci, onde os coeficientes estão no fibrado normal de $M$, será

$$
R_{\alpha \beta i j}=\sum_{m}\left(h_{\alpha i m} h_{\beta m j}-h_{\alpha j m} h_{\beta i m}\right) .
$$


A equação que nos dá a relação entre a derivada covariante de $h_{\alpha i j}$ e $d h_{\alpha i j}$, como descrita em (2.6), no caso de codimensões maiores que 1 fica

$$
\sum_{k} h_{\alpha i j k} \omega_{k}=d h_{\alpha i j}+\sum_{k} h_{\alpha i k} \omega_{k j}+\sum_{k} h_{\alpha j k} \omega_{k i}-\sum_{\beta} h_{\beta i j} \omega_{\beta \alpha}
$$

Analogamente, para a segunda derivada, a equação (2.10) fica

$$
\sum_{l} h_{\alpha i j k l} \omega_{l}=d h_{\alpha i j k}+\sum_{l} h_{\alpha l j k} \omega_{l i}+\sum_{l} h_{\alpha i l k} \omega_{l j}+\sum_{l} h_{\alpha i j l} \omega_{l k}-\sum_{\beta} h_{\beta i j k} \omega_{\beta \alpha} .
$$

Seguindo de forma análoga ao desenvolvimento de (2.13), temos para codimensão arbitrária que

$$
h_{\alpha i j k l}-h_{\alpha i j l k}=\sum_{m} h_{\alpha i m} R_{m j k l}+\sum_{m} h_{\alpha m j} R_{m i k l}+\sum_{\beta} h_{\beta i j} R_{\alpha \beta k l} .
$$

Já a equação do laplaciano descrita em (2.15),para o caso de $h_{\alpha i j}$ fica

$$
\Delta h_{\alpha i j}=\sum_{k} h_{\alpha k k i j}+\sum_{k m} h_{\alpha k m} R_{m i j k}+\sum_{k m} h_{\alpha m i} R_{m k j k}+\sum_{k \beta} h_{\beta i k} R_{\alpha \beta j k} .
$$

Agora podemos mostrar como a equação tipo Simons-Calabi pode ser deduzida, a partir das igualdades acima.

Proposição 2.5.3 (Equação tipo Simons-Calabi). Seja $M$ uma subvariedade de dimensão n, imersa em $L_{p}^{n+p}(c)$ de dimensão $n+p$. Se $M$ é maximal em $N$, então

$$
\frac{1}{2} \Delta S=\sum\left(h_{\alpha i j k}\right)^{2}+n c S+\frac{1}{p} S^{2}+\frac{1}{p} \sum_{\alpha<\beta}\left(S_{\alpha}-S_{\beta}\right)^{2}+\sum_{\alpha \beta} N\left(H_{\alpha} H_{\beta}-H_{\beta} H_{\alpha}\right) .
$$

Onde $S_{\alpha \beta}=\sum_{i j} h_{\alpha i j} h_{\beta i j}, S_{\alpha \alpha}=S_{\alpha}, H_{\alpha}$ é a matriz simétrica dos $\left(h_{\alpha i j}\right)$ e $N(A)=\operatorname{tr}\left(A . A^{t}\right)$, onde tr é o operador traço.

Demonstração. Seja $\left\{e_{1}, e_{2}, \ldots, e_{n+p}\right\}$ um referencial ortonormal local, pseudo-riemanniano em $L^{n+p}(c)$, de tal modo que $\left\{e_{1}, \ldots, e_{n}\right\}$ seja uma base para o $T_{p} M$. Tal referencial é dito adaptado a $M$.

Devido a linearidade do operador laplaciano, temos que

$$
\Delta S=\Delta\left(\sum_{\alpha i j}\left(h_{\alpha i j}\right)^{2}\right)=\sum_{\alpha i j} \Delta\left(h_{\alpha i j}\right)^{2} .
$$

Note que, a $k$-ésima derivada parcial de $h_{\alpha i j}$, tem a seguinte expressão

$$
\left[\left(h_{\alpha i j}\right)^{2}\right]_{k}=2 h_{\alpha i j} h_{\alpha i j k} .
$$

Como o laplaciano envolve a soma das segundas derivadas parciais da função em questão, vamos calcular a segunda derivada $k$-ésima de $h_{\alpha i j}$, portanto, usando a regra da cadeia temos 


$$
\begin{aligned}
{\left[\left(h_{\alpha i j}\right)^{2}\right]_{k k} } & =\left[2 h_{\alpha i j} h_{\alpha i j k}\right]_{k} \\
& =2\left[h_{\alpha i j k} h_{\alpha i j k}+h_{\alpha i j} h_{\alpha i j k k}\right] \\
& =2\left[\left(h_{\alpha i j k}\right)^{2}+h_{\alpha i j} h_{\alpha i j k k}\right] .
\end{aligned}
$$

Portanto, para obtermos o laplaciano de cada $\left(h_{\alpha i j}\right)^{2}$, basta fazermos a soma em $k$, então

$$
\Delta\left(h_{\alpha i j}\right)^{2}=\sum_{k} 2\left[\left(h_{\alpha i j k}\right)^{2}+h_{\alpha i j} h_{\alpha i j k k}\right]
$$

Substituindo a igualdade acima em (2.27), temos

$$
\Delta S=\sum_{\alpha i j k}\left(2\left[\left(h_{\alpha i j k}\right)^{2}+h_{\alpha i j} h_{\alpha i j k k}\right]\right),
$$

e usando que $\Delta h_{\alpha i j}=\sum_{k} h_{\alpha i j k k}$, temos que

$$
\frac{1}{2} \Delta S=\sum_{\alpha i j k}\left(h_{\alpha i j k}\right)^{2}+\sum_{\alpha i j} h_{\alpha i j} \Delta h_{\alpha i j} .
$$

Note que o primeiro membro da equação tipo Simons-Calabi já está presente na equação acima, portanto resta apenas os outros somandos. Para isso, substituiremos a equação (2.25) na igualdade acima, obtendo

$$
\begin{aligned}
\frac{1}{2} \Delta S= & \sum_{\alpha i j k}\left(h_{\alpha i j k}\right)^{2}+\sum_{\alpha i j} h_{\alpha i j}\left(\sum_{k} h_{\alpha k k i j}+\sum_{k m} h_{\alpha k m} R_{m i j k}\right. \\
& \left.+\sum_{k m} h_{\alpha m i} R_{m k j k}+\sum_{k \beta} h_{\beta i k} R_{\alpha \beta j k}\right) \\
= & \sum_{\alpha i j k}\left(h_{\alpha i j k}\right)^{2}+\sum_{\alpha i j} h_{\alpha i j}\left(\sum_{k} h_{\alpha k k i j}\right)+\sum_{\alpha i j} h_{\alpha i j}\left(\sum_{m k} h_{\alpha k m} R_{m i j k}\right) \\
& +\sum_{\alpha i j}\left(\sum_{m k} h_{\alpha i j} h_{\alpha m i} R_{m k j k}\right)+\sum_{\alpha i j} h_{\alpha i j}\left(\sum_{k \beta} h_{\beta i k} R_{\alpha \beta j k}\right) \\
= & \sum_{\alpha i j k}\left(h_{\alpha i j k}\right)^{2}+\sum_{\alpha i j k} h_{\alpha i j} h_{\alpha k k i j}+\sum_{\alpha i j k m} h_{\alpha i j} h_{\alpha k m} R_{m i j k} \\
& +\sum_{\alpha i j k m} h_{\alpha i j} h_{\alpha m i} R_{m k j k}+\sum_{\alpha \beta i j k} h_{\alpha i j} h_{\beta i k} R_{\alpha \beta j k} .
\end{aligned}
$$

Após isso, vale notar que, como $M$ é por hipótese, maximal, temos

$$
\sum_{\alpha i j k} h_{\alpha i j} h_{\alpha k k i j}=0
$$

pois

$$
\sum_{k} h_{\alpha k k}=0
$$

para todo $\alpha$ fixado. Agora basta substituirmos as expressões de $R_{m i j k}, R_{m k j k}$ e $R_{\alpha \beta j k}$. Para um melhor entendimento, vamos analisar cada parcela separadamente. 
- Desenvolvimento de $\sum_{\alpha i j k m} h_{\alpha i j} h_{\alpha k m} R_{m i j k}$ :

Substituindo o valor de $R_{m i j k}$ na expressão acima temos

$$
\begin{aligned}
\sum_{\alpha i j k m} h_{\alpha i j} h_{\alpha k m} R_{m i j k}= & \sum_{\alpha i j k m} h_{\alpha i j} h_{\alpha k m}\left(c\left(\delta_{m j} \delta_{i k}-\delta_{m k} \delta_{i j}\right)-\sum_{\beta}\left(h_{\beta m j} h_{\beta i k}-h_{\beta m k} h_{\beta i j}\right)\right) \\
= & \sum_{\alpha i j k m} h_{\alpha i j} h_{\alpha k m} c\left(\delta_{m j} \delta_{i k}-\delta_{m k} \delta_{i j}-\sum_{\alpha \beta i j k m} h_{\alpha i j} h_{\alpha k m}\left(h_{\beta m j} h_{\beta i k}-h_{\beta m k} h_{\beta i j}\right)\right. \\
= & c \sum_{\alpha i m} h_{\alpha i m} h_{\alpha i m}-c \sum_{\alpha i m} h_{\alpha i i} h_{\alpha m m}+\sum_{\alpha \beta i j k m} h_{\alpha i j} h_{\alpha k m} h_{\beta m k} h_{\beta i j} \\
& -\sum_{\alpha \beta i j k m} h_{\alpha i j} h_{\alpha k m} h_{\beta m j} h_{\beta i k},
\end{aligned}
$$

e usando que $M$ é maximal e $S=\sum_{\alpha i j} h_{\alpha i j}^{2}$ temos

$$
\sum_{\alpha i j k m} h_{\alpha i j} h_{\alpha k m} R_{m i j k}=c S+\sum_{\alpha \beta i j k m} h_{\alpha i j} h_{\alpha k m} h_{\beta m k} h_{\beta i j}-\sum_{\alpha \beta i j k m} h_{\alpha i j} h_{\alpha k m} h_{\beta m j} h_{\beta i k} .
$$

Passamos agora para a próxima parcela.

- Desenvolvimento do termo $\sum_{\alpha i j k m} h_{\alpha i j} h_{\alpha m i} R_{m k j k}$ :

Substituindo o valor de $R_{m k j k}$ temos

$$
\begin{aligned}
\sum_{\alpha i j k m} h_{\alpha i j} h_{\alpha m i} R_{m k j k} & =\sum_{\alpha i j k m} h_{\alpha i j} h_{\alpha m i}\left(c\left(\delta_{m j} \delta_{k k}-\delta_{m k} \delta_{k j}\right)-\sum_{\beta}\left(h_{\beta m j} h_{\beta k k}-h_{\beta m k} h_{\beta k j}\right)\right) \\
& =c n \sum_{\alpha i j}\left(h_{\alpha i j}\right)^{2}-c \sum_{\alpha i j}\left(h_{\alpha i j}\right)^{2}-\sum_{\alpha \beta i j k m} h_{\alpha i j} h_{\alpha m i}\left(h_{\beta m j} h_{\beta k k}-h_{\beta m k} h_{\beta k j}\right. \\
& =c n S-c S+\sum_{\alpha \beta i j k m} h_{\alpha i j} h_{\alpha m i} h_{\beta m k} h_{\beta k j}-\sum_{\alpha \beta i j k m} h_{\alpha i j} h_{\alpha m i} h_{\beta m j} h_{\beta k k},
\end{aligned}
$$

e novamente usando o fato de $M$ ser maximal temos

$$
\sum_{\alpha i j k m} h_{\alpha i j} h_{\alpha m i} R_{m k j k}=c n S-c S+\sum_{\alpha \beta i j k m} h_{\alpha i j} h_{\alpha m i} h_{\beta m k} h_{\beta k j} .
$$

Vejamos agora o último termo.

- Análise do termo $\sum_{\alpha \beta i j k} h_{\alpha i j} h_{\beta i k} R_{\alpha \beta j k}$ :

Substituindo o valor de $R_{\alpha \beta j k}$ temos 


$$
\begin{aligned}
\sum_{\alpha \beta i j k} h_{\alpha i j} h_{\beta i k} R_{\alpha \beta j k} & =\sum_{\alpha \beta i j k} h_{\alpha i j} h_{\beta i k}\left(\sum_{m}\left(h_{\alpha j m} h_{\beta m k}-h_{\alpha k m} h_{\beta m j}\right)\right) \\
& =\sum_{\alpha \beta i j k m} h_{\alpha i j} h_{\beta i k} h_{\alpha j m} h_{\beta m k}-\sum_{\alpha \beta i j k m} h_{\alpha i j} h_{\beta i k} h_{\alpha k m} h_{\beta m j} .
\end{aligned}
$$

Ficamos então com

$$
\sum_{\alpha \beta i j k} h_{\alpha i j} h_{\beta i k} R_{\alpha \beta j k}=\sum_{\alpha \beta i j k m} h_{\alpha i j} h_{\beta i k} h_{\alpha j m} h_{\beta m k}-\sum_{\alpha \beta i j k m} h_{\alpha i j} h_{\beta i k} h_{\alpha k m} h_{\beta m j} .
$$

Somando (2.29), (2.30) e (2.31), temos

$$
\begin{gathered}
\sum_{\alpha i j k m} h_{\alpha i j} h_{\alpha k m} R_{m i j k}+\sum_{\alpha i j k m} h_{\alpha i j} h_{\alpha m i} R_{m k j k}+\sum_{\alpha \beta i j k} h_{\alpha i j} h_{\beta i k} R_{\alpha \beta j k}= \\
\sum_{\alpha \beta i j k m} h_{\alpha i j} h_{\alpha k m} h_{\beta m k} h_{\beta i j}-\sum_{\alpha \beta i j k m} h_{\alpha i j} h_{\alpha k m} h_{\beta m j} h_{\beta i k}+c n S+ \\
\sum_{\alpha \beta i j k m} h_{\alpha i j} h_{\alpha m i} h_{\beta m k} h_{\beta k j}+\sum_{\alpha \beta i j k m} h_{\alpha i j} h_{\beta i k} h_{\alpha j m} h_{\beta m k}-\sum_{\alpha \beta i j k m} h_{\alpha i j} h_{\beta i k} h_{\alpha k m} h_{\beta m j} .
\end{gathered}
$$

A partir deste ponto, três igualdades serão demonstradas, envolvendo as matrizes $H_{\alpha}$ e $H_{\beta}$, definidas anteriormente.

1. $\sum_{\alpha \beta i j k m} h_{\alpha i j} h_{\alpha k m} h_{\beta m j} h_{\beta i k}=\sum_{\alpha \beta} \operatorname{tr}\left(H_{\alpha} H_{\beta}\right)^{2}$.

Aqui, denotaremos por $\left(H_{\alpha} H_{\beta}\right)_{i j}$ o elemento $i j$ da matriz $\left(H_{\alpha} H_{\beta}\right)$.

Fazendo o cálculo direto do elemento $\left(H_{\alpha} H_{\beta}\right)$ temos

$$
\begin{aligned}
\left(H_{\alpha} H_{\beta}\right)_{i j}^{2} & =\sum_{m}\left(H_{\alpha} H_{\beta}\right)_{i m}\left(H_{\alpha} H_{\beta}\right)_{m j} \\
& =\sum_{m}\left(\sum_{k} h_{\alpha i k} h_{\beta k m}\right)\left(\sum_{r} h_{\alpha m r} h_{\beta r j}\right) \\
& =\sum_{m k r} h_{\alpha i k} h_{\beta k m} h_{\alpha m r} h_{\beta r j} .
\end{aligned}
$$

Calculando o traço teremos 


$$
\begin{aligned}
\operatorname{tr}\left(H_{\alpha} H_{\beta}\right)^{2} & =\sum_{i}\left(H_{\alpha} H_{\beta}\right)_{i i}^{2} \\
& =\sum_{i}\left(\sum_{m k r} h_{\alpha i k} h_{\beta k m} h_{\alpha m r} h_{\beta r i}\right) \\
& =\sum_{i m k r} h_{\alpha i k} h_{\beta k m} h_{\alpha m r} h_{\beta r i} .
\end{aligned}
$$

Agora, usando que as matrizes $H_{\alpha}$ e $H_{\beta}$ são simétricas, e substituindo os índices $k$ por $j$ e $r$ por $k$ teremos

$$
\begin{aligned}
\operatorname{tr}\left(H_{\alpha} H_{\beta}\right)^{2} & =\sum_{i m j k} h_{\alpha i j} h_{\beta j m} h_{\alpha m k} h_{\beta i k} \\
& =\sum_{i m j k} h_{\alpha i j} h_{\alpha m k} h_{\beta j m} h_{\beta i k} \\
& =\sum_{i m j k} h_{\alpha i j} h_{\alpha k m} h_{\beta m j} h_{\beta i k} .
\end{aligned}
$$

Portanto, obtemos que

$$
\sum_{\alpha \beta} \operatorname{tr}\left(H_{\alpha} H_{\beta}\right)^{2}=\sum_{\alpha \beta i m j k} h_{\alpha i j} h_{\alpha k m} h_{\beta m j} h_{\beta i k} .
$$

2. $\sum_{\alpha \beta i j k m} h_{\alpha i j} h_{\alpha m i} h_{\beta m k} h_{\beta k j}=\sum_{\alpha \beta} \operatorname{tr}\left(H_{\alpha} H_{\beta} H_{\beta} H_{\alpha}\right)$.

De maneira análoga, vemos que um elemento da matriz

$$
\left(H_{\alpha} H_{\beta}\right)_{i j}=\sum_{k} h_{\alpha i k} h_{\beta k j},
$$

e

$$
\left(H_{\beta} H_{\alpha}\right)_{i j}=\sum_{m} h_{\beta i m} h_{\alpha m j}
$$

e usando tal fato temos

$$
\begin{aligned}
\left(H_{\alpha} H_{\beta} H_{\beta} H_{\alpha}\right)_{i j} & =\sum_{r}\left(H_{\alpha} H_{\beta}\right)_{i r}\left(H_{\beta} H_{\alpha}\right)_{r j} \\
& =\sum_{r}\left(\sum_{k} h_{\alpha i k} h_{\beta k r}\right)\left(\sum_{m} h_{\alpha r m} h_{\beta m j}\right) \\
& =\sum_{r k m} h_{\alpha i k} h_{\beta k r} h_{\alpha r m} h_{\beta m j} .
\end{aligned}
$$

Agora usando a definição da função traço, temos 


$$
\operatorname{tr}\left(H_{\alpha} H_{\beta} H_{\beta} H_{\alpha}\right)=\sum_{i}\left(H_{\alpha} H_{\beta} H_{\beta} H_{\alpha}\right)_{i i}=\sum_{i r k m} h_{\alpha i k} h_{\beta k r} h_{\beta r m} h_{\alpha m i} .
$$

Então basta somarmos em $\alpha$ e $\beta$, usarmos a simetria das matrizes $H_{\alpha}$ e $H_{\beta}$ e trocando $r$ por $k$ e $k$ por $j$ no último passo teremos

$$
\begin{aligned}
\sum_{\alpha \beta} \operatorname{tr}\left(H_{\alpha} H_{\beta} H_{\beta} H_{\alpha}\right) & =\sum_{\alpha \beta i r k m} h_{\alpha i k} h_{\beta k r} h_{\beta r m} h_{\alpha m i} \\
& =\sum_{\alpha \beta i r k m} h_{\alpha i k} h_{\alpha m i} h_{\beta r m} h_{\beta k r} \\
& =\sum_{\alpha \beta i r k m} h_{\alpha i k} h_{\alpha m i} h_{\beta m r} h_{\beta r k} \\
& =\sum_{\alpha \beta i k j m} h_{\alpha i j} h_{\alpha m i} h_{\beta m k} h_{\beta k j} .
\end{aligned}
$$

Assim concluímos que

$$
\sum_{\alpha \beta} \operatorname{tr}\left(H_{\alpha} H_{\beta} H_{\beta} H_{\alpha}\right)=\sum_{\alpha \beta i k j m} h_{\alpha i j} h_{\alpha m i} h_{\beta m k} h_{\beta k j} .
$$

A igualdade seguinte é obtida sem o uso das coordenadas explicitamente, apenas utilizando as propriedades de matriz transposta e a linearidade da função traço.

3. $\sum_{\alpha \beta} N\left(H_{\alpha} H_{\beta}-H_{\beta} H_{\alpha}\right)=2 \sum_{\alpha \beta} \operatorname{tr}\left(H_{\alpha} H_{\beta} H_{\alpha} H_{\beta}\right)-2 \sum_{\alpha \beta} \operatorname{tr}\left(H_{\beta} H_{\alpha}\right)^{2}$.

Através de cálculo direto temos

$$
\begin{aligned}
\sum_{\alpha \beta} N\left(H_{\alpha} H_{\beta}-H_{\beta} H_{\alpha}\right)= & \sum_{\alpha \beta} \operatorname{tr}\left(\left(H_{\alpha} H_{\beta}-H_{\beta} H_{\alpha}\right)\left(H_{\alpha} H_{\beta}-H_{\beta} H_{\alpha}\right)^{t}\right) \\
= & \sum_{\alpha \beta} \operatorname{tr}\left(\left(H_{\alpha} H_{\beta}-H_{\beta} H_{\alpha}\right)\left(\left(H_{\alpha} H_{\beta}\right)^{t}-\left(H_{\beta} H_{\alpha}\right)^{t}\right)\right) \\
= & \sum_{\alpha \beta} \operatorname{tr}\left(\left(H_{\alpha} H_{\beta}-H_{\beta} H_{\alpha}\right)\left(\left(H_{\beta}\right)^{t}\left(H_{\alpha}\right)^{t}-\left(H_{\alpha}\right)^{t}\left(H_{\beta}\right)^{t}\right)\right) \\
= & \sum_{\alpha \beta} \operatorname{tr}\left(\left(H_{\alpha} H_{\beta}-H_{\beta} H_{\alpha}\right)\left(H_{\beta} H_{\alpha}-H_{\alpha} H \beta\right)\right) \\
= & \sum_{\alpha \beta} \operatorname{tr}\left(H_{\alpha} H_{\beta} H_{\beta} H_{\alpha}-\left(H_{\beta} H_{\alpha}\right)^{2}-\left(H_{\alpha} H_{\beta}\right)^{2}+H_{\beta} H_{\alpha} H_{\alpha} H_{\beta}\right) \\
= & \sum_{\alpha \beta} \operatorname{tr}\left(H_{\alpha} H_{\beta} H_{\beta} H_{\alpha}\right)-\sum_{\alpha \beta} \operatorname{tr}\left(H_{\beta} H_{\alpha}\right)^{2} \\
& -\sum_{\alpha \beta} \operatorname{tr}\left(H_{\alpha} H_{\beta}\right)^{2}+\sum_{\alpha \beta} \operatorname{tr}\left(H_{\beta} H_{\alpha} H_{\alpha} H_{\beta}\right) \\
= & 2 \sum_{\alpha \beta} \operatorname{tr}\left(H_{\alpha} H_{\beta} H_{\beta} H_{\alpha}\right)-2 \sum_{\alpha \beta} \operatorname{tr}\left(H_{\beta} H_{\alpha}\right)^{2} .
\end{aligned}
$$


Porntanto segue que

$$
\sum_{\alpha \beta} N\left(H_{\alpha} H_{\beta}-H_{\beta} H_{\alpha}\right)=2 \sum_{\alpha \beta} \operatorname{tr}\left(H_{\alpha} H_{\beta} H_{\beta} H_{\alpha}\right)-2 \sum_{\alpha \beta} \operatorname{tr}\left(H_{\beta} H_{\alpha}\right)^{2}
$$

Feito isso, substituindo (2.33), (2.34) e (2.35) em (2.32) temos

$$
\begin{gathered}
\sum_{\alpha i j k m} h_{\alpha i j} h_{\alpha k m} R_{m i j k}+\sum_{\alpha i j k m} h_{\alpha i j} h_{\alpha m i} R_{m k j k}+\sum_{\alpha \beta i j k} h_{\alpha i j} h_{\beta i k} R_{\alpha \beta j k}= \\
\sum_{\alpha \beta i j k m} h_{\alpha i j} h_{\alpha k m} h_{\beta m k} h_{\beta i j}+c n S-2 \sum_{\alpha \beta} \operatorname{tr}\left(H_{\alpha} H_{\beta}\right)^{2}+2 \sum_{\alpha \beta} \operatorname{tr}\left(H_{\alpha} H_{\beta} H_{\beta} H_{\alpha}\right)= \\
\sum_{\alpha \beta i j k m} h_{\alpha i j} h_{\alpha k m} h_{\beta m k} h_{\beta i j}+c n S+\sum_{\alpha \beta} N\left(H_{\alpha} H_{\beta}-H_{\beta} H_{\alpha}\right) .
\end{gathered}
$$

Agora notemos que, a norma da segunda forma fundamental pode ser reescrita, em termos de $S_{\alpha \beta}$ como

$$
S=\sum_{\alpha} S_{\alpha}
$$

e mais, a $p \times p$ matriz cujo cada elemento é igual a $S_{\alpha \beta}$ é simétrica devido a sua definição, e com isso podemos assumir que existe uma base $\left\{e_{n+1}, \ldots, e_{n+p}\right\}$ tal que ela seja diagonal. Com isso, temos

$$
\sum_{\alpha \beta i j k m} h_{\alpha i j} h_{\alpha k m} h_{\beta m k} h_{\beta i j}=\sum_{\alpha} S_{\alpha}^{2} .
$$

Então substituindo (2.36) e (2.37) em (2.28)

$$
\frac{1}{2} \Delta S=\sum_{\alpha i j k}\left(h_{\alpha i j k}\right)^{2}+n c S+\sum_{\alpha \beta} N\left(H_{\alpha} H_{\beta}-H_{\beta} H_{\alpha}\right)+\sum_{\alpha} S_{\alpha}^{2} .
$$

Agora, sejam $\sigma_{1}, \sigma_{2} \in \mathbb{R}$ tais que

$$
p \sigma_{1}=\sum_{\alpha} S_{\alpha}=S, \quad \frac{p(p-1)}{2} \sigma_{2}=\sum_{\alpha<\beta} S_{\alpha} S_{\beta} .
$$

Temos então que

$$
\begin{aligned}
\sum_{\alpha} S_{\alpha}^{2} & =\left(\sum_{\alpha} S_{\alpha}\right)^{2}-2 \sum_{\alpha<\beta} S_{\alpha} S_{\beta} \\
& =p^{2} \sigma_{1}^{2}-p(p-1) \sigma_{2}
\end{aligned}
$$

que pode ser reescrita como

$$
p^{2} \sigma_{1}^{2}-p(p-1) \sigma_{2}=p \sigma_{1}^{2}+p(p-1)\left(\sigma_{1}^{2}-\sigma_{2}\right)
$$


Por outro lado temos também que

$$
p^{2}(p-1)\left(\sigma_{1}^{2}-\sigma_{2}\right)=\sum_{\alpha<\beta}\left(S_{\alpha}-S_{\beta}\right)^{2} .
$$

Com isso, juntando (2.39) e (2.40) temos que

$$
\sum_{\alpha} S_{\alpha}^{2}=\frac{1}{p} S^{2}+\frac{1}{p} \sum_{\alpha<\beta}\left(S_{\alpha}-S_{\beta}\right)^{2} .
$$

Portanto, substituindo (2.41) em (2.38), obtemos a expressão desejada

$$
\frac{1}{2} \Delta S=\sum\left(h_{\alpha i j k}\right)^{2}+n c S+\frac{1}{p} S^{2}+\frac{1}{p} \sum_{\alpha<\beta}\left(S_{\alpha}-S_{\beta}\right)^{2}+\sum_{\alpha \beta} N\left(H_{\alpha} H_{\beta}-H_{\beta} H_{\alpha}\right) .
$$




\section{Capítulo 3}

\section{Subvariedades tipo espaço maximais em um espaço pseudo-riemanniano de curvatura constante}

O artigo de Toru Ishihara [17] de 1988 foi um dos grandes avanços na teoria de classificações de subvariedades imersas em espaços de curvatura constante, e merece destaque neste trabalho, uma vez que é utilizado para obtenção dos principais resultados aqui demonstrados.

No artigo [17], são demonstrados os seguintes teoremas:

Teorema 3.0.1. Seja $M$ uma variedade Riemanniana n-dimensional completa isométricamente imersa em um espaço pseudo-Riemanniano $L_{p}^{n+p}(c), c \geq 0$. Se $M$ é maximal, então a imersão é totalmente geodésica e $M$ é um espaço de curvatura constante $c$.

Teorema 3.0.2. Seja $M$ uma variedade Riemanniana n-dimensional, completa, isométricamente imersa em um espaço pseudo-Riemanniano $L_{p}^{n+p}(-c)$, de curvatura constante $-c,(c>0)$. Se $M$ é maximal, então temos $0 \leq S \leq n p c$.

Teorema 3.0.3. As subvariedades $\mathbb{H}^{n_{1}}\left(\sqrt{\frac{n_{1}}{n}}\right) \times \cdots \times \mathbb{H}^{n_{p+1}}\left(\sqrt{\frac{n_{p+1}}{n}}\right)$ de $\mathbb{H}_{p}^{n+p}$, onde $n_{i}$ são inteiros positivos tais que $n_{1}+\ldots n_{p+1}=n$, são as únicas subvariedades tipo espaço, conexas, completas e maximais de dimensão n em $\mathbb{H}_{p}^{n+p}$, satisfazendo $S=n p$.

Uma das principais ferramentas utilizadas para obtenção de tais resultados, é o Principio do Máximo Generalizado, ver [27], e enunciaremos a seguir na forma de um lema.

Lema 3.0.4 (Yau). Seja $M^{n}$ uma variedade Riemanniana completa, com curvatura de Ricci limitada por baixo e seja $f: M^{n} \rightarrow \mathbb{R}$ uma função $C^{2}$ que é limitada por baixo em $M^{n}$. Então existe uma sequência de pontos $\left\{p_{k}\right\}$ em $M^{n}$ tais que:

$$
(i) F\left(p_{k}\right)<\inf F+\frac{1}{k}, \quad(i i)\left\|\nabla F\left(p_{k}\right)\right\|<\frac{1}{k} \quad e \quad(i i i) \Delta F\left(p_{k}\right)>-\frac{1}{k} .
$$

Demonstração dos teoremas.

Antes da demonstração dos Teoremas 3.0.1 e 3.0.2, será feito a demonstração de alguns resultados preliminares, sendo eles uma proposição, um teorema, e um lema, alé do uso da equação, denominada 
de tipo Simons-Calabi, cujo desenvolvimento foi feito na Proposição 2.5.3. Todos os resultados são encontrados em [17]. Na demonstração dos teoremas será mantida a codimensão arbitrária, já que não há grandes mudanças nas contas se restringirmos para o caso de hipersuperfícies. A seguir estão os enunciados, juntamente com a demonstração da proposição e do lema:

Proposição 3.0.5. Seja $M$ uma variedade Riemanniana n-dimensional isométricamente imersa em $L_{p}^{n+p}(c)$. Se $M$ é maximal, a curvatura de Ricci de $M$ satisfaz $\left((n-1) c \delta_{i j}\right) \leq\left(R_{i j}\right)$, e a igualdade vale em toda variedade se, e somente se, $M$ é totalmenge geodésica em $L_{p}^{n+p}(c)$.

Demonstração. Primeiramente, temos que a equação (2.17) pode ser reescrita para codimensão maior que 1 , da forma que

$$
R_{i j}=c(n-1) \delta_{i j}-\sum_{\alpha} h_{\alpha i j} \sum_{k} h_{\alpha k k}+\sum_{\alpha k} h_{\alpha k i} h_{\alpha k j} .
$$

Fixado $p \in M$, podemos escolher um referencial ortonormal $\left\{e_{1}, \ldots, e_{n}\right\}$ de auto-vetores de $h_{\alpha i j}$ onde $\lambda_{\alpha i}$ é auto-valor associado a $e_{i}$, isto é

$$
h_{\alpha i j}=\lambda_{\alpha i} \delta_{i j}
$$

Como $M$ é maximal, então $H=0$. Portanto, 3.1 se reduz a

$$
\begin{aligned}
R_{i j} & =c(n-1) \delta_{i j}+\sum_{\alpha k} h_{\alpha i k} h_{\alpha k j} \\
& =c(n-1) \delta_{i j}+\lambda_{\alpha i} \delta_{i k} \lambda_{\alpha j} \delta_{j k} \\
& =c(n-1) \delta_{i j}+\lambda_{\alpha i} \lambda_{\alpha j} \delta_{i j} .
\end{aligned}
$$

Com isso, como $\lambda_{\alpha i} \lambda_{\alpha j} \delta_{i j} \geq 0$ para todo $i, j$, temos que

$$
R_{i j} \geq c(n-1) \delta_{i j}
$$

Observamos ainda que

$$
\begin{aligned}
R_{i j}=c(n-1) \delta_{i j} & \Leftrightarrow \lambda_{\alpha i} \lambda_{\alpha j} \delta_{i j}=0 \\
& \Leftrightarrow \lambda_{\alpha i}=0, \forall i \\
& \Leftrightarrow \sum_{i} \lambda_{\alpha i}^{2}=0 \\
& \Leftrightarrow \sum_{i} h_{\alpha i i}^{2}=0 \\
& \Leftrightarrow \sum_{\alpha, i, j} h_{\alpha i j}^{2}=0 \\
& \Leftrightarrow S=0 .
\end{aligned}
$$

Portanto, a igualdade vale se, e somente se, $M$ é totalmente geodésica em $L_{p}^{n+p}(c)$.

O próximo teorema pode ser encontrado em [22], onde com as mesmas hipóteses, as mesmas 
desigualdades obtidas no Lema 3.0.4, são obtidas para um único ponto $x \in M$, e não para uma sequencia de pontos $\left\{p_{k}\right\}$ :

Teorema 3.0.6 (Omori-Yau). Seja $M^{n}$ uma variedade Riemanniana completa com curvatura de Ricci limitada por baixo. Seja $f$ uma função $C^{2}$ que é limitada por baixo em M. Então, para todo $\epsilon>0$, existe um ponto $x \in M^{n}$ tal que, em $x$ :

$$
\|\nabla f\|<\epsilon, \quad \Delta f>-\epsilon, \quad f(x)<\inf f+\epsilon .
$$

Por último, o lema que antecede a demonstração dos teoremas 3.0.1 e 3.0.2:

Lema 3.0.7. Seja $M$ uma variedade Riemanniana completa $n$-dimensional, isométricamente imersa em $L_{p}^{n+p}(c)$. Se $M$ for maximal em $L_{p}^{n+p}(c)$, então $S=0$ ou $S \leq-$ cnp.

Demonstração. Pela Proposição 3.0.5, $M$ terá a curvatura de Ricci limitada por baixo. Com isso, estamos nas hipóteses do Teorema 3.0.6. Defina a função $f: M \rightarrow \mathbb{R}$ por

$$
f=\frac{1}{\sqrt{S+a}}
$$

onde $a$ é uma constante positiva qualquer. Então, $f$ é uma função $C^{\infty}$ limitada em $M$. Convencionando que $\Delta f=\nabla f \cdot \nabla f$, e diferenciando (3.3) temos que:

$$
\begin{aligned}
\nabla f & =\nabla\left[(S+a)^{\frac{-1}{2}}\right] \\
& =-\frac{1}{2}(S+a)^{\frac{-3}{2}} \cdot \nabla S \\
& =-\frac{\nabla S}{2(S+a)(\sqrt{S+a})} \\
& =-\frac{f^{3} \cdot \nabla S}{2} .
\end{aligned}
$$

Diferenciando (3.4), e reutilizando sua própria expressão, segue que

$$
\begin{aligned}
\nabla^{2} f & =\frac{\nabla}{2}\left(-f^{3} \cdot \nabla S\right) \\
& =\frac{-\nabla f^{3} \cdot \nabla S}{2}+\frac{-f^{3} \nabla^{2} S}{2} \\
& =-\frac{1}{2}\left(\nabla f^{3} \cdot \nabla S+f^{3} \Delta S\right) \\
& =-\frac{1}{2}\left(3 \nabla f \cdot f^{2} \cdot \nabla S+f^{3} \Delta S\right) \\
& =-\frac{f^{3} \Delta S}{2}+\frac{3 f^{3} \nabla S \cdot f^{2} \cdot \nabla S}{4} \\
& =-\frac{f^{3} \Delta S}{2}+\frac{3 f^{5} \nabla S \cdot \nabla S}{4} \\
& =-\frac{f^{3} \Delta S}{2}+\frac{3 f^{5}\|\nabla S\|^{2}}{4} .
\end{aligned}
$$

Usando (3.4), temos que 


$$
\begin{aligned}
\|\nabla f\|^{2} & =<\nabla f, \nabla f> \\
& =<\frac{f^{3}}{2} \nabla S, \frac{f^{3}}{2} \nabla S> \\
& =\frac{1}{4}<f^{3} \nabla S, f^{3} \nabla S> \\
& =\frac{f^{6}}{4}<\nabla S, \nabla S> \\
& =\frac{f^{6}}{4}\|\nabla S\|^{2} .
\end{aligned}
$$

Agora, usaremos o Teorema 3.0.6 para a função $f$ definida em (3.3), e tomando $\epsilon<1$, teremos que $\|\nabla f\|^{2}<\|\nabla f\|$, logo, existe um ponto $x \in M$ tal que, em $x$ vale:

$$
\frac{f^{6}}{4}\|\nabla S\|^{2}<\epsilon, \quad \Delta f>-\epsilon, \quad f(x)<\inf f+\epsilon .
$$

Note que, multiplicando (3.5) por $f$, e usando as desigualdades de (3.7) temos:

$$
\begin{aligned}
\frac{f^{4}}{2} \Delta S & =-f \Delta f+\frac{3 f^{6}\|\nabla S\|^{2}}{4} \\
\frac{f^{4}}{2} \Delta S & <f \epsilon+\frac{3 f^{6}\|\nabla S\|^{2}}{4} \\
& <f \epsilon+3 \epsilon \\
& <\epsilon(\inf f+\epsilon)+3 \epsilon .
\end{aligned}
$$

Por outro lado, usando o Lema 2.5.2, uma consequência direta de (2.42) é que:

$$
\frac{\Delta S}{2} \geq n c S+\frac{S^{2}}{p}
$$

Portanto por (3.9) e (3.8) temos que

$$
f^{4}\left(n c S+\frac{1}{p} S^{2}\right) \leq \frac{f^{4}}{2} \Delta S<\epsilon(\inf f+\epsilon)+3 \epsilon .
$$

Logo

$$
f^{4}\left(-n c-\frac{1}{p} S\right) S \geq-\epsilon(\inf f+\epsilon)-3 \epsilon
$$

ou seja

$$
\frac{S}{(S+a)^{2}}\left(-n c-\frac{1}{p} S\right) \geq-\epsilon(\inf f+\epsilon)-3 \epsilon .
$$

Com isso, como $\epsilon$ é tomado menor que 1, podemos tomar o limite com $\epsilon \rightarrow 0$, e quando isso ocorre, de (3.7) concluímos que $f$ tende ao ínfimo.

De acordo com a definição de $f$ dada em (3.3), como $\epsilon \rightarrow 0$, e $f$ está tendendo ao ínfimo, então $S(x)$ está tendendo ao seu supremo, quando $\epsilon \rightarrow 0$. Note que $S(x)$ não tende ao infinito quando $\epsilon \rightarrow 0$, pois se fosse verdade, existiria algum $\left(i_{0}, j_{0}\right)$, tal que $h_{i_{0} j_{0}}(x)=+\infty$, e tal fato contraria a hipótese de $M$ ser maximal.

Assim, concluímos que $S$ possui um limitante superior, ou seja, $S$ é função limitada em $M$, pois 
$0 \leq S(x) \leq \sup S \leq+\infty$.

Mas, em contrapartida, quando $\epsilon \rightarrow 0$, de (3.10) podemos concluir que:

$$
\frac{S}{(S+a)^{2}}\left(-n c-\frac{1}{p} S\right) \geq 0 \text {. }
$$

Portanto, $S=0$ resolve a desigualdade, mas se supormos $S \neq 0$, teremos que

$$
S \leq-n c p
$$

Com os resultados anteriores, as demonstrações dos Teoremas 3.0.1 e 3.0.2 são imediatas.

Demonstração. (Teorema 3.0.1) Suponha que $S \neq 0$, então pelo Lema 3.0.7 temos que $S \leq-n c p$ o que é um absurdo pois por hipótese $c \geq 0$. Portanto, $S=0$, isto é, $M$ é totalmente geodésica em $L_{p}^{n+p}(c)$.

Demonstração. (Teorema 3.0.2) Pelo lema 3.0.7, temos que $S \leq-n p c$, mas por hipótese $c<0$, que completa a prova do teorema.

A partir destes resultados, podemos concluir o seguinte fato.

Suponha que $M^{n}$ está imersa em $L_{1}^{n+1}(c), c \geq 0$, onde $M^{n}$ é maximal. Logo, pelo Teorema 3.0 .1 ,

$$
S=0,
$$

ou seja,

$$
\sum_{i j} h_{i j}^{2}=0 .
$$

Consequentemente, $h_{i j}=0, \forall i, j$. Sendo assim, de acordo com a definição de uma imersão ser totalmente umbílica, isto é, se $\forall p \in M^{n}$, a segunda forma fundamental $h=\sum_{i j} h_{i j} \omega_{i} \wedge \omega_{j}$, satisfaz

$$
<h\left(e_{i}, e_{j}\right), \eta>_{p}=\lambda(p)<e_{i}, e_{j}>, \quad \lambda(p) \in \mathbb{R},
$$

onde $\eta$ é um vetor unitário, normal a $M^{n}$ em $p$.

Com isso, definindo $\lambda(p)=0, \forall p \in M^{n}$, temos que a imersão é totalmente umbílica.

Tal fato nos permite ver que, não há motivos para estudar a classificação de hipersuperfícies tipo espaço maximais em $\mathbb{L}_{1}^{n+1}(0)$ e $\mathbb{S}_{1}^{n+1}$, pois as subvariedades já estão classificadas. Como se é conhecido, as subvariedades conexas, completas e totalmente umbílicas de $\mathbb{L}_{1}^{n+1}(0)$ são os espaços afins e as intersecções de espaços afins com hiperesferas.

Já as subvariedades conexas, completas e totalmente umbílicas de $\mathbb{S}_{1}^{n+1}$ são as intersecções de $\mathbb{S}_{1}^{n+1}$ com subespaços afins de $\mathbb{L}_{1}^{n+1}$.

Na demonstração do último teorema desta seção, faremos uso do lema a seguir, cuja demonstração no caso de codimensão $p$ pode ser encontrada em [17]. 
Lema 3.0.8. Seja $M$ uma hipersuperfície tipo espaço, $n$-dimensional, completa e maximal de $\mathbb{H}_{1}^{n+1}$ com $S=n$. Suponha que $h_{i j} \neq h_{l t}$, sempre que $i j \neq l t$, então, $\omega_{i j}=0, \forall i \neq j$.

Demonstração. Primeiramente, note que, como $M$ é hipersuperfície, $c=-1$ e $S$ é constante, de (2.42) temos que

$$
\begin{gathered}
0=\sum\left(h_{i j k}\right)^{2}-n^{2}+n^{2} \\
\therefore h_{i j k}=0 .
\end{gathered}
$$

Escolheremos um referencial ortonormal $\left\{e_{i}\right\}$ tal que $h_{i j}=\lambda_{i} \delta_{i j}$. Feito isso, fixando $i=j$ em (2.6) , teremos que:

$$
\begin{aligned}
& 0=d h_{i i}+\sum_{k} h_{i k} \omega_{k i}+\sum_{k} h_{i k} \omega_{k i} \\
& d h_{i i}=-\sum_{k} h_{i k} \omega_{k i}-\sum_{k} h_{i k} \omega_{k i} \\
& =-h_{i i} \omega_{i i}{ }^{0} h_{i i} \psi_{i i} 0 \\
& =0 \text {. }
\end{aligned}
$$

Logo, isso mostra que $h_{i i}$ é constante. E ainda mais, como $h_{i j}=\lambda_{i} \delta_{i j}$, então $d h_{i j}=0, \forall i, j$, em posse disso, substituindo em (2.6) segue que:

$$
\begin{aligned}
0 & =\sum_{k} h_{i k} \omega_{k j}+\sum_{k} h_{j k} \omega_{k i} \\
& =h_{i i} \omega_{i j}+h_{j j} \omega_{j i} \\
& =\left(h_{i i}-h_{j j}\right) \omega_{i j} .
\end{aligned}
$$

Como $h_{i i} \neq h_{j j}$, então $\omega_{i j}=0, \forall i, j$.

A seguir faremos a demonstração do Teorema 3.0.3 para o caso particular de codimensão 1 . Apenas para facilitar, reescreveremos o teorema para o caso $p=1$.

Teorema 3.0.9. Se $M$ é uma hipersuperfície n-dimensional, tipo espaço, completa, maximal e conexa de $\mathbb{H}_{1}^{n-1}$, satisfazendo $S=n$, então $M$ é isométrica ao produto $\mathbb{H}^{n_{1}}\left(c_{1}\right) \times \mathbb{H}^{n_{2}}\left(c_{2}\right)$, onde $n_{1}$ e $n_{2}$ são inteiros positivos tais que $n_{1}+n_{2}=n$. Mais precisamente, $M$ será isométrica ao cilindro hiperbólico $\mathbb{H}^{1}\left(c_{1}\right) \times \mathbb{H}^{n-1}\left(c_{2}\right)$.

Demonstração. Fixe $p \in M$. Sejam $\left\{e_{i}\right\}$ e $\left\{\omega_{i}\right\}$ o referencial ortonormal e seu co-referencial associado em $p$, repectivamente, definidos em uma vizinhança aberta $U$ de $p$. Agora, vamos definir a distribuição

$$
D_{1}=\left\{v \in T_{p} M: \omega_{1}(v)=\cdots=\omega_{n-1}(v)=0\right\} .
$$


Uma outra forma de definir tal distribuição seria

$$
D_{1}=\left\{v \in T_{p} M: v \in \bigcap_{i=1}^{n-1} \operatorname{Ker}\left(\omega_{i}\right)\right\} .
$$

Primeiramente, verifiquemos que tal distribuição é integrável, isto é, dados $X$ e $Y$ pertencentes a $D_{1}$, mostraremos que $[X, Y]$ também pertence a $D_{1}$. De fato, usando (2.3) e o Lema 3.0.8, temos que

$$
\begin{aligned}
\omega_{i}([X, Y]) & =X\left(\omega_{i}(Y)\right)^{0}-Y\left(\omega_{i}(X)\right)^{0}-d \omega_{i}(X, Y) \\
& =-d \omega_{i}(X, Y) \\
& =-\sum_{j} \omega_{i j} \wedge \omega_{j}(X, Y) \\
& =-\sum_{j} \omega_{i j}(X) \omega_{j}(Y)+\sum_{j} \omega_{i j}(Y) \omega_{j}(X) \\
& =0 .
\end{aligned}
$$

Logo $D_{1}$ é integrável.

Verifiquemos agora que $D_{1}$ é paralela. Para isso, adaptando a nossa distribuição a versão da Definição 1.5.8, os espaços $V_{1}$ e $V_{2}$ são dados por:

$$
\begin{gathered}
V_{1}=\operatorname{span}\left\{e_{n}\right\}, \\
V_{2}=\operatorname{span}\left\{e_{1}, \ldots, e_{n-1}\right\} .
\end{gathered}
$$

Com isso fica fácil de ver que $D_{1}$ é paralela, pois $D_{1}=V_{1}$, e assim, fixando $X_{1} \in V_{1}, v$ uma direção qualquer, usando (2.1) e o Lema 3.0.8 temos:

$$
\begin{aligned}
\nabla_{v} X_{1} & =\nabla_{v} \alpha e_{n} \\
& =\alpha \sum_{j}^{n+1} \underline{\omega}_{n j}(\vartheta) e_{j}^{0} \\
& =0 .
\end{aligned}
$$

Logo $D_{1}$ é distribuição paralela.

Temos então, pelo Teorema de Frobenius, que $D$ é completamente integrável e $M$ pode ser escrita como o produto cartesiano de duas hipersuperfícies tais que $M=M_{1} \times M_{2}$.

Ainda mais, de acordo com a demonstraçã do paralelismo de $D_{1}$, dado que $D_{1}=\operatorname{span}\left\{e_{n}\right\}$, logo, $\operatorname{dim}\left(D_{1}\right)=1$, sendo assim, se $M_{1}$ é a variedade integral que passa por $p$, então ela tem dimensão 1 , então temos que $M_{1}$ é difeomorfo a $\mathbb{R}$. Aqui $\mathbb{H}^{n}(r)$ denota o espaço hiperbólico de curvatura constante $r<0$, e pode ser considerado como a componente de $\mathbb{H}_{0}^{n}(r)$ por $(0, \ldots, 0, r)$.

Assim, podemos considerar $\mathbb{R}$ como $\mathbb{H}^{1}\left(c_{1}\right)$.

Definindo agora $D_{2}=V_{2}$, se $M_{2}$ é a variedade integral maximal que passa por $p$, então a dimensão de $M_{2}$ é igual a $n-1$, que também é a dimensão de $V_{2}$. De maneira análoga, podemos 
usar a identificação contida na demonstração de 3.0 .3 em [17], e consideramos $M_{2}=\mathbb{H}^{n-1}\left(c_{2}\right)$. Com isso, $M=M_{1} \times M_{2}$, ou seja, $M$ é isométrica ao produto $\mathbb{H}^{1}\left(c_{1}\right) \times \mathbb{H}^{n-1}\left(c_{2}\right)$. 


\section{Capítulo 4}

\section{Uma caracterização de cilindros hiperbólicos}

Neste capítulo estudaremos as hipersuperfícies maximais, completas no espaço anti-de Sitter $\mathbb{H}_{1}^{n+1}$, com $n-1$ curvaturas com mesmo sinal em todo lugar, com o objetivo de caracterizar os cilindros hiperbólicos $\mathbb{H}^{1}\left(c_{1}\right) \times \mathbb{H}^{n-1}\left(c_{2}\right)$. Os dois resultados principais estudados neste capítulo são:

Teorema 4.0.1. Seja $M^{n}$ uma hypersuperfície completa maximal em $\mathbb{H}_{1}^{n+1}(-1)$. Se $M^{n}$ possui curvatura escalar constante, $n-1$ curvaturas principais com o mesmo sinal em toda variedade, e curvatura de Gauss - Kronecker limitada longe do zero, então $M^{n}$ é isométrica ao cilindro hiperbólico $\mathbb{H}^{1}\left(c_{1}\right) \times \mathbb{H}^{n-1}\left(c_{2}\right)$.

Teorema 4.0.2. Seja $M^{n}$ uma hipersuperficie completa maximal em $\mathbb{H}_{1}^{n+1}(-1)$ com curvatura de Gauss-Kronecker constante não-nula. Se $M^{n}$ tem $(n-1)$ curvaturas principais com o mesmo sinal em toda hipersuperfície, então $M^{n}$ é isométrica ao cilindro hiperbólico $\mathbb{H}^{1}\left(c_{1}\right) \times \mathbb{H}^{n-1}\left(c_{2}\right)$.

A seguir faremos a demonstração de alguns lemas que serão fundamentais na demonstração do teorema 4.0 .1

Lema 4.0.3. Seja $M^{n}$ uma hipersuperfície maximal, com curvatura de Gauss-Kronecker limitada longe do zero. Defina $F=\log \left|\operatorname{det}\left(h_{i j}\right)\right|$, onde $h_{i j}$ são componentes da matriz da segunda forma fundamental, no referencial ortonormal $\left\{e_{i}\right\}$. Então:

$$
\Delta F=-\sum_{i, j, k} \frac{1}{\lambda_{i} \lambda_{j}} h_{i j k}^{2}+\sum_{i, k} \frac{1}{\lambda_{i}} h_{i i k k} .
$$

Demonstração. Para cada ponto $p \in M$, podemos escolher um referencial ortonormal $\left\{e_{1}, \ldots, e_{n}\right\}$ de auto-vetores de $h_{i j}$ e seja $\lambda_{i}$ o auto-valor associado a $e_{i}$, isto é

$$
h_{i j}=\lambda_{i} \delta_{i j}
$$

De acordo com [23], $F_{k}=\sum_{i j} h^{i j} h_{i j k}$, onde $h^{i j}$ é o elemento inverso de cada $h_{i j}$, ou seja, $h^{i j}=\frac{1}{\lambda_{i}} \delta_{i j}$. Como

$$
\delta_{i j}=\sum_{l} h_{i l} h^{l j},
$$


podemos escrever

$$
\begin{aligned}
h^{i j} & =\sum_{m} h^{i m} \delta_{m j} \\
& =\sum_{m l} h^{i m} h^{l j} h_{m l} .
\end{aligned}
$$

Então, $F_{k}=\sum_{i j}\left(\sum_{m l} h^{i m} h^{l j} h_{m l}\right) h_{i j k} \mathrm{e}$

$$
\begin{aligned}
F_{k k} & =\sum_{i j}\left(\sum_{m l} h^{i m} h^{l j} h_{m l}\right)_{k} h_{i j k}+\sum_{i j}\left(\sum_{m l} h^{i m} h^{l j} h_{m l}\right) h_{i j k k} \\
& =\sum_{i j}(\underbrace{\sum_{m l} h^{i m} h^{l j} h_{m l}}_{(*)})_{k} h_{i j k}+\sum_{i j} h^{i j} h_{i j k k} .
\end{aligned}
$$

Analisando o termo $(*)$ temos que:

$$
\begin{aligned}
\left(\sum_{m l} h^{i m} h^{l j} h_{m l}\right)_{k} & =\sum_{m l}\left(h_{k}^{i m} h^{l j} h_{m l}+h^{i m} h_{k}^{l j} h_{m l}+h^{i m} h^{l j} h_{m l k}\right) \\
& =\sum_{m l} h^{l j}\left(h_{k}^{i m} h_{m l}+h^{i m} h_{m l k}\right)+\sum_{m l} h^{i m} h_{k}^{l j} h_{m l} .
\end{aligned}
$$

Derivando (4.2) em relação a $k$ segue que:

$$
0=\left(\sum_{l} h_{i l} h^{l j}\right)_{k}=\sum_{l} h_{i l k} h^{l j}+\sum_{l} h_{i l} h_{k}^{l j},
$$

isto é:

$$
\begin{aligned}
& \sum_{l} h_{i l k} h^{l j}=-\sum_{l} h_{i l} h_{k}^{l j} \\
& -\sum_{l} h_{i l k} h^{l j}=\sum_{l} h_{i l} h_{k}^{l j} .
\end{aligned}
$$

Usando tais identidades, arrumando os índices, teremos:

$$
\begin{gathered}
\sum_{m} h^{m i} h_{l m k}=-\sum_{m} h_{l m} h_{k}^{m i}, \\
\sum_{l} h_{m l} h_{k}^{l j}=-\sum_{l} h_{m l k} h^{l j} .
\end{gathered}
$$

Substituindo (4.4) e (4.5) em (4.3), fazendo as trocas necessárias dos índices, obtemos que: 


$$
\begin{aligned}
\sum_{m l} h^{l j}(h_{k}^{i m} h_{m l}+\underbrace{h^{i m} h_{m l k}}_{(4.4)}) & +\sum_{m l} h^{i m} h_{k}^{l j} h_{m l}= \\
= & \sum_{m l} h^{l j}\left(h_{k}^{i m} h_{m l}-h_{l m} h_{k}^{m i}\right)+ \\
& \sum_{m l} h^{i m} \underbrace{h_{k}^{l j} h_{m l}}_{(4.5)} \\
= & -\sum_{m l} h^{i m} h^{l j} h_{m l k} .
\end{aligned}
$$

Utilizando as relações obtidas concluímos o desejado, isto é

$$
\begin{aligned}
\Delta F & =\sum_{k} F_{k k} \\
& =-\sum_{i, j, k} \frac{1}{\lambda_{i} \lambda_{j}} h_{i j k}^{2}+\sum_{i, k} \frac{1}{\lambda_{i}} h_{i i k k} .
\end{aligned}
$$

Para uso futuro, temos que a primeira parcela da igualdade anterior pode ser escrita como

$-\sum_{i j k} \frac{1}{\lambda_{i} \lambda_{j}} h_{i j k}^{2}=-\sum_{i} \frac{1}{\lambda_{i}^{2}} h_{i i i}^{2}-\sum_{i=j \neq k} \frac{1}{\lambda_{i}^{2}} h_{i i k}^{2}-\sum_{i=k \neq j} \frac{1}{\lambda_{i} \lambda j} h_{i i j}^{2}-\sum_{i \neq j=k} \frac{1}{\lambda_{i} \lambda_{j}} h_{j j i}^{2}-\sum_{i \neq j \neq k} \frac{1}{\lambda_{i} \lambda_{j}} h_{i j k}^{2}$

onde os índices $, i, j, k$ foram separados de forma conveniente.

Lema 4.0.4. Se $M^{n}$ é uma hipersuperfície tipo espaço maximal em $\mathbb{H}_{1}^{n+1}(-1)$, então a expressão de (2.15) se reduz a

$$
\Delta h_{i j}=(S-n) h_{i j}
$$

Demonstração. Primeiramente, vale observar que $h_{k i k}=h_{k k i}$ por (2.9). Se derivarmos tal igualdade em relação a $j$ e fizermos o somatório em $k$, teremos que

$$
\sum_{k} h_{k i k j}=\sum_{k} h_{k k i j}
$$

e, como por hipótese $M^{n}$ é maximal, ou seja

$$
H=\sum_{k} h_{k k}=0,
$$

concluímos então que

$$
\sum_{k} h_{k i k j}=\sum_{k} h_{k k i j}=0 .
$$

Por (2.15) segue que 


$$
\begin{aligned}
\Delta h_{i j}= & \sum_{m k} h_{k m} R_{m i j k}+\sum_{m k} h_{i m} R_{m k j k} \\
= & \sum_{m k} h_{m i}\left(K_{m k j k}+h_{m k} h_{k j}-h_{m j} h_{k k}\right)+\sum_{m k} h_{k m}\left(K_{m i j k}+h_{m k} h_{i j}-h_{m j} h_{i k}\right) \\
= & \sum_{m k} h_{m i} K_{m k j k}+\sum_{m k} h_{m i} h_{m k} h_{k j}-\sum_{m k} h_{m i} h_{m j} h_{k k}+\sum_{m k} h_{k m} K_{m i j k}+ \\
& \sum_{m k} h_{k m} h_{m k} h_{i j}-\sum_{m k} h_{k m} h_{m j} h_{i k} \\
= & \sum_{m k} h_{m i} K_{m k j k}+\sum_{m k} h_{m i} h_{m k} h_{k j}+\sum_{m k} h_{k m} K_{m i j k}+\sum_{m k} h_{k m}^{2} h_{i j}-\sum_{m k} h_{k m} h_{m j} h_{i k} \\
= & \sum_{m k} h_{m i}\left(\delta_{m k} \delta_{k j}-\delta_{m j} \delta_{k k}\right)+\sum_{m k} h_{k m}\left(\delta_{m k} \delta_{i j}-\delta_{m j} \delta_{i k}\right)+S h_{i j} \\
= & -\sum_{m k} h_{m i} \delta_{m j}+\sum_{m k} h_{m i} \delta_{m k} \delta_{k j}-\sum_{m k} h_{k m} \delta_{m j} \delta_{i k}+\sum_{m k} h_{k m} \delta_{m k} \delta_{i j}+S h_{i j} \\
= & -\sum_{m k} h_{m i} \delta_{m j}+\sum_{m k} h_{m i} \delta_{m k} \delta_{k j}-\sum_{m k} h_{k m} \delta_{m j} \delta_{i k}+\sum_{k} h_{k k} \delta_{i j}+S h_{i j} \\
= & -\sum_{m k} h_{m i} \delta_{m j}+\sum_{m k} h_{m i} \delta_{m k} \delta_{k j}-\sum_{m k} h_{k m} \delta_{m j} \delta_{i k}+S h_{i j} \\
= & -\sum_{m k} h_{m i} \delta_{m j}+\sum_{m} h_{m i} \delta_{m j}-\sum_{m} h_{i m} \delta_{m j}+S h_{i j} \\
= & -\sum_{m k} h_{m i} \delta_{m j}+S h_{i j} \\
= & -\sum_{k} h_{i j}+S h_{i j} \\
= & -n h_{i j}+S h_{i j} \\
= & S h_{i j} . \\
&
\end{aligned}
$$

A seguir serão feitas algumas manipulações algébricas com o objetivo de encontrar uma expressão tal que possamos retirar mais informações sobre os limitantes de $S \Delta F$.

Tomando $j=i$ na igualdade anterior, segue que

$$
\Delta h_{i i}=(S-n) h_{i i}=(S-n) \lambda_{i}
$$

donde segue por (2.14) que

$$
\begin{aligned}
\sum_{i k} \frac{1}{\lambda_{i}} h_{i i k k} & =\sum_{i} \frac{1}{\lambda_{i}}\left(\sum_{k} h_{i i k k}\right) \\
& =\sum_{i}(S-n) \\
& =n(S-n) .
\end{aligned}
$$


Observamos que:

$$
\begin{aligned}
\Delta h_{i j}^{2} & =h_{i j} \Delta h_{i j}+h_{i j} \Delta h_{i j}+2 \nabla h_{i j} \nabla h_{i j} \\
& =2 h_{i j} \Delta h_{i j}+2 \sum_{k} h_{i j k}^{2} \\
& =2 h_{i j}(S-n) h_{i j}+2 \sum_{k} h_{i j k}^{2} \\
& =2\left(h_{i j}^{2}(S-n)+\sum_{k} h_{i j k}^{2}\right) .
\end{aligned}
$$

Com isso, fazendo a soma em $i, j$ na última igualdade, obtemos

$$
\begin{aligned}
\Delta S & =2\left(\sum_{i j} h_{i j}^{2}(S-n)+\sum_{i j k} h_{i j k}^{2}\right. \\
& =2\left(S(S-n)+\sum_{i j k} h_{i j k}^{2},\right.
\end{aligned}
$$

ou seja,

$$
\frac{1}{2} \Delta S=S(S-n)+\sum_{i j k} h_{i j k}^{2}
$$

Separando os indices $i, j, k$ de forma conveniente, podemos reescrever a igualdade anterior como:

$$
0=\frac{1}{2} \Delta S=S(S-n)+\sum_{i} h_{i i i}^{2}+3 \sum_{i \neq j} h_{i i j}^{2}+\sum_{i \neq j \neq k} h_{i j k}^{2} .
$$

De $(4.1),(4.7),(4.10)$ e (4.6), obtemos a seguinte relação:

$$
\begin{aligned}
S \Delta F= & S\left(-\sum_{i j k} \frac{1}{\lambda_{i} \lambda_{j}} h_{i j k}^{2}+\sum_{i k} \frac{1}{\lambda_{i}} h_{i i k k}\right) \\
= & S\left(-\sum_{i j k} \frac{1}{\lambda_{i} \lambda_{j}} h_{i j k}^{2}\right)+S n(S-n) \\
= & -\sum_{i} \frac{S}{\lambda_{i}^{2}} h_{i i i}^{2}-\sum_{i=j \neq k} \frac{S}{\lambda_{i}^{2}} h_{i i k}^{2}-\sum_{i=k \neq j} \frac{S}{\lambda_{i} \lambda j} h_{i i j}^{2}-\sum_{i \neq j=k} \frac{S}{\lambda_{i} \lambda_{j}} h_{j j i}^{2}+ \\
& -\sum_{i \neq j \neq k} \frac{S}{\lambda_{i} \lambda_{j}} h_{i j k}^{2}+S n(S-n) \\
= & -\sum_{i} \frac{S}{\lambda_{i}^{2}} h_{i i i}^{2}-\sum_{i=j \neq k} \frac{S}{\lambda_{i}^{2}} h_{i i k}^{2}-\sum_{i=k \neq j} \frac{S}{\lambda_{i} \lambda j} h_{i i j}^{2}-\sum_{i \neq j=k} \frac{S}{\lambda_{i} \lambda_{j}} h_{j j i}^{2}+ \\
& -\sum_{i \neq j \neq k} \frac{S}{\lambda_{i} \lambda_{j}} h_{i j k}^{2}+n\left[\frac{S(S-n)}{3}+\frac{2 S(S-n)}{3}\right] .
\end{aligned}
$$

Agora note que, se S é constante, então $\Delta S=0$, ou ainda mais, $\frac{1}{2} \Delta S=0$ e com isso de (4.10) 
temos:

$$
\frac{n S(S-n)}{3}=-\frac{n}{3} \sum_{i} h_{i i i}^{2}-n \sum_{i \neq j} h_{i i j}^{2}-\frac{n}{3} \sum_{i \neq j \neq k} h_{i j k}^{2} .
$$

Entao, no caso particular de $S$ constante, (4.11) pode ser reescrita como:

$$
\begin{aligned}
S \Delta F= & -\sum_{i} \frac{S}{\lambda_{i}^{2}} h_{i i i}^{2}-\sum_{i=j \neq k} \frac{S}{\lambda_{i}^{2}} h_{i i k}^{2}-\sum_{i=k \neq j} \frac{S}{\lambda_{i} \lambda j} h_{i i j}^{2}-\sum_{i \neq j=k} \frac{S}{\lambda_{i} \lambda_{j}} h_{j j i}^{2}+ \\
& -\sum_{i \neq j \neq k} \frac{S}{\lambda_{i} \lambda_{j}} h_{i j k}^{2}-\frac{n}{3} \sum_{i} h_{i i i}^{2}-n \sum_{i \neq j} h_{i i j}^{2}-\frac{n}{3} \sum_{i \neq j \neq k} h_{i j k}^{2}+\frac{2 n S(S-n)}{3} \\
= & -\sum_{i}\left(\frac{S}{\lambda_{i}^{2}}+\frac{n}{3}\right) h_{i i i}^{2}-\sum_{i \neq j}\left(\frac{S}{\lambda_{i}^{2}}+\frac{2 S}{\lambda_{i} \lambda_{j}}+n\right) h_{i i j}^{2}-\sum_{i \neq j \neq k}\left(\frac{S}{\lambda_{i} \lambda_{j}}+\frac{n}{3}\right) h_{i j k}^{2}+ \\
& +\frac{2 n S(S-n)}{3} .
\end{aligned}
$$

Reorganizando os índices, e utilizando (2.9) temos:

$$
\begin{aligned}
3 \sum_{i \neq j \neq k} \frac{1}{\lambda_{i} \lambda_{j}} h_{i j k}^{2} & =\sum_{i \neq j \neq k}\left(\frac{1}{\lambda_{i} \lambda_{j}}+\frac{1}{\lambda_{i} \lambda_{k}}+\frac{1}{\lambda_{j} \lambda_{k}}\right) h_{i j k}^{2} \\
& =\sum_{i \neq j \neq k}\left(\frac{\lambda_{i}+\lambda_{j}+\lambda_{k}}{\lambda_{i} \lambda_{j} \lambda_{k}}\right) h_{i j k}^{2} .
\end{aligned}
$$

Substituindo (4.13) em (4.12), e separando os índices $n$, temos:

$$
\begin{aligned}
S \Delta F= & -\sum_{i}^{n}\left(\frac{S}{\lambda_{i}^{2}}+\frac{n}{3}\right) h_{i i i}^{2}-\sum_{i \neq j}^{n-1}\left(\frac{S}{\lambda_{i}^{2}}+\frac{2 S}{\lambda_{i} \lambda_{j}}+n\right) h_{i i j}^{2}-\sum_{i=1}^{n-1}\left(\frac{S}{\lambda_{i}^{2}}+\frac{2 S}{\lambda_{i} \lambda_{n}}+n\right) h_{i i n}^{2} \\
& -\sum_{j=1}^{n-1}\left(\frac{S}{\lambda_{n}^{2}}+\frac{2 S}{\lambda_{j} \lambda_{n}}+n\right) h_{n n j}^{2}-\frac{1}{3} \sum_{i \neq j \neq k}^{n}\left[\left(\frac{\lambda_{i}+\lambda_{j}+\lambda_{k}}{\lambda_{i} \lambda_{j} \lambda_{k}}\right) S+n\right] h_{i j k}^{2} \\
& +\frac{2 n S(S-n)}{3} .
\end{aligned}
$$

No que segue, mostraremos que no caso onde $S$ é constante, $S \Delta F \leq \frac{2 n S(S-n)}{3}$. Para provar o desejado, basta verificar que os 5 primeiros termos de (4.14) são não positivos. Os dois primeiros são claramente não positivos, restando a análise dos 3 termos seguintes.

- Análise do termo $\frac{\lambda_{i}+\lambda_{j}+\lambda_{k}}{\lambda_{i} \lambda_{j} \lambda_{k}}$.

Se $i, j, k \neq n$, o termo é claramente positivo. Suponha que o índice $i=n$. Assim, lembrando que $M^{n}$ é maximal, podemos reescrever o termo da seguinte forma:

$$
\frac{\lambda_{n}+\lambda_{j}+\lambda_{k}}{\lambda_{n} \lambda_{j} \lambda_{k}}=\frac{-\sum_{l \neq n} \lambda_{l}+\lambda_{j}+\lambda_{k}}{\lambda_{n} \lambda_{j} \lambda_{k}} .
$$

Sendo assim, é fácil ver que o denominador é negativo, mas da forma que foi reescrito, seu 
numerador também será negativo, com isso, todos os casos estão cobertos, e $\forall i, j, k$, temos que $\frac{\lambda_{i}+\lambda_{j}+\lambda_{k}}{\lambda_{i} \lambda_{j} \lambda_{k}}>0$.

- Análise do termo $\left(\frac{S}{\lambda_{i}^{2}}+\frac{2 S}{\lambda_{i} \lambda_{n}}+n\right)$.

Observe que:

$$
-\lambda_{n}=\sum_{i}^{n-1} \lambda_{i} \Rightarrow \lambda_{n}^{2}=\left(\sum_{i}^{n-1} \lambda_{i}\right)^{2},
$$

e

$$
\left(\sum_{i}^{n-1} \lambda_{i}\right)^{2}>\sum_{i}^{n-1} \lambda_{i}^{2}
$$

Com essas duas informações concluímos que

$$
\lambda_{n}^{2}>\sum_{i}^{n-1} \lambda_{i}^{2}
$$

e portanto

$$
\begin{gathered}
n \lambda_{n}^{2}>\sum_{i}^{n-1} \lambda_{i}^{2}=S . \\
\Rightarrow n \lambda_{n}^{2}>S, \\
\Rightarrow n>\frac{S}{\lambda_{n}^{2}} .
\end{gathered}
$$

Com isso, obtemos a seguinte desigualdade

$$
\begin{aligned}
\sum_{i=1}^{n-1}\left(\frac{S}{\lambda_{i}^{2}}+\frac{2 S}{\lambda_{i} \lambda_{n}}+n\right) h_{\text {iin }}^{2} & \geq \sum_{i=1}^{n-1}\left(\frac{S}{\lambda_{i}^{2}}+\frac{2 S}{\lambda_{i} \lambda_{n}}+\frac{S}{\lambda_{n}^{2}}\right) h_{\text {iin }}^{2} \\
& =\sum_{i=1}^{n-1} S\left(\frac{1}{\lambda_{i}}+\frac{1}{\lambda_{n}}\right)^{2} h_{\text {iin }}^{2} \\
& \geq 0 .
\end{aligned}
$$

Logo o termo analisado também será não-positivo.

Agora nos resta analisar o sinal do somando

$$
\gamma=\sum_{j=1}^{n-1}\left(\frac{S}{\lambda_{n}^{2}}+\frac{2 S}{\lambda_{j} \lambda_{n}}+n\right) h_{n n j}^{2}
$$

Diferentemente da análise dos outros termos, será necessário considerar termos em $\sum_{i}^{n}\left(\frac{S}{\lambda_{i}^{2}}+\right.$ $\left.\frac{n}{3}\right) h_{i i i}^{2}$ e $\sum_{i \neq j}^{n-1}\left(\frac{S}{\lambda_{i}^{2}}+\frac{2 S}{\lambda_{i} \lambda_{j}}+n\right) h_{i i j}^{2}$ que compensem de alguma forma os termos não-positivos em $\gamma$. Para isso, vamos fazer uso do lema 2.5.1. E importante observar que com esta estratégia o sinal dos termos restantes não será alterado. 
Diferenciando $\sum_{i} h_{i i}=0$ e $S=\sum_{i k} h_{i k}^{2}$, temos:

$$
\sum_{j} h_{i i k}=0
$$

$\mathrm{e}$

$$
\sum_{i k} 2 h_{i k} h_{i k j}=0 \Rightarrow \sum_{i} \lambda_{i} h_{i i j}=0, j=1, \ldots, n .
$$

Definindo $\beta_{i j}=h_{i i j}$, temos que $\lambda_{i}$ e $\beta_{i j}$ são números reais e devido ao fato de $M^{n}$ ser maximal, satisfazem as condições do Lema 2.5.1. Portanto, seja $g_{j} \in\{1, \ldots, n\}$ o maior índice tal que $\left|h_{g_{j} g_{j} j}\right|=\max \left\{\left|h_{i i j}\right|, i=1, \ldots n\right\}$. De acordo com o lema, temos apenas os casos $\max \left\{\left|h_{i i j}\right|, i=\right.$ $1, \ldots n\} \neq\left|h_{n n j}\right|$ ou $\left|h_{n n j}\right|=0$.

Seja agora $g_{j} \in I_{n-1}=\{1, \ldots, n-1\}$, o índice tal que $\left|h_{g_{j} g_{j} j}\right|=\max \left\{\left|h_{i i j}\right|, i=1, \ldots, n\right\}$.

Consideraremos os seguintes subconjuntos de $I_{n-1}$ :

$$
\begin{gathered}
A=\left\{j \in I_{n-1}: g_{j}=j\right\}, \\
B=\left\{j \in I_{n-1}: g_{j} \notin\{j, n\}\right\}, \\
C=\left\{j \in I_{n-1}: g_{j}=n\right\} .
\end{gathered}
$$

Reenumerando $e_{1}, \ldots, e_{n}$, se necessário, podemos assumir que os conjuntos estão dispostos da seguinte forma:

$$
\begin{gathered}
A=\emptyset \text { ou } A=\{1, \ldots, r\}, \\
B=\emptyset \text { ou } B=\{r+1, \ldots, t\}, \\
C=\emptyset \text { ou } C=\{t+1, \ldots, n-1\} .
\end{gathered}
$$

E para nosso estudo adotaremos a seguinte convenção:

Se $A=\emptyset$ então $r=0$,

Se $B=\emptyset$ então $t=r$,

Se $C=\emptyset$ então $t=n-1$.

Usando tais conjuntos, reescreveremos (4.14) de maneira mais conveniente, para que possamos analisar os sinais dos somandos:

$$
\begin{aligned}
S \Delta F= & -\sum_{i=r+1}^{n}\left(\frac{S}{\lambda_{i}^{2}}+\frac{n}{3}\right) h_{i i i}^{2}-\sum_{i \neq j \in I_{n-1},(i, j) \neq\left(g_{j}, j\right)}\left(\frac{S}{\lambda_{i}^{2}}+\frac{2 S}{\lambda_{i} \lambda_{j}}+n\right) h_{i i j}^{2} \\
& -\sum_{i=1}^{n-1}\left(\frac{S}{\lambda_{i}^{2}}+\frac{2 S}{\lambda_{i} \lambda_{n}}+n\right) h_{i i n}^{2}-\sum_{j=1}^{r}\left[\left(\frac{S}{\lambda_{n}^{2}}+\frac{2 S}{\lambda_{j} \lambda_{n}}+n\right) h_{n n j}^{2}+\left(\frac{S}{\lambda_{j}^{2}}+\frac{n}{3}\right) h_{j j j}^{2}\right] \\
& -\sum_{j=r+1}^{t}\left[\left(\frac{S}{\lambda_{n}^{2}}+\frac{2 S}{\lambda_{j} \lambda_{n}}+n\right) h_{n n j}^{2}+\left(\frac{S}{\lambda_{g_{j}}^{2}}+\frac{2 S}{\lambda_{j} \lambda_{g_{j}}}+n\right) h_{g_{j} g_{j} j}^{2}\right] \\
& -\frac{1}{3} \sum_{i \neq j \neq k}^{n}\left[\left(\frac{\lambda_{i}+\lambda_{j}+\lambda_{k}}{\lambda_{i} \lambda_{j} \lambda_{k}}\right) S+n\right] h_{i j k}^{2}+\frac{2 n S(S-n)}{3} .
\end{aligned}
$$


Como $h_{j j j}^{2} \geq h_{n n j}^{2}, \quad j=1, \ldots, r$, segue que

$$
\begin{aligned}
\sum_{j=1}^{r}\left(\frac{S}{\lambda_{n}^{2}}+\frac{2 S}{\lambda_{j} \lambda_{n}}+n\right) h_{n n j}^{2}+\sum_{j=1}^{r}\left(\frac{S}{\lambda_{j}^{2}}+\frac{n}{3}\right) h_{j j j}^{2} & \geq \sum_{j=1}^{r}\left(\frac{S}{\lambda_{n}^{2}}+\frac{2 S}{\lambda_{j} \lambda_{n}}+\frac{S}{\lambda_{j}^{2}}+\frac{4 n}{3}\right) h_{n n j}^{2} \\
& =\sum_{j=1}^{r}\left[S\left(\frac{1}{\lambda_{n}}+\frac{1}{\lambda_{j}}\right)^{2}+\frac{4 n}{3}\right] h_{n n j}^{2} \\
& \geq 0 .
\end{aligned}
$$

Como $h_{g_{j} g_{j} j}^{2}>h_{n n j}^{2}, \quad j=r+1, \ldots, t$, temos

$$
\begin{aligned}
& \sum_{j=r+1}^{t}\left(\frac{S}{\lambda_{n}^{2}}+\frac{2 S}{\lambda_{j} \lambda_{n}}+n\right) h_{n n j}^{2}+\sum_{j=r+1}^{t}\left(\frac{S}{\lambda_{g_{j}}^{2}}+\frac{2 S}{\lambda_{j} \lambda_{g_{j}}}+n\right) h_{g_{j} g_{j} j}^{2} \\
& \geq \sum_{j=r+1}^{t}\left[\frac{S}{\lambda_{n}^{2}}+\frac{S}{\lambda_{g_{j}}^{2}}+2 n+\frac{2 S}{\lambda_{j}}\left(\frac{1}{\lambda_{g_{j}}}+\frac{1}{\lambda_{n}}\right)\right] h_{n n j}^{2} . \\
& \geq 0
\end{aligned}
$$

Portanto, segue da análise feita anteriormente que no caso particular de $S$ constante

$$
S \Delta F \leq \frac{2 n S(S-n)}{3} .
$$

Com todas as ferramentas em mãos, podemos fazer a demonstração do Teorema 4.0.1.

Demonstração. (Teorema 4.0.1) Pela proposição 3.0.5, cada componente $i, j$ da curvatura de Ricci é maior ou igual que $-(n-1)$, seja $T_{1} M$ o fibrado tangente unitário de $M^{n}$, dado $v \in T_{1} M$ temos:

$$
\operatorname{Ric}(v, v) \geq-(n-1), \quad \forall v \in T_{1} M .
$$

De acordo com (4.18), a curvatura de Ricci de $M^{n}$ é limitada por baixo. Como por hipótese, a curvatura de Gauss-Kronecker é limitada longe do zero, então a função $F=\log \left|\operatorname{det}\left(h_{i j}\right)\right|$ é uma função $C^{2}$ que também é limitada por baixo.(Note que a curvatura de Gauss-Kronecker pode ser definida como $\left.\operatorname{det}\left(h_{i j}\right)\right)$.

Logo podemos aplicar o Lema 3.0.4 a $F$ e obtermos uma sequência de pontos $\left\{p_{k}\right\}$ em $M^{n}$ tal que:

$$
\lim _{k \rightarrow+\infty} F\left(p_{k}\right)=\inf F, \quad \lim _{k \rightarrow+\infty}\left|\nabla F\left(p_{k}\right)\right|=0, \quad \liminf _{k \rightarrow+\infty} \Delta F\left(p_{k}\right) \geq 0 .
$$

Calculando (4.17) nos pontos $\left\{p_{k}\right\}$ obtidos pelo Lema 3.0.4, e tomando o lim inf, teremos:

$$
\liminf _{k \rightarrow+\infty} S \Delta F\left(p_{k}\right)=S \liminf _{k \rightarrow+\infty} \Delta F\left(p_{k}\right) \geq 0
$$

Sendo assim, temos que

$$
0 \leq S \Delta F \leq \frac{2 n S(S-n)}{3}
$$

isto é, 


$$
0 \leq \Delta F \leq \frac{2 n(S-n)}{3}
$$

e portanto:

$$
0 \leq \frac{2 n(S-n)}{3} \Rightarrow S \geq n .
$$

Mas, as hipóteses do Teorema 3.0.2 são satisfeitas pelas hipóteses do Teorema 4.0.1, logo, temos também que $S \leq n$. Portanto, $S=n$.

Logo pelo Teorema 3.0.9 temos que $M^{n}$ é isométrico ao cilindro hiperbólico $\mathbb{H}^{1}\left(c_{1}\right) \times \mathbb{H}^{n-1}\left(c_{2}\right)$.

Segue do Teorema 4.0.1 o seguinte resultado:

Corolario 4.0.5. Seja $M^{4}$ uma hipersuperfície maximal em $\mathbb{H}_{1}^{5}(-1)$ com curvatura escalar constante. Se $M^{4}$ tem curvatura de Gauss-Kronecker negativa e limitada longe do zero em toda hipersuperfície, então $M^{4}$ é isométrica ao cilindro hiperbólico $\mathbb{H}^{1}\left(c_{1}\right) \times \mathbb{H}^{3}\left(c_{2}\right)$.

Demonstração. Note que, como $K<0$, e $K$ é o produto das curvaturas principais de $M^{4}$, então, obrigatoriamente 3 curvaturas principais possuem o mesmo sinal. Portanto, $M^{4}$ tem $4-1$ curvaturas principais com o mesmo sinal.

Com isso, todas as hipóteses do Teorema 4.0.1 estão satisfeitas e o resultado segue direto.

Para a demonstração do Teorema 4.0.2, faremos uso de dois lemas aqui enunciados e provados.

Primeiramente veja que, $M^{n}$ é completa, de (4.18) temos que a curvatura de Ricci é limitada por baixo, e $S \geq 0$ é função diferenciável, como foi visto na demonstração do teorema anterior.

Então, como todas as hipóteses do Lema 3.0.4 são satisfeitas, podemos aplicá-lo para a função $\mathrm{S}$, a fim de obtermos uma sequência de pontos $\left\{p_{k}\right\}$ tal que:

$$
\text { i) } \left.\left.S\left(p_{k}\right)<\inf S+\frac{1}{k}, \quad i i\right)\left|\nabla S\left(p_{k}\right)\right|<\frac{1}{k}, \quad i i i\right) \Delta S\left(p_{k}\right)>-\frac{1}{k} \text {. }
$$

Temos por 3.0.2 que $S \leq n$, e note que, como $\lambda_{n}=-\sum_{i}^{n-1} \lambda_{i}$, então qualquer sequência envolvendo algum $\lambda_{j}$, será limitada pela soma dos demais, sendo assim, sabemos que toda sequência limitada possui subsequência convergente, então é possível tomarmos, se necessário, subsequência de pontos $\left\{p_{k}\right\}$ tal que:

$$
\lim _{k \rightarrow \infty} \lambda_{i}\left(p_{k}\right)=\tilde{\lambda_{i}}, \quad \tilde{\lambda_{i}} \neq 0 .
$$

O lema a seguir é uma extensão do Lema 2.5.1 para o caso em que a curvatura escalar $R$ não é constante, o que é útil pois o Teorema 4.0.2 não possui nenhum hipótese sobre $R$. 
Lema 4.0.6. Seja $M^{n}$ uma hipersuperfície maximal, completa, com curvatura de Gauss-Kronecker constante não-nula em $\mathbb{H}_{1}^{n+1}$. Se existe uma subsequência de pontos $\left\{p_{k}\right\}$ definida como em (4.19) e (4.20) tal que:

$$
\left|h_{n n j}\left(p_{k}\right)\right|=\max \left\{\left|h_{i i j}\left(p_{k}\right)\right|, i=1, \ldots, n\right\},
$$

então $\lim _{k \rightarrow \infty} h_{i i j}\left(p_{k}\right)=0, \quad i=1, \ldots, n$.

Demonstração. Para todo ponto $p$ fixo em $M^{n}$, podemos escolher um referencial ortonormal $\left\{e_{1}, \ldots, e_{n}\right\}$ tal que $h_{i j}=\lambda_{i} \delta_{i j}$. Reenumerando os vetores da base se necessário, podemos assumir que $\lambda_{n}<0<$ $\lambda_{1} \leq \cdots \leq \lambda_{n-1}$.

Supondo que tal subsequência exista e, por abuso de notação, iremos denotá-la por $\left\{p_{k}\right\}$. Se necessário, consideraremos as subsequências $\left\{\tilde{p_{k}}\right\}$ e $\left\{p_{k}^{\prime}\right\}$, onde $h_{n n j}\left(\tilde{p_{k}}\right) \geq 0$ e $h_{n n j}\left(p_{k}^{\prime}\right)<0$.

Usando a subsequência $\left\{\tilde{p_{k}}\right\}$, temos:

$$
\left|\nabla S\left(\tilde{p_{k}}\right)\right|<\frac{1}{k} \Rightarrow S_{j}\left(\tilde{p_{k}}\right)>-\frac{1}{k} .
$$

Usando que $\lambda_{n}=-\sum_{i}^{n-1} \lambda_{i}$, pois $M$ é maximal, e derivando $S=\sum_{i}^{n} h_{i i}^{2}$ em relação a $j$, podemos escrever $S_{j}\left(\tilde{p_{k}}\right)$ como se segue:

$$
\begin{aligned}
S_{j}\left(\tilde{p_{k}}\right) & =2\left[\sum_{i=1}^{n-1}\left(\lambda_{i} h_{i i j}\right)+\lambda_{n} h_{n n j}\right]\left(\tilde{p_{k}}\right) \\
& =2\left[\sum_{i=1}^{n-1} \lambda_{i} h_{i i j}+\sum_{i=1}^{n-1}\left(-\lambda_{i}\right) h_{n n j}\right]\left(\tilde{p_{k}}\right) \\
& =2 \sum_{i=1}^{n-1} \lambda_{i}\left(h_{i i j}-h_{n n j}\right)\left(\tilde{p_{k}}\right),
\end{aligned}
$$

e portanto

$$
-\frac{1}{2 k}<\sum_{i=1}^{n-1} \lambda_{i}\left(\tilde{p_{k}}\right)\left(h_{i i j}\left(\tilde{p_{k}}\right)-h_{n n j}\left(\tilde{p_{k}}\right)\right) .
$$

Como por hipótese $\left|h_{n n j}\left(\tilde{p_{k}}\right)\right|=\max \left\{\left|h_{i i j}\left(\tilde{p_{k}}\right)\right|, i=1, \ldots, n\right\}$, então $h_{i i j}\left(\tilde{p_{k}}\right)-h_{n n j}\left(\tilde{p_{k}}\right) \leq 0$, pois $h_{n n j}\left(\tilde{p_{k}}\right) \geq 0$. Então

$$
-\frac{1}{2 k}<\sum_{i=1}^{n-1} \lambda_{i}\left(\tilde{p_{k}}\right)\left(h_{i i j}\left(\tilde{p_{k}}\right)-h_{n n j}\left(\tilde{p_{k}}\right)\right) \leq \lambda_{i}\left(\tilde{p_{k}}\right)\left(h_{i i j}\left(\tilde{p_{k}}\right)-h_{n n j}\left(\tilde{p_{k}}\right)\right) \leq 0 .
$$

Tomando o limte com $k \rightarrow \infty$ nos fatores da desigualdade anterior, e usando o Teorema do Confronto, segue que:

$$
\lim _{k \rightarrow \infty} \lambda_{i}\left(\tilde{p_{k}}\right)\left(h_{i i j}\left(\tilde{p_{k}}\right)-h_{n n j}\left(\tilde{p_{k}}\right)\right)=0 .
$$

Mas, por (4.20), podemos tomar subsequência tal que:

$$
0<\lim _{k \rightarrow \infty} \frac{1}{\lambda_{i}\left(\tilde{p_{k}}\right)}=\frac{1}{\tilde{\lambda_{i}}}<+\infty
$$


Portanto, por (4.21) e (4.22) e usando propriedades de limites, temos que:

$$
\begin{aligned}
0 & =\lim _{k \rightarrow \infty} \frac{1}{\lambda_{i}\left(\tilde{p_{k}}\right)} \cdot \lim _{k \rightarrow \infty} \lambda_{i}\left(\tilde{p_{k}}\right)\left(h_{i i j}\left(\tilde{p_{k}}\right)-h_{n n j}\left(\tilde{p_{k}}\right)\right) \\
& =\lim _{k \rightarrow \infty}\left(h_{i i j}\left(\tilde{p_{k}}\right)-h_{n n j}\left(\tilde{p_{k}}\right)\right) .
\end{aligned}
$$

Com isso, como para cada $i$, o limite dessa subtração é nulo, podemos somar um número finito de vezes, que esse limite continuará nulo, e novamente usando que $M^{n}$ é maximal, ou seja,

$$
0=\sum_{i=1}^{n} h_{i i} \Rightarrow 0=\sum_{i=1}^{n} h_{i i j},
$$

e portanto

$$
\sum_{i=1}^{n-1} h_{i i j}=-h_{n n j}
$$

chegamos a conclusão que:

$$
\begin{aligned}
0 & =\sum_{i=1}^{n-1} \lim _{k \rightarrow \infty}\left(h_{i i j}\left(\tilde{p_{k}}\right)-h_{n n j}\left(\tilde{p_{k}}\right)\right) \\
& =\lim _{k \rightarrow \infty}\left(\sum_{i=1}^{n-1} h_{i i j}\left(\tilde{p_{k}}\right)-\sum_{i=1}^{n-1} h_{n n j}\left(\tilde{p_{k}}\right)\right) \\
& =\lim _{k \rightarrow \infty}\left(\sum_{i=1}^{n-1} h_{i i j}\left(\tilde{p_{k}}\right)-(n-1) h_{n n j}\left(\tilde{p_{k}}\right)\right) \\
& =\lim _{k \rightarrow \infty}\left(-h_{n n j}\left(\tilde{p_{k}}\right)-n h_{n n j}\left(\tilde{p_{k}}\right)+h_{n n j}\left(\tilde{p_{k}}\right)\right) \\
& =\lim _{k \rightarrow \infty}\left(-n h_{n n j}\right)\left(\tilde{p_{k}}\right) .
\end{aligned}
$$

Portanto:

$$
\lim _{k \rightarrow \infty} h_{n n j}\left(\tilde{p_{k}}\right)=0 .
$$

Com raciocínio análogo, usando a subsequência $\left\{p_{k}^{\prime}\right\}$, teremos que

$$
\left(h_{i i j}-h_{n n j}\right)\left(p_{k}^{\prime}\right)>0,
$$

então as desigualdades obtidas para a sequencia $\left\{\tilde{p_{k}}\right\}$ serão invertidas, logo:

$$
\sum_{i=1}^{n-1} \lambda_{i}\left(h_{i i j}-h_{n n j}\right)\left(p_{k}^{\prime}\right)=S_{j}\left(p_{k}^{\prime}\right)<\frac{1}{k} .
$$

Como a soma agora é de termos positivos, temos

$$
\begin{gathered}
0 \leq \sum_{i=1}^{n-1} \lambda_{i}\left(h_{i i j}-h_{n n j}\right)\left(p_{k}^{\prime}\right) \leq \lambda_{i}\left(p_{k}^{\prime}\right)\left(h_{i i j}\left(p_{k}^{\prime}\right)-h_{n n j}\left(p_{k}^{\prime}\right)\right)<\frac{1}{k} . \\
\Rightarrow \lim _{k \rightarrow \infty} \lambda_{i}\left(p_{k}^{\prime}\right)\left(h_{i i j}\left(p_{k}^{\prime}\right)-h_{n n j}\left(p_{k}^{\prime}\right)\right)=0 .
\end{gathered}
$$


Analogamente, somando $(n-1)$ termos, distribuindo e comutando os somatórios com o limite, e usando (4.23) chegaremos a conclusão que:

$$
\lim _{k \rightarrow \infty} h_{n n j}\left(p_{k}^{\prime}\right)=0
$$

Então por (4.24) e (4.25), temos que

$$
\lim _{k \rightarrow \infty} h_{n n j}\left(p_{k}\right)=0
$$

Como por hipótese, $\left|h_{n n j}\left(p_{k}\right)\right|=\max \left|h_{i i j}\left(p_{k}\right)\right|, i=1, \ldots, n$, então concluímos que:

$$
\lim _{k \rightarrow \infty} h_{i i j}\left(p_{k}\right)=0, \quad i=1, \ldots, n .
$$

Lema 4.0.7. Seja $M^{n}$ uma hipersuperfície completa e maximal com curvatura de Gauss-Kronecker constante não-nula em $\mathbb{H}_{1}^{n-1}$. Se existir uma subsequência de pontos $\left\{p_{k}\right\}$ definida como em (4.19) e (4.20), tal que:

$$
\left|h_{n-1 n-1 n}\left(p_{k}\right)\right|=\max \left\{\left|h_{i i n}\left(p_{k}\right)\right|, i=1, \ldots, n-1\right\},
$$

então $\lim _{k \rightarrow \infty} h_{i i n}\left(p_{k}\right)=0, \quad i=1, \ldots, n-1$.

Demonstração. Analogamente a prova do lema anterior, suponha que tal subsequência existe e, se necessário, vamos considerar as subsequências $\left\{\tilde{p_{k}}\right\}$ e $\left\{p_{k}^{\prime}\right\}$ tais que $h_{n-1 n-1 n}\left(\tilde{p_{k}}\right) \geq 0$ e $h_{n-1 n-1 n}\left(p_{k}^{\prime}\right)<$ 0 .

Diferenciando $S$ em relação a $n$,

$$
\begin{aligned}
S_{n} & =\left[\sum_{i=1}^{n} h_{i i}^{2}\right]_{n} \\
& =\sum_{i=1}^{n} 2 h_{i i} h_{i i n} \\
& =\sum_{i=1}^{n-2} 2 \lambda_{i} h_{i i n}+2 \lambda_{n-1} h_{n-1 n-1 n}+2 \lambda_{n} h_{n n n},
\end{aligned}
$$

e usando (4.19) temos:

$$
-\frac{1}{k}<S_{n}=\sum_{i=1}^{n-2} \lambda_{i} h_{i i n}+\lambda_{n-1} h_{n-1 n-1 n}+\lambda_{n} h_{n n n} .
$$

Usando que:

$$
h_{n n n}=-\sum_{i=1}^{n-2} h_{i i n}-h_{n-1 n-1 n}, \quad \lambda_{n-1}=-\sum_{i=1}^{n-2} \lambda_{i}-\lambda_{n}
$$

temos que 


$$
\begin{aligned}
S_{n}= & \sum_{i=1}^{n-2} \lambda_{i} h_{i i n}+\lambda_{n-1} h_{n-1 n-1 n}+\lambda_{n} h_{n n n} \\
= & \sum_{i=1}^{n-2} \lambda_{i} h_{i i n}+\left(-\sum_{i=1}^{n-2} \lambda_{i}-\lambda_{n}\right) h_{n-1 n-1 n}+\lambda_{n}\left(-\sum_{i=1}^{n-2} h_{i i n}-h_{n-1 n-1 n}\right) \\
= & \sum_{i=1}^{n-2} h_{i i n}\left(\lambda_{i}-\lambda_{n}\right)-\sum_{i=1}^{n-2} \lambda_{i} h_{n-1 n-1 n}-\lambda_{n} h_{n-1 n-1 n}-\lambda_{n} h_{n-1 n-1 n} \\
& +\left(\sum_{i=1}^{n-2} \lambda_{n} h_{n-1 n-1 n}-\sum_{i=1}^{n-2} \lambda_{n} h_{n-1 n-1 n}\right) \\
= & \sum_{i=1}^{n-2} h_{i i n}\left(\lambda_{i}-\lambda_{n}\right)-\sum_{i=1}^{n-2} h_{n-1 n-1 n}\left(\lambda_{i}-\lambda n\right)-2 \lambda_{n} h_{n-1 n-1 n}-\sum_{i=1}^{n-2} \lambda_{n} h_{n-1 n-1 n} \\
= & \sum_{i=1}^{n-2}\left(\lambda_{i}-\lambda_{n}\right)\left(h_{i i n}-h_{n-1 n-1 n}\right)-n \lambda_{n} h_{n-1 n-1 n} .
\end{aligned}
$$

Agora note que, como a curvatura de Gauss-Kronecker é constante por hipótese, então qualquer derivada direcional da função $F$ se anulará identicamente, então diferenciando $F$ na direção $n$ temos:

$$
\begin{aligned}
0 & =\left(\log \left|\operatorname{det}\left(h_{i j}\right)\right|\right)_{n} \\
& =\sum_{i=1}^{n-2} \frac{1}{\lambda_{i}} h_{i i n}+\frac{1}{\lambda_{n-1}} h_{n-1 n-1 n}+\frac{1}{\lambda_{n}} h_{n n n} \\
& =\sum_{i=1}^{n-2} \frac{1}{\lambda_{i}} h_{i i n}+\frac{1}{\lambda_{n-1}} h_{n-1 n-1 n}+\frac{1}{\lambda_{n}}\left(-\sum_{i=1}^{n-2} h_{i i n}-h_{n-1 n-1 n}\right) \\
& =\sum_{i=1}^{n-2}\left(\frac{1}{\lambda_{i}}-\frac{1}{\lambda_{n}}\right) h_{i i n}+\frac{1}{\lambda_{n-1}} h_{n-1 n-1 n}-\frac{1}{\lambda_{n}} h_{n-1 n-1 n} .
\end{aligned}
$$

Note que

$$
\left(\frac{1}{\lambda_{n-1}}-\frac{1}{\lambda_{n}}\right) h_{n-1 n-1 n}=-\sum_{i=1}^{n-2} \frac{1}{\lambda_{i}} h_{n-1 n-1 n}+\left(\sum_{j=1}^{n} \frac{1}{\lambda_{j}}-\frac{2}{\lambda_{n}}\right) h_{n-1 n-1 n},
$$

logo:

$$
\begin{aligned}
0 & =\sum_{i=1}^{n-2}\left(\frac{1}{\lambda_{i}}-\frac{1}{\lambda_{n}}\right) h_{i i n}-\sum_{i=1}^{n-2} \frac{1}{\lambda_{i}} h_{n-1 n-1 n}+\left(\sum_{j=1}^{n} \frac{1}{\lambda_{j}}-\frac{2}{\lambda_{n}}\right) h_{n-1 n-1 n} \\
& =\sum_{i=1}^{n-2}\left(\frac{1}{\lambda_{i}}-\frac{1}{\lambda_{n}}\right) h_{i i n}-\left(\sum_{i=1}^{n-2} \frac{1}{\lambda_{i}}+\frac{2}{\lambda_{n}}\right) h_{n-1 n-1 n}+\sum_{j=1}^{n} \frac{1}{\lambda_{j}} h_{n-1 n-1 n} .
\end{aligned}
$$


Mas

$$
\begin{aligned}
-\left(\sum_{i=1}^{n-2} \frac{1}{\lambda_{i}}+\frac{2}{\lambda_{n}}\right) & =-\left(\sum_{i=1}^{n-2} \frac{1}{\lambda_{i}}+\frac{2}{\lambda_{n}}+\frac{(n-2)}{\lambda_{n}}-\frac{(n-2)}{\lambda_{n}}\right) \\
& =-\left(\sum_{i=1}^{n-2} \frac{1}{\lambda_{i}}+\frac{n}{\lambda_{n}}-\sum_{i=1}^{n-2} \frac{1}{\lambda_{n}}\right) .
\end{aligned}
$$

Então:

$$
0=\sum_{i=1}^{n-2}\left(\frac{1}{\lambda_{i}}-\frac{1}{\lambda_{n}}\right)\left(h_{i i n}-h_{n-1 n-1 n}\right)+\left(\frac{-n}{\lambda_{n}}+\sum_{j=1}^{n} \frac{1}{\lambda_{j}}\right) h_{n-1 n-1 n}
$$

Multiplicando (4.26) por $\left(\frac{-n}{\lambda_{n}}+\sum_{j=1}^{n} \frac{1}{\lambda_{j}}\right),(4.27)$ por $n \lambda_{n}$, e em seguida somando-as, teremos:

$$
\begin{aligned}
-\left(-\frac{n}{\lambda_{n}}+\sum_{j=1}^{n} \frac{1}{\lambda_{j}}\right) \frac{1}{k}< & \left(-\frac{n}{\lambda_{n}}+\sum_{j=1}^{n} \frac{1}{\lambda_{j}}\right) \sum_{i=1}^{n-2}\left(\lambda_{i}-\lambda_{n}\right)\left(h_{i i n}-h_{n-1 n-1 n}\right) \\
& -\left(-\frac{n}{\lambda_{n}}+\sum_{j=1}^{n} \frac{1}{\lambda_{j}}\right)\left(-n \lambda_{n} h_{n-1 n-1 n}\right) \\
& n \lambda_{n} \sum_{i=1}^{n-2}\left(\frac{\lambda_{n}-\lambda_{i}}{\lambda_{i} \lambda_{n}}\right)\left(h_{i i n}-h_{n-1 n-1 n}\right) \\
& +n \lambda_{n}\left(-\frac{n}{\lambda_{n}}+\sum_{j=1}^{n} \frac{1}{\lambda_{j}}\right) h_{n-1 n-1 n} \\
< & \left(-\frac{n}{\lambda_{n}}+\sum_{j=1}^{n} \frac{1}{\lambda_{j}}\right) \sum_{i=1}^{n-2}\left(\lambda_{i}-\lambda_{n}\right)\left(h_{i i n}-h_{n-1 n-1 n}\right) \\
& +\sum_{i=1}^{n-2}\left(\frac{n}{\lambda_{i}}\right)\left(\lambda_{i}-\lambda_{n}\right)\left(h_{i i n}-h_{n-1 n-1 n}\right) \\
< & \sum_{i=1}^{n-2}\left(-\frac{n}{\lambda_{n}}-\frac{n}{\lambda_{i}}+\sum_{j=1}^{n} \frac{1}{\lambda_{j}}\right)\left(\lambda_{i}-\lambda_{n}\right)\left(h_{i i n}-h_{n-1 n-1 n}\right) .
\end{aligned}
$$

Avaliando em pontos $\left\{\tilde{p_{k}}\right\}$ da subsequência obtida, os dois lados da desigualdade acima, podemos concluir o seguinte:

Como $h_{n-1 n-1 n}\left(\tilde{p_{k}}\right) \geq 0$, então por hipótese segue que

$$
\left(h_{i i n}-h_{n-1 n-1 n}\right)\left(\tilde{p_{k}}\right) \leq 0
$$

Como $\lambda_{n}<0$, então

$$
\lambda_{i}-\lambda_{n}>0
$$


para qualquer ponto de $M^{n}$.

Usando que $\left|\lambda_{n}\right| \geq\left|\lambda_{i}\right|$ para qualquer $i$, e portanto $\frac{1}{\lambda_{n}}+\frac{1}{\lambda_{i}}>0$, teremos a seguinte desigualdade:

$$
-n\left(\frac{1}{\lambda_{n}}+\frac{1}{\lambda_{i}}\right)<0<\frac{1}{\lambda_{n}}+\frac{1}{\lambda_{i}}<\sum_{j=1}^{n} \frac{1}{\lambda_{j}} .
$$

Com isso em mãos, passando o limite para $k \rightarrow \infty$, e usando o teorema do confronto, concluímos que:

$$
\lim _{k \rightarrow \infty}\left(\frac{-n}{\lambda_{n}}-\frac{n}{\lambda_{i}}+\sum_{j=1}^{n} \frac{1}{\lambda_{j}}\right)\left(\lambda_{i}-\lambda_{n}\right)\left(h_{i i n}-h_{n-1 n-1 n}\right)\left(\tilde{p_{k}}\right)=0 .
$$

Como o $\lim _{k \rightarrow \infty} \lambda_{i}\left(\tilde{p_{k}}\right)=\tilde{\lambda_{i}}$, onde $\tilde{\lambda}_{i} \neq 0$, temos que:

$$
\lim _{k \rightarrow \infty}\left(h_{i i n}-h_{n-1 n-1 n}\right)\left(\tilde{p_{k}}\right)=0 .
$$

Sendo assim, usando (4.23) temos

$$
\begin{gathered}
\sum_{i=1}^{n-2} \lim _{k \rightarrow \infty}\left(h_{i i n}-h_{n-1 n-1 n}\right)\left(\tilde{p_{k}}\right)=0, \\
\lim _{k \rightarrow \infty}\left(\sum_{i=1}^{n-2} h_{i i n}-(n-2) h_{n-1 n-1 n}\right)\left(\tilde{p_{k}}\right)=0, \\
\lim _{k \rightarrow \infty}\left(-(n-1) h_{n-1 n-1 n}\left(\tilde{p_{k}}\right)-h_{n n n}\left(\tilde{p_{k}}\right)\right)=0 .
\end{gathered}
$$

Como, por (4.26) temos

$$
\sum_{i=1}^{n-2} \lambda_{i}\left(h_{i i n}-h_{n-1 n-1 n}\right)+\lambda_{n}\left(h_{n n n}-h_{n-1 n-1 n}\right)=S_{n}<\frac{1}{k},
$$

então

$$
0 \leq \lambda_{n}\left(h_{n n n}-h_{n-1 n-1 n}\right)<\frac{1}{k}-\sum_{i=1}^{n-2} \lambda_{i}\left(h_{i i n}-h_{n-1 n-1 n}\right),
$$

e usando que $\lim _{k \rightarrow \infty}\left(h_{i i n}-h_{n-1 n-1 n}\right)\left(\tilde{p_{k}}\right)=0$ e $\lim _{k \rightarrow \infty} \lambda_{n}\left(\tilde{p_{k}}\right)=\tilde{\lambda_{n}}<0$, juntamente com o teorema do confronto, concluímos que

$$
\lim _{k \rightarrow \infty}\left(h_{n n n}\left(\tilde{p_{k}}\right)-h_{n-1 n-1 n}\left(\tilde{p_{k}}\right)\right)=0 .
$$

Com isso em mãos, somando (4.28) e (4.29), temos: 


$$
\begin{aligned}
0 & =\lim _{k \rightarrow \infty}\left(-(n-1) h_{n-1 n-1 n}\left(\tilde{p_{k}}\right)-h_{n n n}\left(\tilde{p_{k}}\right)\right)+\lim _{k \rightarrow \infty}\left(h_{n n n}\left(\tilde{p_{k}}\right)-h_{n-1 n-1 n}\left(\tilde{p_{k}}\right)\right) \\
& =\lim _{k \rightarrow \infty}\left(-n h_{n-1 n-1 n}\left(\tilde{p_{k}}\right)\right) \\
& =\lim _{k \rightarrow \infty} h_{n-1 n-1 n}\left(\tilde{p_{k}}\right)
\end{aligned}
$$

Analogamente, para o caso da subsequência $\left\{p_{k}^{\prime}\right\}$, teremos que:

$$
\lim _{k \rightarrow \infty} h_{n-1 n-1 n}\left(p_{k}^{\prime}\right)=0 .
$$

Sendo assim, concluímos por (4.30) e (4.31) que

$$
\lim _{k \rightarrow \infty} h_{n-1 n-1 n}\left(p_{k}\right)=0 .
$$

Como por hipótese $\left|h_{n-1 n-1 n}\left(p_{k}\right)\right|=\max \left\{\left|h_{i i n}\left(p_{k}\right)\right|, i=1, \ldots, n-1\right\}$ segue que:

$$
\lim _{k \rightarrow \infty} h_{i i n}\left(p_{k}\right)=0, \quad i=1, \ldots, n-1 .
$$

Vamos agora à demonstração do teorema.

Demonstração. (Teorema 4.0.2)

A demonstração segue, essencialmente, como a do teorema anterior, manipulando algebricamente a expressão de $\Delta F$, mas com o objetivo de mostrar agora que inf $S \geq n$. Fixado $p \in M$ consideremos um referencial $\left\{e_{1}, \ldots, e_{n}\right\}$, tal que $h_{i j}=\lambda_{i} \delta_{i j}$ e, reenumerando se necessário a base $\left\{e_{i}\right\}$, podemos assumir que $\lambda_{n}<0<\lambda_{1} \leq \cdots \leq \lambda_{n-1}$.

Como $K$ é constante não-nula, podemos reescrever $\Delta F$, separando os índices $n$ da seguinte forma:

$$
\begin{aligned}
0= & \Delta F \\
= & -\sum_{i} \frac{1}{\lambda_{i}^{2}} h_{i i i}^{2}-\sum_{i \neq j}\left(\frac{1}{\lambda_{i}^{2}}+\frac{2}{\lambda_{i} \lambda_{j}}\right) h_{i i j}^{2}-\sum_{i=1}^{n-1}\left(\frac{1}{\lambda_{i}^{2}}+\frac{2}{\lambda_{i} \lambda_{n}}\right) h_{i i n}^{2} \\
& -\sum_{i=1}^{n-1}\left(\frac{1}{\lambda_{n}^{2}}+\frac{2}{\lambda_{i} \lambda_{n}}\right) h_{n n i}^{2}-\frac{1}{3} \sum_{i \neq j \neq k}\left(\frac{\lambda_{i}+\lambda_{j}+\lambda_{k}}{\lambda_{i} \lambda_{j} \lambda_{k}}\right) h_{i j k}^{2}+n(S-n) .
\end{aligned}
$$

Considerando novamente os subconjuntos $A, B$ e $C$, definidos em (4.16) como no teorema anterior, onde $g_{j} \in\{1,2, \ldots, n\}$ é o maior índice tal que $\left|h_{g_{j} g_{j} j}\right|=\max \left\{\left|h_{i i j}\right|, i=1, \ldots, n\right\}$, podemos reescrever a expressão acima, como: 


$$
\begin{aligned}
0= & -\sum_{i=r+1}^{n} \frac{1}{\lambda_{i i i}^{2}}-\sum_{\substack{i \neq j \\
i, j \leq n-1 \\
(i, j) \neq\left(g_{j}, j\right), j=r+1, \ldots, t}}\left(\frac{1}{\lambda_{i}^{2}}+\frac{2}{\lambda_{i} \lambda_{j}}\right) h_{i i j}^{2}-\sum_{i=1}^{n-1}\left(\frac{1}{\lambda_{i}^{2}}+\frac{2}{\lambda_{i} \lambda_{n}}\right) h_{i i n}^{2} \\
& -\sum_{j=1}^{r}\left[\left(\frac{1}{\lambda_{n}^{2}}+\frac{2}{\lambda_{j} \lambda_{n}}\right) h_{n n j}^{2}+\frac{1}{\lambda_{j}^{2}} h_{j j j}^{2}\right] \\
& -\sum_{j=r+1}^{t}\left[\left(\frac{1}{\lambda_{n}^{2}}+\frac{2}{\lambda_{j} \lambda_{n}}\right) h_{n n j}^{2}+\left(\frac{1}{\lambda_{g_{j}}^{2}}+\frac{2}{\lambda_{j} \lambda_{g_{j}}}\right) h_{g_{j} g_{j} j}^{2}\right] \\
& -\frac{1}{3} \sum_{i \neq j \neq k}\left(\frac{\lambda_{i}+\lambda_{j}+\lambda_{k}}{\lambda_{i} \lambda_{j} \lambda_{k}}\right) h_{i j k}^{2}+n(S-n)-\sum_{j=t+1}^{n-1}\left(\frac{1}{\lambda_{n}^{2}}+\frac{2}{\lambda_{j} \lambda_{n}}\right) h_{n n j}^{2} .
\end{aligned}
$$

Partiremos agora para a análise dos somandos de $\Delta F$.

É fácil ver que, o primeiro e o segundo somando são não positivos. Usando (4.15), concluímos também que o terceiro somando é não-positivo.

Como $h_{j j j}^{2} \geq h_{n n j}^{2}$ para $j+1, \ldots, r$ temos que

$$
\begin{aligned}
\sum_{j=1}^{r}\left[\left(\frac{1}{\lambda_{n}^{2}}+\frac{2}{\lambda_{j} \lambda_{n}}\right) h_{n n j}^{2}+\frac{1}{\lambda_{j}^{2}} h_{j j j}^{2}\right] & \geq \sum_{j=1}^{r}\left(\frac{1}{\lambda_{n}^{2}}+\frac{2}{\lambda_{j} \lambda_{n}}+\frac{1}{\lambda_{j}^{2}}\right) h_{n n j}^{2} \\
& =\sum_{j=1}^{r}\left(\frac{1}{\lambda_{n}}+\frac{1}{\lambda_{j}^{2}}\right)^{2} h_{n n j}^{2} \\
& \geq 0 .
\end{aligned}
$$

Portanto o quarto somando também é não-positivo.

Como $h_{g_{j} g_{j} j}^{2}>h_{n n j}^{2}$ para $j=r+1, \ldots, t$, segue que

$$
\begin{gathered}
\sum_{j=r+1}^{t}\left[\left(\frac{1}{\lambda_{n}^{2}}+\frac{2}{\lambda_{j} \lambda_{n}}\right) h_{n n j}^{2}+\left(\frac{1}{\lambda_{g_{j}}^{2}}+\frac{2}{\lambda_{j} \lambda_{g_{j}}}\right) h_{g_{j} g_{j} j}^{2}\right] \geq \\
\sum_{j=r+1}^{t}\left(\frac{1}{\lambda_{n}^{2}}+\frac{2}{\lambda_{j} \lambda_{n}}+n+\frac{1}{\lambda_{g_{j}}^{2}}+\frac{2}{\lambda_{j} \lambda_{g_{j}}}+n\right) h_{n n j}^{2}= \\
\sum_{j=r+1}^{t}\left[\frac{1}{\lambda_{n}^{2}}+\frac{1}{\lambda_{g_{j}}^{2}}+2 n+\frac{2}{\lambda_{j}}\left(\frac{1}{\lambda_{n}}+\frac{1}{\lambda_{g_{j}}}\right)\right] h_{n n j}^{2} \geq 0 .
\end{gathered}
$$

Assim concluímos que o quinto somando também é não positivo.

Com isso, todos os somandos são não-positivos, talvez com excessão dos termos:

$$
\begin{aligned}
& \sum_{i=1}^{n-1}\left(\frac{1}{\lambda_{i}^{2}}+\frac{2}{\lambda_{i} \lambda_{n}}\right) h_{i i n}^{2}, \\
& \sum_{j=t+1}^{n-1}\left(\frac{1}{\lambda_{n}^{2}}+\frac{2}{\lambda_{j} \lambda_{n}}\right) h_{n n j}^{2} .
\end{aligned}
$$


Sendo assim:

$$
-\sum_{i=1}^{n-1}\left(\frac{1}{\lambda_{i}^{2}}+\frac{2}{\lambda_{i} \lambda_{n}}\right) h_{i i n}^{2}+n(S-n)-\sum_{j=t+1}^{n-1}\left(\frac{1}{\lambda_{n}^{2}}+\frac{2}{\lambda_{j} \lambda_{n}}\right) h_{n n j}^{2} \geq 0 .
$$

Pois $\Delta F=0$ e os outros somandos são não-positivos.

Para analisarmos a soma acima, faremos o uso do Lema 3.0.4, assim, obtemos uma sequência de pontos $\left\{p_{k}\right\}$ satisfazendo:

$$
S\left(p_{k}\right)<\inf S+\frac{1}{k}, \quad\left|\nabla S\left(p_{k}\right)\right|<\frac{1}{k}, \quad \Delta S\left(p_{k}\right)>-\frac{1}{k},
$$

e se necessário, $\lim _{k \rightarrow \infty} \lambda_{i}\left(p_{k}\right)=\tilde{\lambda}_{i}, \tilde{\lambda}_{i} \neq 0$, já que $\lambda_{i}$ aparece no denominador das frações.

Agora, fixando $i=n$ e $j=t+1, \ldots, n-1$, de acordo com o Lema 4.0.6 temos $\lim _{k \rightarrow \infty} h_{n n j}\left(p_{k}\right)=0$, já que $C=\{t+1, \ldots, n-1\}$, o que implica $\left|h_{n n j}\right|=\max \left\{\left|h_{i i j}\right|, i=1, \ldots, n\right\}$.

Note que, para $1 \leq i \leq n-2$, usando o fato de $M$ ser maximal, temos:

$$
\begin{aligned}
\frac{1}{\lambda_{i}^{2}}+\frac{2}{\lambda_{i} \lambda_{n}} & =\frac{\lambda_{n}+2 \lambda_{i}}{\lambda_{i}^{2} \lambda_{n}} \\
& =\frac{-\sum_{j=1, j \neq i}^{n-2} \lambda_{j}-\lambda_{i}-\lambda_{n-1}+2 \lambda_{i}}{\lambda_{i}^{2} \lambda_{n}} \\
& =\frac{\left(\lambda_{i}-\lambda_{n-1}\right)-\sum_{j=1, j \neq i}^{n-2} \lambda_{j}}{\lambda_{i}^{2} \lambda_{n}}>0 .
\end{aligned}
$$

Assim, exceto por

$$
\left(\frac{1}{\lambda_{n-1}^{2}}+\frac{2}{\lambda_{n-1} \lambda_{n}}\right) h_{n-1 n-1 n}^{2}=\frac{\lambda_{n-1}-\sum_{j=1}^{n-2} \lambda_{j}}{\lambda_{n-1}^{2} \lambda_{n}} h_{n-1 n-1 n}^{2},
$$

que não podemos afirmar nada sobre o sinal do numerador, todos os outros termos de (4.32) são não-negativos.

Para tratar desse caso específico, a estratégia é de conseguirmos uma estimativa para $h_{n-1 n-1 n}$. Então se existir uma sequência de pontos $\left\{p_{k}\right\}$ tal que $\left|h_{n-1 n-1 n}\left(p_{k}\right)\right|=\max \left\{\left|h_{i i n}\left(p_{k}\right)\right|, i=\right.$ $1, \ldots, n-1\}$, , então pelo Lema 4.0.7 temos:

$$
\lim _{k \rightarrow \infty} h_{i i n}\left(p_{k}\right)=0, \quad i=1,2, \ldots, n-1,
$$

e o problema seria trivialmente resolvido.

Sendo assim, excluindo os finitos pontos $\left\{p_{k}\right\}$ tal que $\left|h_{n-1 n-1 n}\left(p_{k}\right)\right|=\max \left\{\left|h_{i i n}\left(p_{k}\right)\right|, i=1, \ldots, n-\right.$ $1\}$, nós obtemos uma sequencia $\left\{p_{k}\right\}$ satisfazendo $\left|h_{l l n}\left(p_{k}\right)\right|=\max \left\{\left|h_{i i n}\left(p_{k}\right)\right|, i=1, \ldots, n-1\right\}$, com $l \neq n-1$. 
Portanto:

$$
\begin{aligned}
& \left(\frac{1}{\lambda_{l}^{2}}+\frac{2}{\lambda_{l} \lambda_{n}}\right) h_{l l n}^{2}+\left(\frac{1}{\lambda_{n-1}^{2}}+\frac{2}{\lambda_{n-1} \lambda_{n}}\right) h_{n-1 n-1 n}^{2} \geq \\
& {\left[\frac{1}{\lambda_{l}^{2}}+\frac{1}{\lambda_{n-1}^{2}}+2\left(\frac{1}{\lambda_{l} \lambda_{n}}+\frac{1}{\lambda_{n-1} \lambda_{n}}\right)\right] h_{n-1 n-1 n}^{2}=} \\
& {\left[\left(\frac{1}{\lambda_{l}}-\frac{1}{\lambda_{n-1}}\right)+2\left(\frac{\lambda_{n}+\lambda_{l}+\lambda_{n-1}}{\lambda_{l} \lambda_{n-1} \lambda_{n}}\right)\right] h_{n-1 n-1 n}^{2} \geq 0 .}
\end{aligned}
$$

Avaliando $\left[-\sum_{i=1}^{n-1}\left(\frac{1}{\lambda_{i}^{2}}+\frac{2}{\lambda_{i} \lambda_{n}}\right) h_{i i n}^{2}+n(S-n)-\sum_{j=t+1}^{n-1}\left(\frac{1}{\lambda_{n}^{2}}+\frac{2}{\lambda_{j} \lambda_{n}}\right) h_{n n j}^{2}\right]\left(p_{k}\right)$, teremos que, como $\Delta F=0$, e todos os outros termos são não-positivos, obrigatoriamente, $n(S-n)\left(p_{k}\right) \geq 0$, logo, com $k \rightarrow \infty$, do Lema 3.0.4 temos

$$
n(\inf S-n) \geq 0 \Rightarrow \inf S \geq n \text {. }
$$

Logo, por 3.0.2, $0 \leq S \leq n \Rightarrow \sup S \leq n$.

$$
\begin{gathered}
\Rightarrow n \leq \inf S \leq \sup S \leq n . \\
\Rightarrow S=n .
\end{gathered}
$$

Sendo assim, novamente pelo Teorema 3.0.3, $M^{n}$ é isométrico ao cilindro hiperbólico $\mathbb{H}^{1}\left(c_{1}\right) \times$ $\mathbb{H}^{n-1}\left(c_{2}\right)$.

Segue do Teorema 4.0.2 o seguinte resultado:

Corolario 4.0.8. Seja $M^{4}$ uma hipersuperfície maximal completa em $\mathbb{H}_{1}^{5}(-1)$ com curvatura de Gauss-Kronecker constante negativa, então $M^{4}$ é isométrica ao cilindro hiperbólico $\mathbb{H}^{1}\left(c_{1}\right) \times \mathbb{H}^{3}\left(c_{2}\right)$.

Demonstração. Seguindo a argumentação do Corolário 4.0.5, note que, como $K$ é por hipótese, constante negativa, e $K$ é o produto das curvaturas principais de $M^{4}$, então, obrigatoriamente 3 curvaturas principais possuem o mesmo sinal. Portanto, $M^{4}$ tem $4-1$ curvaturas principais com o mesmo sinal. Com isso, todas as hipóteses do Teorema 4.0.2 estão satisfeitas e o resultado segue direto. 


\section{Referências Bibliográficas}

[1] K. Akutagawa. On spacelike hypersurfaces with constant mean curvature in the de sitter space. Mathematische Zeitschrift, 196(1):13-19, 1987. 1

[2] A. Barros and P. Andrade. Introdução à geometria projetiva. Textos Universitários. Rio de Janeiro: Editora SBM, 2010. 3

[3] E. Bombieri, E. De Giorgi, and E. Giusti. Minimal cones and the Bernstein problem. Invent. Math., 7:243-268, 1969. 1

[4] A. Brasil, A. G. Colares, O. Palmas, et al. Complete spacelike hypersurfaces with constant mean curvature in the de sitter space: A gap theorem. Illinois Journal of Mathematics, 47(3):847-866, 2003. 1

[5] M. Brozos-Vázquez, E. García-Río, P. Gilkey, S. Nikčević, and R. Vázquez-Lorenzo. The geometry of walker manifolds. Synthesis Lectures on Mathematics and Statistics, 4, 2009.

[6] E. Calabi. Examples of bernstein problems for some nonlinear equations, 1970 global analysis (proc. sympos. pure math., vol. xv, berkeley, calif., 1968) pp. 223-230 amer. Math. Soc., Providence, RI. 1

[7] L. Cao and G. Wei. A new characterization of hyperbolic cylinder in anti-de sitter space. Journal of mathematical analysis and applications, 329(1):408-414, 2007. 1

[8] R. Chaves, L. Sousa, and B. Valério. New characterizations for hyperbolic cylinders in anti-de sitter spaces. Journal of Mathematical Analysis and Applications, 393(1):166-176, 2012. 2

[9] S.-Y. Cheng and S.-T. Yau. Maximal space-like hypersurfaces in the lorentz-minkowski spaces. Annals of Mathematics, pages 407-419, 1976. 1

[10] S. d. Chern, M. Do Carmo, and S. Kobayashi. Minimal submanifolds of a sphere with second fundamental form of constant length. In Manfredo P. do Carmo-Selected Papers, pages 47-63. Springer, 2012. 1

[11] H. F. de Lima, F. R. dos Santos, and M. A. L. Velásquez. New characterizations of hyperbolic cylinders in semi-riemannian space forms. Journal of Mathematical Analysis and Applications, 434(1):765-779, 2016.

[12] M. P. do Carmo. O método do referencial móvel. Instituto de Matemática Pura e Aplicada, 1976.

[13] M. P. Do Carmo. Formas diferenciais e aplicacİões. Number 37. Instituto de Matemática pura e aplicada, 1983.

[14] M. P. do Carmo. Geometria riemanniana. Instituto de Matemática Pura e Aplicada, 1988. $10,18,23$ 
[15] A. Goddard. Some remarks on the existence of spacelike hypersurfaces of constant mean curvature. In Mathematical Proceedings of the Cambridge Philosophical Society, volume 82, pages 489-495. Cambridge Univ Press, 1977. 1

[16] J. N. Gomes, H. F. de Lima, F. R. dos Santos, and M. A. L. Velásquez. On the complete linear weingarten spacelike hypersurfaces with two distinct principal curvatures in lorentzian space forms. Journal of Mathematical Analysis and Applications, 418(1):248-263, 2014.

[17] T. Ishihara et al. Maximal spacelike submanifolds of a pseudo-riemannian space of constant curvature. The Michigan mathematical journal, 35(3):345-352, 1988. iii, v, 1, 2, 24, 35, 36, 39, 42

[18] J. Lee. Introduction to Smooth Manifolds, volume 218. Springer Science \& Business Media, 2012.

[19] S. Montiel. An integral inequality for compact spacelike hypersurfaces in de sitter space and applications to the case of constant mean curvature. Indiana University Mathematics Journal, 37(4):909-917, 1988. 1

[20] S. Morita. Geometry of differential forms, volume 201. American Mathematical Soc., 2001. 11

[21] A. C. Muniz Neto. Topicos de Geometria Diferencial. SBM, 2014. 5

[22] H. Omori. Isometric immersions of riemannian manifolds. Journal of the Mathematical Society of Japan, 19(2):205-214, 1967. 36

[23] C.-K. Peng and C.-L. Terng. Minimal hypersurfaces of spheres with constant scalar curvature. Annals of Math. Studies, (103):177-198, 2016. 43

[24] O. Perdomo. New examples of maximal space like surfaces in the anti-de sitter space. Journal of Mathematical Analysis and Applications, 353(1):403-409, 2009. 2

[25] F. W. Warner. Foundations of differentiable manifolds and Lie groups, volume 94. Springer Science \& Business Media, 2013.

[26] B. Wu. On complete spacelike hypersurfaces with constant m-th mean curvature in an anti-de sitter space. International Journal of Mathematics, 21(05):551-569, 2010. 2

[27] S.-T. Yau. Harmonic functions on complete riemannian manifolds. Communications on Pure and Applied Mathematics, 28(2):201-228, 1975. 2, 35

[28] Y. Zhu. Ricci curvature of spacelike hypersurfaces in de sitter space. Differential Geometry and its Applications, 31(4):517-523, 2013. 


\section{Índice Remissivo}

Cilindro hiperbólico, 2

Conexão, 5

Curvatura, 6

de Ricci, 7

escalar, 7

formas de, 13

média, 7

média de uma hipersuperfície, 18

seccional, 7

tensor, 7

Diferencial covariante, 18

Distribuição, 10

completamente integrável, 11

involuta, 11

paralela, 11

Equação

de Codazzi, 18

de Gauss, 17

Fibrado vetorial, 5

Lema de Cartan, 17

Referencial móvel, 13

Segunda forma fundamental, 9, 18

Subfibrado vetorial, 5

Teorema

de Frobenius, 11

de Levi-Civita, 6

Variedade

diferenciável, 4

integral, 11

topológica, 4 